\title{
Rough Isometries of Order Lattices
} and Groups

\author{
Dissertation \\ zur Erlangung des mathematisch-naturwissenschaftlichen Doktorgrades \\ "Doctor rerum naturalium" \\ der Georg-August-Universität Göttingen
}

vorgelegt von

Andreas Lochmann

aus Bad Pyrmont

Göttingen 2009 
Referenten der Dissertation:

Prof. Dr. Thomas Schick

Prof. Laurent Bartholdi, Ph.D.

Mitglieder der Prüfungskommission:

Prof. Dr. Thomas Schick

Prof. Laurent Bartholdi, Ph.D.

Prof. Dr. Rainer Kress

Prof. Dr. Ralf Meyer

Prof. Dr. Ulrich Stuhler

Prof. Dr. Andreas Thom

Tag der mündlichen Prüfung:

6. August 2009 
Gewidmet

meinen Eltern 



\title{
Preface
}

\begin{abstract}
A One of the distances is 143, the distance between the other pair of points is 141, and all other distances are 99. You know that your error in measurement is not greater than 1 . Is this result then compatible with the hypothesis, that the four points constitute a square in Euclidean space?

Another example: You distribute five posts equidistantly along straight rails, then watch a train driving on the rails and take the times at which it passes the posts. The times you measure are: 0, 100, 202, 301, 401. However, the time the clocks show might deviate by up to 1 from the clock at the starting post. Are the measured times consistent with the assumption that the train has constant speed in Newtonian mechanics?

These two puzzles are metaphors for a general problem in the application of mathematics. We have to use measurements that might contain errors to verify predictions of an ideal theory. If, for example, you find a function $f$ between vector spaces to fulfill linearity only approximately, i.e. there is $\epsilon>0$ such that

$$
d(f(x+y), f(x)+f(y)) \leq \epsilon
$$

for all $x, y$; can you find a perfectly linear function $g$ which is near $f$ ? Ulam stated this question during a talk around 1941, and Hyers found a positive solution shortly after by applying scaling arguments $([\mathrm{Hy}])$. Ulam and Hyers continued their collaboration, and were able to give positive answer in $\mathrm{HU}$ ] to the following question in 1947: Is every $\epsilon$-isometric homomorphism of realvalued continuous function spaces in bounded distance to an isometry?
\end{abstract}

Theorem 1 (Hyers-Ulam) 1

Let $K, K^{\prime}$ be compact metric spaces and let $E$ and $E^{\prime}$ be the spaces of all real valued continuous functions on $K$ and $K^{\prime}$, respectively. Let $T(f)$ be a homomorphism of $E$ onto $E^{\prime}$ which is also an $\epsilon$-isometry for some arbitrary, fixed $\epsilon \geq 0$. Then there exists an isometric transformation $U(f)$ of $E$ onto $E^{\prime}$ such that $\|U(f)-T(f)\| \leq 21 \epsilon$ for all $f$ in $E$. In particular, the underlying metric spaces $K$ and $K^{\prime}$ are homeomorphic.

This theorem evoked several similar results for linear functions ([Ra2]), but also about stability of differential equations, the stability of group actions (GKM]), and approximate group homomorphisms, as defined by Ulam in [U], section VI.1; see [HR] for a survey on this topic. 
It is interesting to see that the isometry of the function spaces implies conditions of their underlying metric spaces - in this case they are homeomorphic. Our main interest during a large part of this thesis will be function spaces of Lipschitz functions, and their stability against rough isometries.

Rough isometries are in some sense the prototype of approximative structure: the description of Hyers' $\epsilon$-linear functions would not be possible without a metric on the vector spaces. The idea simply is to replace an identity $x=y$ by $d(x, y) \leq \epsilon$. In this sense, each description of approximations needs metric spaces. As the natural mappings of metric spaces are Lipschitz maps, isometries, and isometric embeddings, their rough counterparts are the most natural approximative tools, among them the rough isometry (or $\epsilon$-isometry) as measure to describe the similarity of metric spaces. Our goal is to shed some light on the interplay of rough isometries with order lattice and group structures, but in particular to demonstrate their use in the study of the geometry of Lipschitz function spaces.

There are detailed books and various articles on many aspects of Lipschitz function spaces, including isometries between them. A nice survey is Weaver's book on Lipschitz Algebras Wv (cf. Section 2.6, where Weaver elaborates on the exact same questions we want to tackle here, but in a non-coarse context and under different conditions). However, to the knowledge of the author, no book nor article dealt with their coarse geometry yet. On the other hand, Lipschitz functions naturally appear in many aspects of coarse geometry, such as the Levy concentration phenomenon or the definition of Lipschitz-Hausdorff distance in Gv2. But they are not dealt with as metric spaces either.

The structure of this thesis is as follows: We first give an overview of basic notions in handling with metric spaces and order lattices. We assume that the reader is familiar with metric spaces, so this part will just cover our use of infinities in metric spaces, the definition of hyperconvex and injective metric spaces, and those notions from coarse geometry which apply to our situation.

The first chapter deals with a generalization of Birkhoff's metric lattices to put the supremum metric on Lipschitz function spaces into a common context. During this chapter we derive several useful tools for to handle distances in Lipschitz function spaces, and introduce several metrics for Lipschitz functions, familiar ones as well as some more exotic distance functions. The final section in this chapter will define a very important notion for our analysis, a version of irreducibility which depends on the chosen metric of a lattice. We further see some first, general properties of this new kind of irreducibility.

The second chapter will concentrate on Lipschitz function spaces with supremum metric, and we will examine the question, whether they are roughly isometric when their underlying spaces are. We first take a look at a simple, but efficient smoothening algorithm, then take a digression to demonstrate a corresponding Lemma for hyperconvex-space-valued Lipschitz functions.

We then revive the situation of the first chapter, and define a rough version of isomorphism for intervaluation metric lattices, which fits well into the context of our new irreducibility-condition. By introducing minimal Lipschitz functions (which we call " $\Lambda$-functions"), we provide a lattice-theoretic base of the Lipschitz function space (see Definition [76), and put the smoothening re- 
sult of the first section into this more advanced context, re-proving it using new techniques:

Theorem 2 2

Let $X, Y$ be metric $c_{+}$spaces, $\epsilon \geq 0$. For each $\epsilon$-isometry $\eta: X \rightarrow Y$, there is a $4 \epsilon$-ml-isomorphism $\kappa: \operatorname{Lip} Y \rightarrow \operatorname{Lip} X$ such that $\kappa$ is $\epsilon$-near $f \mapsto f \circ \eta$ for all $f \in \operatorname{Lip} Y$ (see Definitions [6, 29, 80, 89.).

We then continue to analyze our $\Lambda$-functions and find them to be exactly the irreducible elements of the first chapter. This allows us to state a converse of Theorem 2

Theorem 3 3

Let $X, Y$ be complete metric $c_{+}$spaces and $\epsilon \geq 0$. For each $\epsilon$-ml-isomorphism $\kappa: \operatorname{Lip} Y \rightarrow \operatorname{Lip} X$ there is an $88 \epsilon$-isometry $\eta: X \rightarrow Y$, such that $\kappa$ is $62 \epsilon$-near $f \mapsto f \circ \eta$ for all $f \in \operatorname{Lip} Y$.

As we make use of the order-lattice structure of the Lipschitz function spaces in our proofs, the following theorem by Kaplansky $\mathrm{Kp}$ is related to our results as well and we state it in its formulation by Birkhoff ([Bi1, 2nd ed. p. 175f.):

Theorem 4 (Kaplansky) 4 Any compact Hausdorff space is (up to homeomorphism) determined by the lattice of its continuous functions.

However, the proofs we present here not only make use of the lattice structure of Lip $X$, but of a metric on it as well. In this sense, the comparison with Kaplansky's Theorem 4 is not perfect.

We then ask whether it is possible to state Theorems 2 and 3 in a more general context, and give counter-examples to a broad range of different metrics on Lipschitz function spaces, among them the seemingly convenient $L^{1}$-metric. We close this chapter with an example concerning quasi-isometries, and an application in the theory of scaling limits.

The third chapter discusses the use of rough isometries in the context of finitely generated groups. One cannot overvalue the importance of coarse methods in Geometric Group Theory, which is in large parts based on the notion of quasi-isometry. We begin this chapter with another digression, demonstrating how some proofs can be reformulated in a rough context, while this is not possible with others. We then try to point out the possibilities rough isometries add to Geometric Group Theory, by providing a specifically rough isometry invariant (the exponential growth rate), and then show the connection between symmetries in some virtually abelian groups and rough isometries between them. We then continue with a theorem motivated by Bartholdi and translate it into the rough context in the final section, where we show that uniformly rough and quasi-isometries imply commensurability.

Finally a remark to our notation: $\mathbb{N}_{0}$ denotes the non-negative integers, $\mathbb{N}^{*}$ the positive integers, $C_{n}$ denotes the cyclic group of order $n$. We sometimes drop function brackets where feasible. 
Acknowledgements. The author wants to thank the "Graduiertenkolleg Gruppen und Geometrie" for supporting his research financially, through conferences and workshops, and by supporting the great open atmosphere at the institute. Best thanks go to Prof. Dr. Thomas Schick, Prof. Laurent Bartholdi, and Prof. Dr. Andreas Thom for their friendly support and great expertise, and to Prof. Dr. Themistocles Rassias for pointing the author at important works in connected fields. The author thanks his family and friends for always supporting him through the course of the doctorate, and in particular Dr. Johannes Härtel for being friend, critic, and guide.

Danksagung. Der Autor möchte dem "Graduiertenkolleg Gruppen und Geometrie" dafür danken, dass es seine Forschung sowohl finanziell als auch durch Konferenzen und Workshops und durch den Erhalt der großartigen Atmosphäre am Institut unterstützt hat. Bester Dank geht an Prof. Dr. Thomas Schick, Prof. Laurent Bartholdi, Ph.D., und Prof. Dr. Andreas Thom für ihre freundliche Unterstützung und große Expertise, sowie an Prof. Dr. Themistocles Rassias für die Verweise auf wichtige Arbeiten in verwandten Gebieten. Der Autor dankt seiner Familie und seinen Freunden für die stete Unterstützung im Laufe des Doktorats, und insbesondere Dr. Johannes Härtel für Freundschaft, Kritik und Anleitung.

Georg-August-Universität Göttingen, Germany, 2009

eMail lochmann@uni-math.gwdg.de 


\section{Contents}

\begin{tabular}{lll}
\hline 0 & Basic Notions & 11
\end{tabular}

0.1 Basic Notions in Metric Spaces . . . . . . . . . . . . . . . . 11

$0.1 .1 \quad$ Infinite Metrics . . . . . . . . . . . . . . . . . . 11

0.1 .2 Iniective and Hvperconvex Metric Spaces . . . . . . . . 13

0.1.3 Basic Notions of Coarse Geometrv . . . . . . . . . . . . 16

0.2 Basic Notions in Order Lattices . . . . . . . . . . . . . . . 17

1 Order Lattices with Metrics 23

1.1 Valuation Metric Lattices . . . . . . . . . . . . . . . . . 23

1.2 Ultravaluation Metrics . . . . . . . . . . . . . . . . . . . . . 32

1.3 Intervaluation Metrics . . . . . . . . . . . . . . . . . . . . 34

1.4 Complete Metric/Lattice-Irreducibility . . . . . . . . . . . . . . . 39

2 Rough Isometries of Lipschitz Function Spaces 43

2.1 Smoothening of Lipschitz Functions . . . . . . . . . . . . . . . 43

2.1.1 Hyperconvex and Non-Hvperconvex Codomains . . . . . . 48

2.1.2 Algorithmic Aspects . . . . . . . . . . . . . . . . . . 51

2.2 Rough ml-Isomorphisms . . . . . . . . . . . . . . . . . . . 51

$2.3 \quad \Lambda$-Functions . . . . . . . . . . . . . . . . . . 54

$2.4 \Lambda$-Functions are Completely ml-Irreducible . . . . . . . . . . . 59

2.5 Inducing Rough Isometries . . . . . . . . . . . . . . . . . . 61

2.6 Other Metrics for $\operatorname{Lip} X \ldots \ldots \ldots \ldots$

2.7 Quasi-Isometries . . . . . . . . . . . . . . . . . . . 67

2.8 Scaling limits $\ldots \ldots \ldots \ldots$

\begin{tabular}{|lll}
\hline 3 & Rough Isometries of Groups & $\mathbf{7 1}$
\end{tabular}

3.1 The Theorem of Mazur-Ulam . . . . . . . . . . . . . . . 71

3.1.1 Rough Abelianness . . . . . . . . . . . . . . . . . 75

3.2 Coarse Relations for Groups . . . . . . . . . . . . . . . 75

3.3 Exponential Growth Rate . . . . . . . . . . . . . . 77

3.4 The Abelian Case . . . . . . . . . . . . . . . . . . . . . . . . . . . . 78

3.5 Rough Isometries of Quotients with Finite Kernel . . . . . . . . . 81

3.6 Shared Isometries . . . . . . . . . . . . . . . . . . . 82

3.7 Shared Rough and Quasi-Isometries . . . . . . . . . . . . 87

\begin{tabular}{lr}
\hline Bibliographv & 95
\end{tabular} 


\section{Chapter 0}

\section{Basic Notions}

\subsection{Basic Notions in Metric Spaces}

\subsubsection{Infinite Metrics}

As we will deal with lattice-theoretic notions, it is convenient to ensure the existence of a greatest possible distance in a metric space. To do so without having to restrict to bounded metric spaces, we will extend our notion of metric spaces to include infinite distances.

Definition 5 5

We define the binary operation + on $[0, \infty]$ to be as expected on $[0, \infty)$, and set $\infty+z=z+\infty=\infty$ as well as $z \leq \infty$ for all $z \in[0, \infty]$. We call $z \in[0, \infty]$ positive if $z \neq 0$.

Definition 6

$A$ pseudo-metric + space $(X, d)$ is a non-empty set $X$ together with a mapping $d: X \times X \rightarrow[0, \infty]$ which has the following properties:

1. $d(x, x)=0$ for all $x \in X$, and

2. $d(x, z) \leq d(x, y)+d(y, z)$ for all $x, y z \in X$.

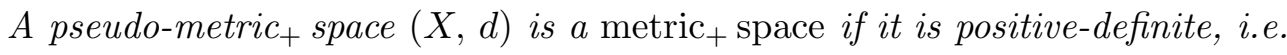

3. $d(x, y)=0$ implies $x=y$ for all $x, y \in X$.

The mapping $d$ is then called a "metric", "metric + ", or "distance".

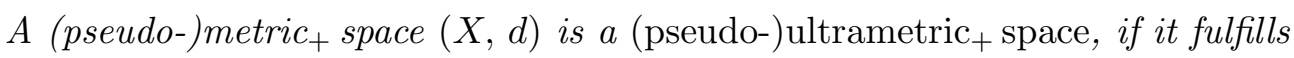

1. $d(x, z) \leq d(x, y) \vee d(y, z)$ for all $x, y z \in X$, 
where $\vee$ denotes the maximum.

$A$ (pseudo-)metric + space $(X, d)$ is called a true (pseudo-)metric space if $d(x, y)$ $\neq \infty$ for all $x, y \in X$. This equals the common notion of a metric space in the literature, like $[B B I]$.

Definition 7

The diameter $\operatorname{diam} A$ of a non-empty subset $A$ in a pseudo-metric + space is the supremum of all distances of pairs of points in $A$.

$A$ bounded (pseudo-)metric space is a (pseudo-)metric + space $X$ with finite diameter $\operatorname{diam} X<\infty$.

Definition 8 8

$A n$ isometry [isometric embedding] is a bijection [injection] between pseudo-metric $c_{+}$spaces which preserves the distance.

In most cases we call metrics just " $d$ " without reference to the metric space, as it should be clear from the elements which metric is meant.

We will make heavy use of the symbol

$$
\left|d\left(x, x^{\prime}\right)-d\left(y, y^{\prime}\right)\right| \leq z
$$

for some $x, x^{\prime}, y, y^{\prime}$ in $X$ or $Y$ and $z \in[0, \infty]$. To make sense of this in case one of the distances becomes infinite, we define the former symbol to be equivalent to

$$
d\left(x, x^{\prime}\right) \leq d\left(y, y^{\prime}\right)+z \quad \text { and } \quad d\left(y, y^{\prime}\right) \leq d\left(x, x^{\prime}\right)+z .
$$

In particular, we find $|\infty-\infty|=0$. This might seem unfamiliar. Note however, that $\left|z-z^{\prime}\right|$ can be perfectly understood as a metric + on $[0, \infty]$ itself.

Remark There is no non-trivial convergence to $\infty$ in this metric, $\infty$ is just an infinitely far away point. We will not need a convergence to infinity anyway; the addition of $\infty$ serves to obtain completeness of order lattices, particularly of $[0, \infty]$ itself.

Furthermore, it is obvious that a metric + space $X$ always is a disjoint union of true metric spaces $X_{j}$ with $d\left(x, x^{\prime}\right)=\infty$ if and only if $x \in X_{j}, x^{\prime} \in X_{k}$ with $j \neq k$, for $j, k \in J$. We call the $X_{j}$ components of $X$. We call $X$ complete, if all of its components are complete as true metric spaces, i.e. each Cauchy sequence converges.

The difference between metric + and true metric spaces is rather small, as a metric + $_{+}$space merely is a collection of true metric spaces. For example, the topology of a metric + space is the disjoint union of the topology of its components, and any converging sequence eventually is contained in one of these components, with only finitely many exceptions at the beginning of the sequence. 


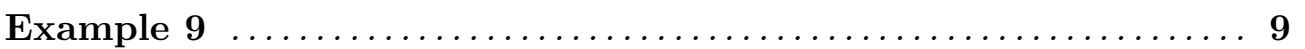

The algebra of continuous functions $f: \mathbb{R} \rightarrow \mathbb{R}$ together with the supremum metric

$$
d_{\infty}(x, y):=\sup _{x \in \mathbb{R}}|f(x)-f(y)|
$$

is a metric $c_{+}$space. Its components are those functions with finite distance to each other, so the subset of bounded functions constitutes the component of the zero function, and the identity belongs to another component.

Example 10

Similar to the usual definition of a norm on a vector space, it is possible to define

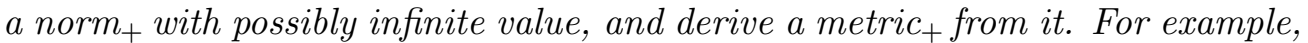
the true metric space of square-summable complex sequences is a component of the metric space of all complex sequences with norm $_{+}$

$$
\left\|\left(x_{1}, x_{2}, \ldots\right)\right\|_{2}:=\sqrt{\sum_{j \in \mathbb{N}^{*}}\left|x_{j}\right|^{2}} \in[0, \infty]
$$

for any complex sequence $\left(x_{1}, x_{2}, \ldots\right)$.

Definition 11

The Hausdorff-distance of two subsets $A$ and $B$ in a pseudo-metric + space is the infimum of all non-negative numbers $r$ such that for each $x \in A$ there is $y \in B$ with $d(x, y) \leq r$ and vice versa.

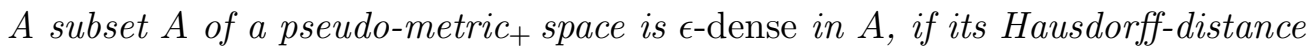
to $A$ is less than or equals $\epsilon$. A dense subset is a 0-dense subset. A roughly dense subset is a subset for which its Hausdorff-distance to $A$ is finite.

Definition 12

A pseudo-metric + space $X$ is separable if it contains a countable dense subset.

\subsubsection{Injective and Hyperconvex Metric Spaces}

Definition 13 ...................................... 13

A short map (sometimes called a "metric map") between pseudo-metric $c_{+}$spaces $X$ and $Y$ is a 1-Lipschitz map $\pi: X \rightarrow Y$, this means it fulfills $d_{Y}(\pi x, \pi y) \leq$ $d_{X}(x, y)$ for all $x, y \in X$.

Short maps are the canonical morphisms to construct a category Met of true metric spaces, as a bijective short map whose inverse is short as well is an isometry. They form morphisms in the category Met $_{+}$of metric spaces as well, as short maps map componentwise.

Definition 14 ...................................... 14

An injective metric ${ }_{+}$space $X$ is an injective object in the category $\mathbf{M e t}_{+}$, i.e. 
for each metric space $Y$ and short map $f: Y \rightarrow X$, and each short embedding $i: Y \hookrightarrow Z$ of $Y$ into another metric + space $Z$, there is a short map $g: Z \rightarrow X$ which extends $f(f=g \circ i)$.

Proposition 15

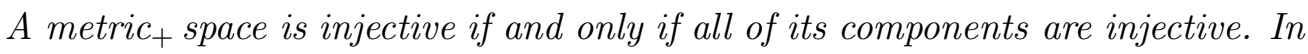
particular, a true metric space is injective in Met if and only if it is injective in Met $_{+}$.

Proof Note that a short map maps a component into a single component of the codomain, i.e. the map which assigns each metric + space its set of components is a functor.

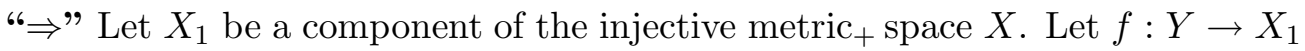

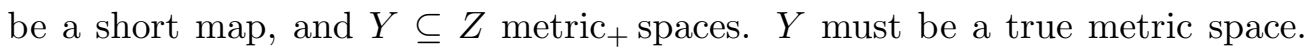
Let $Z_{1}$ be the component of $Y$ in $Z$. Extend $\left.f\right|_{Z_{1}}$ to the whole of $X$, its image must still be in the same component $X_{1}$. Now choose an arbitrary point $x_{0} \in X_{1}$ and map all remaining components of $Z$ to this point. This yields an extension $g: Z \rightarrow X_{1}$ of $f$.

" $\Leftarrow$ " Let $Y \subseteq Z, f: Y \rightarrow X$, where $X$ is componentwise injective. Choose a point $x_{0} \in X$. Extend $f$ componentwise on each component of $Z$ which intersects $Y$; map each remaining component of $Z$ to $x_{0}$.

As next, we state the classical result that metric spaces are injective if and only if they are hyperconvex. This accounts for true metric spaces as well as for

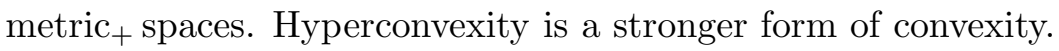

Definition 16

Let $X$ be a metric space. $X$ is convex in the sense of Menger ([Me], p. 81) if for each $x, y \in X$ there is $z \in X \backslash\{x, y\}$ such that $d(x, y)=d(x, z)+d(z, y)$.

$X$ is totally convex (or convex in the sense of $[E K]$ ) if for all $a, b \in(0, \infty]$ with $d(x, y) \leq a+b$ there is $z \in X$ with $d(x, z) \leq a$ and $d(z, y) \leq b$.

$X$ is hyperconvex if for any family of points $\left(x_{j}\right)_{j \in J} \subseteq X$ and numbers $r_{j} \in[0, \infty]$ with $d\left(x_{j}, x_{k}\right) \leq r_{j}+r_{k}$ for all $j \in J$, with $J$ an arbitrary index set, there is an element $z \in X$ with $d\left(x_{j}, z\right) \leq r_{j}$ for all $j \in J$. In simpler words: If any family of balls could theoretically intersect pairwise (given their radii), they all intersect.

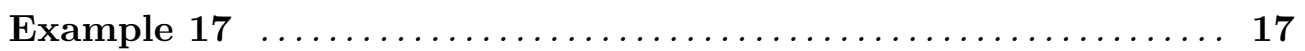

Typical examples for hyperconvex true metric spaces are (real) trees and $\mathbb{R}^{n}$ with $L^{\infty}$-metric as well as certain subsets thereof. In particular, $\mathbb{R}$ and each real interval are hyperconvex.

Proposition 18

A metric space $X$ is hyperconvex if and only if all of its components are hyperconvex. 
Proof All $x_{j}$ with infinite $r_{j}$ can be neglected. On the other hand, if two points $x_{j}, x_{k} \in X$ are in different components, at least of one $r_{j}$ or $r_{k}$ must be infinite, hence the hyperconvexity property always reduces to hold on a single component of $X$. On the other hand, assume $X$ is hyperconvex. Given a family of balls with center $x_{j}$ and radius $r_{j}$ in one component $X_{1} \subseteq X$, we may use hyperconvexity in $X$ to gain the desired element $z \in X$. As long as at least two of the $r_{j}$ were finite, we must choose $z \in X_{1}$. If only one $r_{j}$ is finite, we may choose $z=x_{j}$.

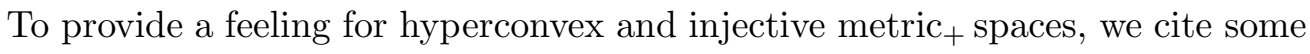
assorted theorems without giving proofs. Due to Propositions 15 and 18, there are no obstructions to generalize the original statements to metric + spaces.

Theorem 19 ([AP], Theorem 2.4) 19

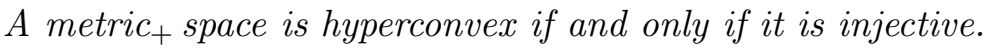

Theorem 20 ([EK], Theorem 3.1) 20

A product of arbitrarily many hyperconvex metric + spaces with supremum metric ${ }_{+}$is hyperconvex. (This statement is much easier in the $\mathbf{M e t}_{+}$form than the original statement for true metric spaces.)

Theorem 21 ([EK], Proposition 3.2)

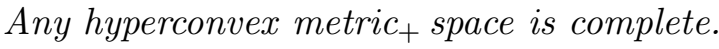

Theorem 22 (Baillon's Theorem; [EK], Theorem 5.1)

The intersection of a descending chain of non-empty hyperconvex subsets of a bounded metric space is non-empty and hyperconvex.

Theorem 23 ([EK], Theorem 6.1) 23

The fixed point set of a short map $f: X \rightarrow X$ acting on a hyperconvex bounded metric space $X$ is non-empty and hyperconvex.

Theorem 24 ([EK], Theorem 9.6) 24

Let $X$ be hyperconvex, $K>0$. The family of all bounded $K$-Lipschitz functions $X \rightarrow X$ with supremum metric is hyperconvex.

Theorem 25 ([I] $]$, Theorem 2.1)

Each true metric space $X$ has an injective envelope (i.e. a minimal injective space into which $X$ isometrically embeds), which is unique up to isometry.

Theorem 26 ([I] , Remark 2.11) 26

The injective envelope of a compact space is compact.

Theorem 27 ([AP], Theorem 3.3)

If $X$ is a metric space, $A \subseteq X$ hyperconvex, $B \subseteq X$ such that $A$ and $B$ have identical closures in $X$, then $B$ is hyperconvex as well. 
Theorem 28 ([AP], Theorem 3.9) 28

Let $X$ be hyperconvex. Then $A \subseteq X$ is hyperconvex if and only if it is a retract of $X$ by a contracting retraction.

\subsubsection{Basic Notions of Coarse Geometry}

A well written introduction to coarse geometry is the book by Burago, Burago and Ivanov $([\mathrm{BBI}])$. In the following, let $X$ and $Y$ be metric + spaces.

Definition 29

Two (set theoretic) mappings $\alpha, \beta: X \rightarrow Y$ are $\epsilon$-near to each other, $\epsilon \geq 0$, if $d_{Y}(\alpha x, \beta x) \leq \epsilon \forall x \in X$. (We drop brackets where feasible.)

$A$ (set theoretic) mapping $\alpha: X \rightarrow Y$ is $\epsilon$-surjective, $\epsilon \geq 0$, if for each $y \in Y$ there is $x \in X$ such that $d_{Y}(\alpha x, y) \leq \epsilon$.

Definition 30

$A$ (not neccessarily continuous) map $\eta: X \rightarrow Y$ is called a $(\lambda, \epsilon)$-quasiisometric embedding, $\epsilon, \lambda \geq 0$ (which shall always imply $\lambda, \epsilon \in \mathbb{R}$ ), if

$$
\lambda^{-1} d_{X}\left(x, x^{\prime}\right)-\epsilon \leq d_{Y}\left(\eta x, \eta x^{\prime}\right) \leq \lambda d_{X}\left(x, x^{\prime}\right)+\epsilon
$$

for all $x, x^{\prime} \in X$.

A pair $\eta: X \rightarrow Y, \eta^{\prime}: Y \rightarrow X$ of $(\lambda, \epsilon)$-quasi-isometric embeddings is called a $(\lambda, \epsilon)$-quasi-isometry if $\eta \circ \eta^{\prime}$ and $\eta^{\prime} \circ \eta$ are $\epsilon$-near the identities on $Y$ and $X$, respectively. When we speak of a "quasi-isometry $\eta: X \rightarrow Y$ " a corresponding map $\eta^{\prime}$ shall always be implied.

$X$ and $Y$ are called quasi-isometric, if there is a quasi-isometry between them.

Definition 31

$A(1, \epsilon)$-quasi-isometric embedding, $\epsilon \geq 0$, is called a $\epsilon$-isometric embedding. It fulfills

$$
\left|d_{X}\left(x, x^{\prime}\right)-d_{Y}\left(\eta x, \eta x^{\prime}\right)\right| \leq \epsilon
$$

for all $x, x^{\prime} \in X$.

An $\epsilon$-isometry is a $(1, \epsilon)$-quasi-isometry. The map $\eta: X \rightarrow Y$ is called a rough isometry if there is some $\epsilon \geq 0$ such that $\eta$ is an $\epsilon$-isometry.

$X$ and $Y$ are called $\epsilon$-isometric [roughly isometric], if there is an $\epsilon$-isometry [any $\epsilon$-isometry] between them.

Historical Remark It is difficult to attribute the concept of rough isometry to a single person, as it was always present in the notion of quasi-isometry, which itself was an obvious generalization of what was then called pseudo-isometry by Mostow in his 1973-paper about rigidity (see [Mo, Gv1, Kn]). Recent developments about the stability of rough isometries can be found in [Ra1]. 


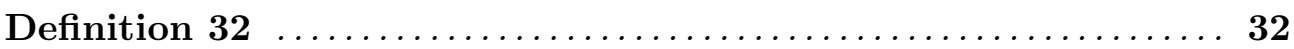

A mapping $f: X \rightarrow Y$ is called $(K, \epsilon)$-Lipschitz (i.e. "K-Lipschitz map on $\epsilon$-scale" in [Gv3]), $\epsilon, K \geq 0$, iff

$$
d_{Y}(f(x), f(y)) \leq K \cdot d_{X}(x, y)+\epsilon \quad \forall x, y \in X .
$$

If $\epsilon=0, f$ is $K$-Lipschitz (continuous). Define $\operatorname{Lip}_{K, \epsilon}(X, Y)$ to be the set of all $(K, \epsilon)$-Lipschitz functions $X \rightarrow Y$, and $\operatorname{Lip}_{K, \epsilon} X:=\operatorname{Lip}_{K, \epsilon}(X,[0, \infty])$, $\operatorname{Lip} X:=\operatorname{Lip}_{1,0}(X)$.

If nothing else is said, $[0, \infty]=\mathbb{R}_{\geq 0} \cup\{\infty\}$ is the default codomain for a Lipschitz function.

Assume $f$ to be a $(K, \epsilon)$-Lipschitz function on $X$ and $f(x)=\infty$ for some $x \in X$. Then clearly $f(y)=\infty$ for all $y$ in finite distance to $x$. Thus, if $X$ is a true metric space, we have $\operatorname{Lip} X=\operatorname{Lip}\left(X, \mathbb{R}_{\geq 0}\right) \cup\{\infty\}$.

\subsection{Basic Notions in Order Lattices}

Good starting points for Lattice Theory are Grätzer $(\underline{\mathrm{Gr}})$ and the classical book by Birkhoff ([Bi1]).

Definition 33 33

$A$ lattice $(L, \wedge, \vee)$ is a set $L$ together with two mappings $\wedge, \vee: L \times L \rightarrow L$ which are commutative, associative and fulfill the absorption laws $f \wedge(f \vee g)=$ $f \vee(f \wedge g)=f$ for all $f, g \in L$. A lattice is a partially ordered set by

$$
f \leq g \quad: \Leftrightarrow f \wedge g=f \stackrel{\text { by absorption }}{\Leftrightarrow} \quad f \vee g=g
$$

$L$ is distributive if $\vee$ and $\wedge$ distribute over each other.

$L$ is bounded (as a lattice) if there exists a smallest element $0 \in L$ and a largest element $1 \in L$.

$L$ is complete if all infima and all suprema of all subsets of $L$ exist in $L$. (A complete lattice always is bounded.)

$L$ is complemented if $L$ is bounded and for each $f \in L$ there is a complement $g \in L$ such that $f \vee g=1$ and $f \wedge g=0$.

$L$ is a Boolean lattice if it is distributive and complemented.

Example 34

Let $X$ be some set, and let $L$ be any family of subsets of $X$ closed under $\cap$ and $\cap$. Then $(L, \cap, \cup)$ is a distributive lattice, sometimes called a "ring of sets" (we will stick to "lattice of sets"). If for all $A \in L$ the complement $X \backslash A \in L$ as well, then $(L, \cap, \cup)$ is a Boolean lattice ("field of sets"). 
Example 35 ....................................... 35

Let $X$ be a topological space, and $T$ the family of all open sets in $X . T$ is a lattice by union and intersection, bounded by $\emptyset$ and $X$, distributive (as it is a lattice of sets). However, if $X$ is Hausdorff, then $T$ is complete if and only if $T$ is the discrete topology, and if and only if $T$ is complemented.

Definition 36

Let $(L, \wedge, \vee)$ be a lattice.

An element $p \in L$ is join-irreducible if, whenever $p=f \vee g$ with $f, g \in L$, then $p=f$ or $p=g$.

An element $p \in L$ is join-prime if, whenever $p \leq f \vee g$ with $f, g \in L$, then $p \leq f$ or $p \leq g$.

An element $p \in L$ is completely join-irreducible if, whenever $p=\bigvee_{j \in J} f_{j}$, with $f_{j} \in L$, then $p=f_{j}$ for some $j \in J, J$ an arbitrary index set. Same for completely join-prime.

$A$ lower set in a partially ordered set $P$ is a subset $Q$ of $P$ with: $f \leq g, g \in Q$, $f \in P$ implies $f \in Q$.

$A$ sublattice of $L$ is a subset of $L$ closed under $\wedge$ and $\vee$.

An ideal is a sublattice $I \subseteq L$ such that $f \wedge g \in I$ whenever $f \in L$ and $g \in I$ (equivalent definition: a sublattice which is a lower set).

$A$ proper ideal is an ideal I which is a proper subset of $L$.

A principal ideal is an ideal $I$ which is generated by a single element.

$A$ prime ideal is a proper ideal $P$ of a Boolean algebra for which holds: If $f, g \in L$ and $f \wedge g \in P$, then $f \in P$ or $g \in P$. The family of all prime ideals in $L$ is called $P(L)$.

Proposition $37 \quad \ldots . \ldots \ldots \ldots \ldots \ldots \ldots \ldots \ldots \ldots \ldots \ldots \ldots \ldots . \ldots \ldots$

In a distributive lattice, join-irreducibility and join-primeness are equivalent ([Bi1], Lemma III.3.1).

Proof Let $p \in L$ be join-prime. Then it is join-irreducible by definition. Now let $p \in L$ be join-irreducible and $p \leq f \vee g$ with $f, g \in L$. We find

$$
p=p \wedge(f \vee g)=(p \wedge f) \vee(p \wedge g) .
$$

As $p$ is join-irreducible, we have $p=p \wedge f$, this is $p \leq f$, or $p=p \wedge g$, which means $p \leq g$.

Example 38

We note that a prime ideal is not the same as a principal ideal generated by a join-prime element:

The positive integers equipped with least common divisor and greatest common multiple constitute a distributive and unbounded lattice $L=\left(\mathbb{N}^{*}, \mathrm{gcd}, \mathrm{lcm}\right)$ 
with 1 as least element. Join-irreducible/join-prime elements are exactly the prime powers. The subset $A=\{5,15,50,150\}$ is an example for a sublattice in L. The least ideal encompassing $A$ is the subset of all divisors of 150 . It is a principal ideal generated by 150 . The principal ideal which is generated by a join-irreducible element $p^{n}$, p prime, is just $\left\{1, p, p^{2}, \ldots, p^{n}\right\}$. The subset $B$ of all powers of 7 is a non-principal proper ideal. It is not a prime ideal: $\operatorname{gcd}(14,35)=7$, but neither $14 \in B$ nor $35 \in B$. The subset $C=\mathbb{Z} \backslash 7 \mathbb{Z}$ of all positive integers except the multiples of 7 is a prime ideal: If $7 \nmid \operatorname{gcd}(f, g)$, then $7 \nmid f$ or $7 \nmid g$. On the other hand, the subset $\mathbb{Z} \backslash 6 \mathbb{Z}$ is not even an ideal.

Example 39 ..................................... 39

Each ideal in a lattice is a lower set, but not vice versa: In the lattice $L=$ $\left(\mathbb{N}^{*}, \operatorname{gcd}, 1 \mathrm{~cm}\right)$ the subset $Q:=\{1,5,25,29\}$ is a lower set, but not an ideal, because $\operatorname{lcm}(5,29)=145 \notin Q$.

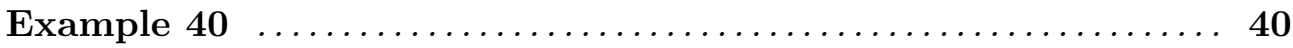

Let $X$ be any set of at least two elements, and consider its powerset $(L=$ $\wp(X), \cap, \cup)$. Its join-irreducibles are the one-element subsets and the empty set. A principal ideal generated by $A \subseteq X$ is $\wp(A) \subseteq \wp(X)$. Given any $x \in X$ consider $\mathcal{F}_{x}:=\{A \subseteq X: x \notin A\} . \mathcal{F}_{x}$ is a prime ideal.

In Lipschitz function spaces, principal ideals which are generated by a joinirreducible are more interesting, as join-irreducibles tend to interact in more interesting ways: Their intersections can be non-trivial.

Theorem 41 41

(Representation Theorem for Distributive Lattices,

G. Birkhoff 1933, M. H. Stone 1936; $[\mathbf{G r}]$, Th. II.1.19)

A lattice is distributive if and only if it is isomorphic to a lattice of sets.

Proof Birkhoff proved a very similar representation theorem for finite distributive lattices in Bi1] (see next Theorem), Stone later extended the proof to infinite distributive lattices as well. In addition, he showed that a complemented distributive lattice is isomorphic to a complemented lattice of sets. The main idea of the proof is to show that the map

$$
\begin{aligned}
\pi: L & \rightarrow \wp(P(L)) \\
f & \mapsto\{p \in P(L): f \notin p\}
\end{aligned}
$$

between $L$ and the power set of the set $P(L)$ of all prime ideals in $L$ is an injective homomorphism, and hence $\left.\pi\right|_{\operatorname{im} \pi}$ constitutes an isomorphism between $L$ and a sublattice of $\wp(P(L))$.

Subsequently, each distributive lattice is isomorphic to a sublattice of a powerset. Today there is a broad variety of representation theorems for (distributive) lattices, particularly as lattices of open, closed or clopen subsets in topological 
spaces. These theorems are subsumed under the term "Stone-type dualities". An earlier version is the following theorem by Birkhoff for finite distributive lattices. We will not make use of it, but its main idea of reconstructing a distributive lattice solely from its join-irreducibles will be a major theme in Chapter 2

Theorem 42 (Birkhoff's Representation Theorem) 42

Let $L$ be any finite distributive lattice, and $J \subseteq L$ the subset of join-irreducibles. $J$ is a partially ordered set (not a sublattice!). The family of lower sets in $J$ is a lattice of sets, and isomorphic to L ([Bi1], Corollary III.3.2).

Of particular interest for us is $L=\operatorname{Lip} X$ for some metric + space $X$, with $\wedge$ and $\checkmark$ pointwise minimum and maximum respectively, and $\Lambda, \vee$ pointwise infimum and supremum. Lip $X$ is a distributive lattice, as the distributivity is inherited from $(\mathbb{R}, \wedge, \vee)$. The following proposition is a special case of Lemma 6.3 in $[\mathrm{He}$ and Proposition 1.5.5 in $\mathrm{Wv}$.

Proposition 43

Let $X$ be a metric space. Then $\operatorname{Lip} X$ is complete as a lattice.

Proof Let $J$ be some arbitrary index set, and let $f_{j}$ be in $\operatorname{Lip} X$ for each $j \in J$. Obviously, $[0, \infty]$ is complete as a lattice, with $\bigwedge_{\emptyset}=\infty$ and $\bigvee_{\emptyset}=0$. So we define pointwise

$$
g(x):=\bigvee_{j \in J} f_{j}(x), \quad h(x):=\bigwedge_{j \in J} f_{j}(x)
$$

and observe that $g$ and $h: X \rightarrow Z$ are Lipschitz: For arbitrary $x, y \in X$ holds

$$
h(x) \leq f_{j}(x) \leq f_{j}(y)+d(x, y)
$$

for all $j \in J$, and thus, by passing to the infimum:

$$
h(x) \leq h(y)+d(x, y) .
$$

Same for $g$.

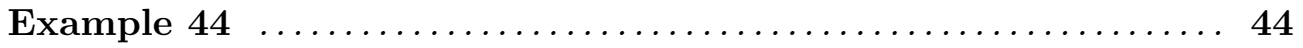
The space $C([0,1],[0,1])$ of real-valued continuous functions $[0,1] \rightarrow[0,1]$ is not a complete lattice with pointwise minimum and maximum: Choose $f_{j}(x)=$ $0 \vee(1-j \cdot x), j \in \mathbb{N}^{*}$, the infimum is not continuous. The same example shows that the space $\bigcup_{K \geq 0} \operatorname{Lip}_{K, 0} X$ of all Lipschitz-functions with arbitrary Lipschitz constant is not a complete lattice.

Example 45

Besides its Stone representation, we want to provide another, more intuitive 
representation of $\operatorname{Lip} X$ by a lattice of sets, using its hypograph (cp. "epigraph" in [Ro]]

$$
\begin{aligned}
\text { hyp : } \operatorname{Lip} X & \rightarrow \wp(X \times[0, \infty]) \\
f & \mapsto\{(x, r): f(x) \leq r\} .
\end{aligned}
$$

(im hyp, $\cap, \cup)$ obviously is isomorphic to $(\operatorname{Lip} X, \wedge, \vee)$ as a lattice; however, they are not yet isomorphic as complete lattices: Infinite unions of the closed sets in im hyp are not closed in general - we have to use the union with closure " $\cup$ " instead of the traditional union. (Alternatively, we could identify subsets of $X \times[0, \infty]$ with the same closure.) 


\section{Chapter 1}

\section{Order Lattices with Metrics}

\subsection{Valuation Metric Lattices}

For the most part of this thesis, we will consider the supremum metric +

$$
d_{\infty}(f, g):=\bigvee_{x \in X}|f(x)-g(x)|
$$

on $\operatorname{Lip} X$. But before we come to further investigate this, we present Birkhoff's Bi1 definition of a metric lattice and demonstrate the difference to our situation.

Definition 46

$A$ valuation on a lattice $L$ is a function $v: L \rightarrow \mathbb{R}$ which satisfies the modular law

$$
v(f)+v(g)=v(f \wedge g)+v(f \vee g) \quad \forall f, g \in L
$$

$A$ valuation $v$ on $L$ is called isotone [positive] if for all $f, g \in L$ the relation $f<g$ implies $v(f) \leq v(g)[v(f)<v(g)]$.

If $L$ is totally ordered, then each function $v: L \rightarrow \mathbb{R}$ is a valuation. It is isotone [positive] if and only if $v$ is [strictly] monotonically increasing.

Example 47

Let $L=\left(\mathbb{N}^{*}, \operatorname{gcd}, \mathrm{lcm}\right)$. Then each logarithm is a positive valuation on $L$.

Example 48

Let $V$ be any finite dimensional vector space, and $L=\mathrm{PG}(V)$ its lattice of subvector spaces, with $\wedge$ the intersection and $\vee$ the span (the projective geometry of $V)$. Then the dimension function is a positive valuation on $L$.

Historical Remark The theory of valuations has been mainly developed and popularized by John von Neumann and Garrett Birkhoff. In the early years of 
the 1930s, von Neumann worked on a variation of the ergodic hypothesis, and inadvertently competed with George David Birkhoff. Only some years later, his son Garrett Birkhoff pointed von Neumann at the use of lattice theory in Hilbert spaces. He wrote about this in a note of the Bulletin of the AMS in 1958 [Bi2].

John von Neumann's brilliant mind blazed over lattice theory like a meteor, during a brief period centering around 1935-1937. With the aim of interesting him in lattices, I had called his attention, in 19331934, to the fact that the sublattice generated by three subspaces of Hilbert space (or any other vector space) contained 28 subspaces in general, to the analogy between dimension and measure, and to the characterization of projective geometries as irreducible, finitedimensional, complemented modular lattices.

As soon as the relevance of lattices to linear manifolds in Hilbert space was pointed out, he began to consider how he could use lattices to classify the factors of operator-algebras. One can get some impression of the initial impact of lattice concepts on his thinking about this classification problem by reading the introduction of [...], in which a systematic lattice-theoretic classification of the different possibilities was initiated. [...]

However, von Neumann was not content with considering lattice theory from the point of view of such applications alone. With his keen sense for axiomatics, he quickly also made a series of fundamental contributions to pure lattice theory.

The modular law in its earliest form (as dimension function) appears in two papers from 1936 by Glivenko and von Neumann (Gl], $\underline{\mathrm{vN}}$ ). Von Neumann used it (and lattice theory in general) in his paper to define and study Continuous Geometry (aka. "pointless geometry"), and later applied his knowledge to found Quantum Logic in his Mathematical Foundations of Quantum Mechanics. A later survey about metric posets is $\underline{M n}$.

Example 49

Let $(X, \Sigma, \mu)$ be a probability space. The $\sigma$-algebra $\Sigma$ is a Boolean lattice by union and intersection. Let $c \in \mathbb{R}$ be arbitrary, then

$$
v(A):=\mu(A)+c
$$

defines an isotone valuation on $\Sigma$ with $v(\emptyset)=c$. The valuation $v$ is positive if and only if there are no null sets in $X$ other than $\emptyset$.

Proof: Let $A \neq \emptyset$ be a null set. Then $\emptyset \subsetneq A$, but $\mu(\emptyset)=0=\mu(A)$. Conversely, if $A \subsetneq B \in \Sigma$, and $v(A)=v(B)$, then define $C:=B \backslash A$. B is a disjoint union of $A$ and $C$, so by $\sigma$-additivity of $\mu$ holds $\mu(A)=\mu(B)=\mu(A)+\mu(C)$, hence $\mu(C)=0$. 
The distance function $d_{v}(A, B):=v(A \cup B)-v(A \cap B)$ (whose properties will be proved in Lemma (53) is the measure of the symmetric difference $A \triangle B$ of $A$ and $B$, if $A \triangle B \in \Sigma$. It relates to the Hausdorff distance just as the 1-distance of functions relates to the supremum distance.

We further exploit the connection between $d_{v}$ and the symmetric difference. The symmetric difference as an operation makes sense only in complemented lattices (power sets are examples for complemented lattices). But although all distributive lattices can be represented by a lattice of sets, and hence can be embedded into a complemented lattice, they need not be complemented by themself.

Example 50 50

Let $L=(\mathbb{Z}, \min , \max )$. A possible representation of $L$ is the family

$$
A(L):=\{(-\infty, n] \cap \mathbb{Z} \mid n \in \mathbb{Z}\}
$$

of subsets of $\mathbb{Z}$, equipped with union and intersection. This lattice is orderisomorphic to $L$. The canonical distance in $\mathbb{Z}$ is given by the L-valuation $v(n):=n$, as well as by the counting measure of the symmetric difference of sets in $A(L)$. However, $L$ is distributive, but not complemented, there is no probability measure $\mu$ on $A(L)$ which corresponds to $v$, and the symmetric difference of two sets in $A(L)$ does not yield a set of $A(L)$ again; these problems are all connected to each other, as they all base on the fact that $L$ is not complemented. They still persist even if one completes $L$ to $L^{\prime}:=(\mathbb{Z} \cup\{ \pm \infty\}, \min , \max )$ and generalizes $v$ to a valuation with infinity.

Note that the existence of symmetric differences $f \Delta g$, of complements $f^{c}$, and of difference sets $f \backslash g$ are equivalent to each other in complete lattices of sets:

$$
\begin{aligned}
f^{c} & :=1 \Delta f=1 \backslash f \quad \text { with } \quad 1:=\bigvee_{f \in L} f \\
f \Delta g & :=(f \wedge g)^{c} \wedge(f \vee g)=(f \backslash g) \cup(g \backslash f) \\
f \backslash g & :=f \wedge g^{c}=f \Delta(f \wedge g)
\end{aligned}
$$

In particular, complement, symmetric difference, and difference are unique.

Lip $X$ never is a complemented lattice. Hence, there is no difference defined on Lip $X$; however, given a valuation $v$ and $f, g \in \operatorname{Lip} X$, we may define a difference valuation of $f$ and $g$ by:

$$
w(f, g):=v(f)-v(f \wedge g) .
$$

As $f \wedge g \leq f$, we conclude that for positive or isotone $v$, the difference valuation $w$ always is non-negative.

Proposition 51 (Difference valuation properties)

Let $v$ be an isotone valuation on a distributive lattice $L$ and $w$ its difference valuation. Then the following holds for all $f, g, h \in L$ : 
1. $w(f, g)+w(g, f)=v(f \vee g)-v(f \wedge g)=: d_{v}(f, g)$

2. $w(f, g)=w(f, g \vee h)+w(f \wedge h, g) \quad$ (cut law)

3. $f \leq g \Rightarrow w(f, g)=0$

4. $v$ is positive if and only if for all $f, g \in L$ holds: $w(f, g)=0 \Leftrightarrow f \leq g$.

5. Let $w: L \times L \rightarrow \mathbb{R}$ be a map satisfying property (2), $c \in \mathbb{R}$ arbitrary, and let $0 \in L$ be a least element. Then $v(f):=w(f, 0)+c$ is a valuation with $w$ as difference valuation, and all valuations with $w$ as difference valuation are of this form. If $w(f, g) \geq 0$ for all $f, g \in L$, then $v$ is isotone.

Proof (1) Simply by the defining property of $v$ :

$$
\begin{aligned}
w(f, g)+w(g, f) & =v(f)-2 v(f \wedge g)+v(g) \\
& =v(f \vee g)-v(f \wedge g)
\end{aligned}
$$

(2) By the defining property of $v$ we know:

$$
\begin{aligned}
v(f \wedge h)+v(f \wedge g) & =v((f \wedge g) \vee(f \wedge h))+v((f \wedge g) \wedge(f \wedge h)) \\
& =v(f \wedge(g \vee h))+v(f \wedge g \wedge h)
\end{aligned}
$$

Inserting this into the definition of $w$ yields:

$$
\begin{aligned}
w(f, g) & =v(f)-v(f \wedge g) \\
& =v(f)-v(f \wedge(g \vee h))+v(f \wedge h)-v(f \wedge g \wedge h) \\
& =w(f, g \vee h)+w(f \wedge h, g)
\end{aligned}
$$

We call this equation "cut law" in view of its meaning in Venn diagrams (see Figure 1.11).

(3) Using (2): $f=f \wedge g \Rightarrow w(f, g)=w(f \wedge g, g)+w(f, g \vee g)=2 w(f, g)$.

$(4$, " $\Rightarrow ") f \leq g$ is equivalent to $f \wedge g=f$, and by positivity, equivalent to $v(f \wedge g)=v(f)$.

$(4, " \Leftarrow ")$ Let $f<g$. Then $f=g \wedge f$, and

$$
v(g)-v(f)=v(g)-v(g \wedge f)=w(g, f) .
$$

By isotony we know $v(f) \leq v(g)$, assume $v(f)=v(g)$, then $w(g, f)=0$, and hence $g \leq f$, contradicting $f<g$.

(5) First of all we deduce an easy consequence of properties (2) and (3):

$$
w(f \vee g, g)=w((f \vee g) \wedge f, g)+w(f \vee g, f \vee g)=w(f, g)
$$

With this at hand, we can easily conclude that $v$ is a valuation and that $w$ is its difference valuation:

$$
\begin{aligned}
v(f) & =w(f, 0)+c=w(f \wedge g, 0)+w(f, g)+c \\
& =v(f \wedge g)+w(f, g) \\
\text { and } \quad v(f \vee g) & =w(f \vee g, 0)+c=w(g, 0)+w(f \vee g, g)+c \\
& =v(g)+w(f, g) \\
\Rightarrow \quad v(f)+v(g) & =v(f \vee g)+v(f \wedge g)
\end{aligned}
$$




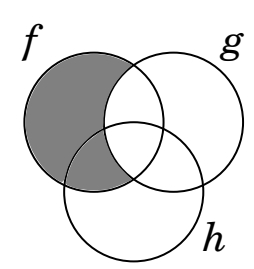

$w(f, g)$

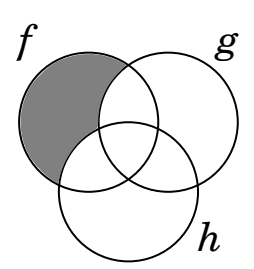

$=w(f, g \vee h)$

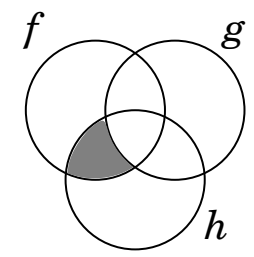

$w(f \wedge h, g)$

Figure 1.1: Visualization of the cut law of difference valuations (Proposition [51 2), using Venn diagrams. Using a representation $\pi$, the set $\pi(f) \backslash \pi(g)$ is cut along $\pi(h)$ to give $\pi(f) \backslash \pi(g \vee h)$ and $\pi(f \wedge h) \backslash \pi(g)$.

Let $w(f, g) \geq 0, f \leq g$, then $v(g)-v(f)=v(g)-v(f \wedge g) \geq 0$, i.e. $v$ is isotone. Now let $v$ be any valuation with $w$ as difference valuation, then

$$
v(f)=v(f)-v(f \wedge 0)+v(0)=w(f, 0)+v(0)
$$

obviously holds, choose $c=v(0)$.

Proposition 51,5 shows the equivalence of the concepts of valuation and difference valuation for complete lattices, so we may define the term "difference valuation" without reference to an actual valuation:

Definition 52 52

$A$ difference valuation on a distributive lattice $L$ is a function $w: L \times L \rightarrow \mathbb{R}$ which satisfies the cut law

$$
w(f, g)=w(f, g \vee h)+w(f \wedge h, g) .
$$

A difference valuation $w$ is called isotone if its values are non-negative, and positive, if $w(f, g)=0$ implies $f \leq g$.

The following Lemma is a part of Theorem X.1 and a note in subsection X.2 of Bi1, and can equally well be stated in terms of valuations as well as difference valuations:

Lemma 53

Let $v$ be an isotone valuation on the distributive lattice $L$. Then

$$
d_{v}(f, g):=v(f \vee g)-v(f \wedge g)
$$

defines a pseudo-metric with the following properties:

1. If there is a least element $0 \in L$, then

$$
v(f)=v(0)+d_{v}(f, 0) \quad \text { for all } \quad f \in L,
$$


$d(f, g)$

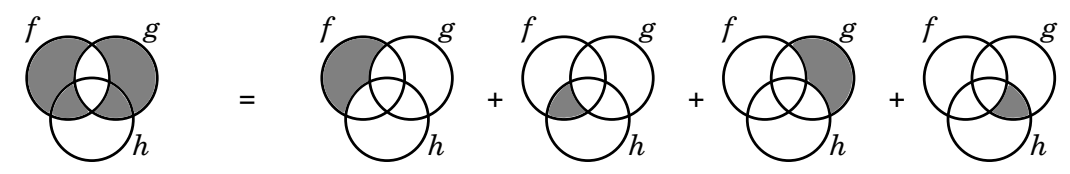

$\wedge$

$\Lambda$

$\Lambda$

$\wedge$

$d(f, h)$
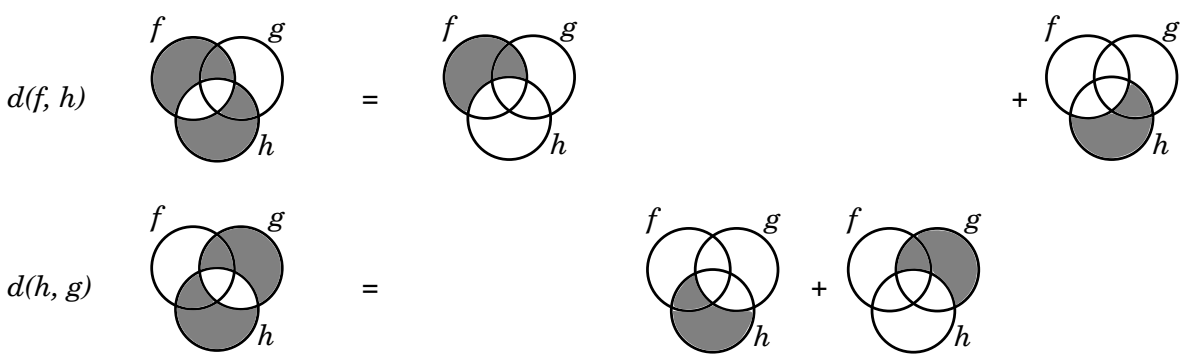

Figure 1.2: Proof of the triangle inequality for valuation metric lattices, e.g. of Lipschitz function spaces with $L^{1}$-metric. Note that $f, g, h$ are functions in this case, and symbolized by sets via Stone duality.

\section{2. $d_{v}$ is a metric if and only if $v$ is positive.}

We call $d_{v}$ a valuation (pseudo-)metric. A lattice together with a valuation metric is sometimes called a metric lattice; however, as we will deal with lattices with non-valuation metrics as well (particularly the supremum metric), we should better distinguish between valuation metric lattices and non-valuation metric lattices.

Proof $d_{v}(f, f)=0, d_{v}(f, g)=d_{v}(g, f)$ and property (1) are obvious. The absorption laws tell us that $f \leq f \vee g$ and $f \geq f \wedge g$ for all $f, g \in L$, hence $f \vee g \geq f \wedge g$ and isotony of $v$ yield $d_{v}(f, g) \geq 0$. Contrary to Birkhoff, we will use difference valuations to prove triangle inequality:

$$
d_{v}(f, g)=w(f, g \vee h)+w(f \wedge h, g)+w(g, f \vee h)+w(g \wedge h, f)
$$

Due to positivity of $w$ and property (2) in Proposition 51, we have

$$
\begin{aligned}
& w(f, g \vee h) \leq w(f, h) \\
& w(f \wedge h, g) \leq w(h, g) \\
& w(g, f \vee h) \leq w(g, h) \\
& w(g \wedge h, f) \leq w(h, f)
\end{aligned}
$$

And thus

$$
d_{v}(f, g) \leq w(f, h)+w(h, f)+w(h, g)+w(g, h) \leq d_{v}(f, h)+d_{v}(h, g) .
$$

We finally show that $d_{v}$ is a metric if and only if $v$ is positive. Again, we use $d_{v}(f, g)=w(f, g)+w(g, f)$ to see that $d_{v}(f, g)=0$ implies $w(f, g)=0$ and $w(g, f)=0$. Proposition [51] property (4) applies: $f \leq g$ and $g \leq f$, thus 


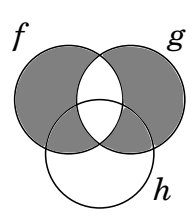

$d(f, g)$

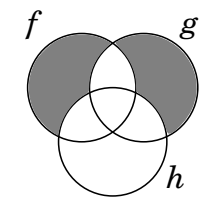

$=$

$d(f \vee h, g \vee h) \quad+$

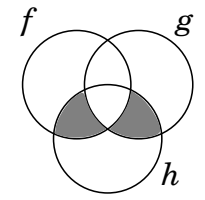

$d(f \wedge h, g \wedge h)$

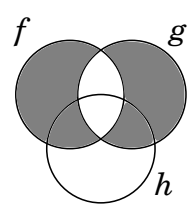

$d(f, g)$

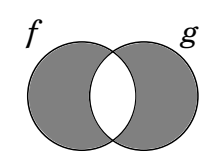

$d(f, g)$

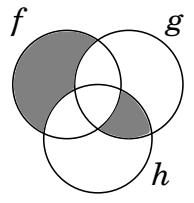

$d(f \vee(g \wedge h), f \wedge(g \vee h))$

$+d(g \vee(f \wedge h), g \wedge(f \vee h))$

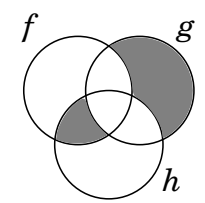

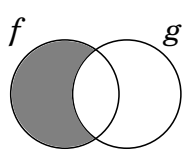

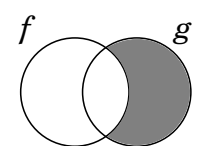
. 


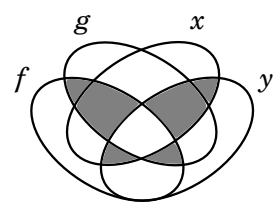

$d(f \wedge g, x \wedge y)$

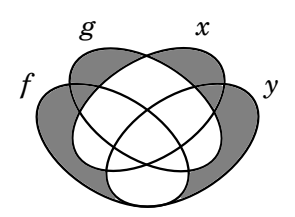

$d(f \vee g, x \vee y) \quad \leqslant$

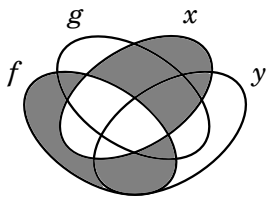

$d(f, x)$

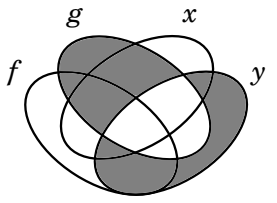

$d(g, y)$

Figure 1.4: Another property of valuation metrics, visualized with a four-set Venn diagram; a precursor to Proposition 72

Example 54

We continue with the special case $L=(\mathbb{Z}, \min , \max )$ from example 50 . Although it was not possible to create a link between the valuation $v$ and the counting measure $\mu$, the difference valuation $w$ on L provides us with a useful connection:

$$
w(n, m)=\mu(A(n) \backslash A(m))
$$

In cases of distributive lattices without least elements, it is feasible and beneficial to forget about $v$ and define $d_{v}$ directly in terms of the difference valuation. Proposition 51 shows that this is possible without loss of information.

We want to demonstrate the use of Venn diagrams to derive calculation rules in valuation metric lattices:

Proposition 55 55

Let $(L, \wedge, \vee)$ be a distributive lattice with difference valuation $w$ and corresponding valuation metric $d$. Then for all $f, g, x, y \in L$ holds:

$$
d(f \wedge g, x \wedge y)+d(f \vee g, x \vee y) \leq d(f, x)+d(f, y)
$$

Proof Figure 1.4 depicts the proposition. As the symmetry of the figure suggests, we can prove the slightly stronger statement

$$
w(f \wedge g, x \wedge y)+w(f \vee g, x \vee y) \leq w(f, x)+w(f, y)
$$

from which the thesis directly follows. Next, we decompose each term into smaller subterms with the help of the cut and absorption laws, to gain subterms of the form $w\left(a_{1} \wedge \ldots \wedge a_{n}, b_{1} \vee \ldots \vee b_{n}\right)$ in which each of $f, g, x, y$ appear at exactly one position. These are minimal terms, which each correspond to one of the fifteen minimal areas in the Venn diagram. Figure 1.4 helps us to determine along which function we have to cut. We get:

$$
\begin{aligned}
w(f \wedge g, x \wedge y)= & w(f \wedge g \wedge x, y)+w(f \wedge g \wedge y, x)+w(f \wedge g, x \vee y) \\
w(f \vee g, x \vee y)= & w(f \wedge g, x \vee y)+w(f, g \vee x \vee y)+w(g, f \vee x \vee y) \\
w(f, x)= & w(f \wedge g \wedge y, x)+w(f \wedge y, g \vee x) \\
& +w(f \wedge g, x \vee y)+w(f, g \vee x \vee y) \\
w(g, y)= & w(f \wedge g \wedge x, y)+w(g \wedge x, f \vee y) \\
& +w(f \wedge g, x \vee y)+w(g, f \vee x \vee y)
\end{aligned}
$$


It is possible to recombine the remaining subterms and express the difference of left- and right-hand side as another sum of distances - we leave this as recreation to the reader.

Example 56

If $L=\operatorname{Lip} X$, with $X$ a measure space, we may apply the Lebesgue integral to gain an isotone valuation on $L$; as $f+g=(f \wedge g)+(f \vee g)$ holds pointwise, we conclude

$$
\int f \mathrm{~d} \mu+\int g \mathrm{~d} \mu=\int(f \wedge g) \mathrm{d} \mu+\int(f \vee g) \mathrm{d} \mu .
$$

If $X$ is a Euclidean space, or a discrete space without non-trivial null sets, this valuation is positive, because any non-trivial non-negative Lipschitz function has positive Lebesgue integral. Positivity fails in cases where $X$ contains an isolated point or continuum of measure zero.

As $|f-g|=(f \vee g)-(f \wedge g)$ holds pointwise, the valuation metric $d_{v}$ equals the $L^{1}$-distance defined by

$$
d_{1}(f, g):=\int|f-g| \mathrm{d} \mu .
$$

This raises the question for which metrics $d$ on $\operatorname{Lip} X$ there is a valuation $v$, such that $d=d_{v}$. From property (2) in Lemma 53 we can easily deduce the valuation $v$. Insertion into the definition of a valuation leads to the requirement

$$
d_{v}(f, 0)+d_{v}(g, 0)=d_{v}(f \vee g, 0)+d_{v}(f \wedge g, 0) \quad \forall f, g \in \operatorname{Lip} X .
$$

In the special case of functions $f, g$ with disjoint support we have $f \vee g=f+g$, so we end up with the necessary condition

$$
f \wedge g=0 \Rightarrow d_{v}(f, 0)+d_{v}(g, 0)=d_{v}(f+g, 0) .
$$

Now it should not take us any wonder that "completely non-linear" metrics like the supremum metric or

$$
d_{p}(f, g):=\sqrt[p]{\int|f-g|^{p} \mathrm{~d} \mu}
$$

for $p>1$ are non-valuation metrics.



Yet, there are some more valuation metrics besides the $L^{1}$-metric. Let $\kappa$ : $[0, \infty) \rightarrow[0, \infty)$ be a positive valuation (i.e., strictly monotonically increasing), then

$$
v_{\mu, \kappa}(f):=\int \kappa(f(x)) \mathrm{d} \mu(x)
$$

is a positive valuation. However, the author is not aware of any valuation metric on $\operatorname{Lip} X$, which cannot be described in this way with suitable $\kappa$ and $\mu$. 


\section{$1.2 \quad$ Ultravaluation Metrics}

Lemma 58

Let $L$ be a distributive lattice, and let $w: L \times L \rightarrow[0, \infty]$ be a map which satisfies

(1) $\quad w(f, g)=0$ whenever $f \leq g$, and

(2) $\quad w(f, g)=w(f \wedge h, g) \vee w(f, g \vee h) \quad \forall f, g, h \in L$.

We call $w$ a difference ultravaluation, or just ultravaluation. Define $d_{w}(f, g):=$ $w(f, g) \vee w(g, f)$. Then $d_{w}$ is a pseudo-ultrametric $c_{+} \cdot d_{w}$ is an ultrametric $c_{+}$if and only if $w(f, g)=0 \Rightarrow f \leq g$ holds.

Proof To get from normal valuations to ultravaluations, we just replaced all occurences of "+" by " $\vee$ ". As both operations are associative and commutative, we can transfer most proofs of valuations just by replacing "+" by " $\vee$ ":

$$
\begin{aligned}
d_{w}(f, g) & =w(f, g \vee h) \vee w(f \wedge h, g) \vee w(g, f \vee h) \vee w(g \wedge h, f) \\
w(f, g \vee h) & \leq w(f, h) \text { etc. } \\
\Rightarrow \quad d_{w}(f, g) & \leq w(f, h) \vee w(h, g) \vee w(g, h) \vee w(h, f)=d_{w}(f, h) \vee d_{w}(h, g)
\end{aligned}
$$

On the other hand, contrary to the valuation case, the property $d_{v}(f, f)=0$ does not follow from property (2) - we have to conclude it from (1).

Assume $w(f, g)=0 \Rightarrow f \leq g$ holds. Let $d_{w}(f, g)=0$. This implies $w(f, g)=0$ and $w(g, f)=0$, and hence $f \leq g, g \leq f$, and $f=g$. Now assume $d_{w}$ is a metric, $f \not \leq g$, and $w(f, g)=0$. Then

$$
w(f, f \wedge g)=w(f \wedge g, f \wedge g) \vee w(f, g)=0 .
$$

Due to $f \not \leq g$, we have $f \neq f \wedge g$, hence

$$
0<d_{w}(f, f \wedge g)=w(f, f \wedge g) \vee w(f \wedge g, f)=w(f \wedge g, f) .
$$

But $f \wedge g \leq f$, contradiction.

Example 59

Let $X$ be any set, $\kappa: X \rightarrow[0, \infty]$ arbitrary and fixed, and $L$ a lattice of subsets of $X$. For $A, B \in L$ consider

$$
w(A, B):=0 \vee \sup _{x \in A \backslash B} \kappa(x) .
$$

$w$ defines an ultravaluation.

Choose $\kappa$ to be a positive constant, then the ultrametric resulting from $w$ will be the discrete metric on $X$. 


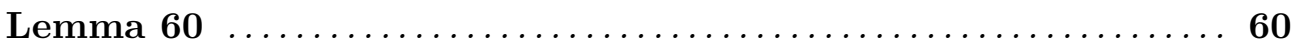

Let $X$ be finite, and let $L$ be a lattice of subsets of $X$. Then any ultravaluation on $L$ is of the form of Example 5.9.

Proof For $x \in X$ and $A, B \subseteq X$ define

$$
\begin{aligned}
\kappa(x) & :=\inf \{w(C, D): C, D \in L \text { with } x \in C, x \notin D\} \\
\text { and } \quad w^{\prime}(A, B) & :=0 \vee \sup _{y \in A \backslash B} \kappa(y) .
\end{aligned}
$$

Assume $w^{\prime}(A, B)>w(A, B)$. Then there is $y \in A \backslash B$ with $\kappa(y) \geq w(A, B)$, but this cannot happen, as one may choose $C=A$ and $D=B$. Hence, assume $w^{\prime}(A, B)<w(A, B)$. Then for all $y \in A \backslash B$ there should be $C, D \in L$ with $y \in C \backslash D$ and $w(C, D)<w(A, B)$. As

$$
w(C, D) \geq w(C \wedge A, D \vee B),
$$

we might choose without loss of generality $C \subseteq A$ and $D \supseteq B$, as choosing $C \cap A$ instead of $C$ and $D \cup B$ instead of $D$ further decreases $w(C, D)$. The cut law now yields

$$
w(A, B)=w(C \wedge D, B) \vee w(C, D) \vee w(A \vee D, B \vee C) \vee w(A, C \vee D)
$$

As $w(C, D)<w(A, B)$ by assumption, we find that at least one of $(C \cap D) \backslash B$, $(A \cup D) \backslash(B \cup C)$, and $A \backslash(C \cup D)$ must be non-empty. Choose $y^{\prime}$ out of their union and repeat the above argument for the now smaller subset. We get an infinite sequence of different elements from $X$, which is a contradiction because $X$ is finite.

\section{Example 61}

Now consider $L=\operatorname{Lip} X$, with $X$ some metric space. We represent $L$ with subsets of $X \times[0, \infty]$, as described in Example [45, to apply it to Example [59, The most canonical $\kappa$ would be $\kappa=\pi_{2}$, the projection onto $[0, \infty]$. The corresponding ultrametric on $L$ is

$$
d_{\kappa}(f, g)=0 \vee \sup \{f(x) \vee g(x) \text { with } x \in X \text { such that } f(x) \neq g(x)\} .
$$

We shall call this metric the "peak metric" on $\operatorname{Lip} X$.

Another possible choice for $\kappa$ is as follows: Choose a basepoint $x_{0} \in X$ and $\kappa(x, r):=d_{X}\left(x, x_{0}\right)$. Then $d_{\kappa}$ will describe the greatest distance from $x_{0}$ at which $f$ and $g$ still differ. Finally, $\kappa(x, r):=\exp \left(-d_{X}\left(x, x_{0}\right)\right)$ will describe the least distance from $x_{0}$ at which $f$ and $g$ differ. We will call the first case the "outer basepoint metric" and the second case the "inner basepoint metric".

An application of the lower basepoint metric is as follows: Given a free group $F$ with neutral element $x_{0}$, identify each normal subgroup $N \unlhd F$ with its characteristic function on $F$. These are 1-Lipschitz functions in the canonical word metric of $F . d_{\kappa}$ then defines a topology on $\operatorname{Lip} F$, which restricts to the Cayley topology ([dH], V.10) on the subset of normal subgroups. 


\subsection{Intervaluation Metrics}

We now integrate the supremum metric into the context of difference valuation, but not without a sincere generalization of the concept. Similar to the case of the ultravaluation, We first recognize the possibility to replace "+" in the definition of a difference valuation by any commutative and associative binary operation. But this alone will not suffice to encompass the supremum metric, we have to weaken the main property of a difference evaluation as well:

Definition 62

An intervaluation on a distributive lattice $(L, \wedge, \vee)$ is a map $w: L \rightarrow[0, \infty]$ together with a commutative and associative binary operation $\circ_{w}:[0, \infty] \times$ $[0, \infty] \rightarrow[0, \infty]$, such that the following properties hold:

1. $r \circ_{w} 0=0 \circ_{w} r=r$

2. $r \circ_{w} t \leq(r+s) \circ_{w}(t+u) \leq\left(r \circ_{w} t\right)+\left(s \circ_{w} u\right)$

3. $r \vee s \leq r \circ_{w} s \quad$ (follows from (1) and (2))

4. $f \leq g \Rightarrow w(f, g)=0$

5. $w(f, g \vee h) \circ_{w} w(f \wedge h, g) \leq w(f, g) \leq w(f, g \vee h)+w(f \wedge h, g)$ (left and right modular inequality, or cut law)

for all $f, g, h \in L$ and $r, s, t, u \in[0, \infty]$. The corresponding intervaluation metric then is defined to be

$$
d_{w}(f, g):=w(f, g) \circ_{w} w(g, f) .
$$

The intervaluation is positive if

$$
w(f, g)=0 \Rightarrow f \leq g .
$$

Proposition 63

An intervaluation $w$ on $L$ and its metric $d_{w}$ always fulfill:

1. $w(f, g)=w(f \vee g, g)=w(f, f \wedge g)=d_{w}(f \vee g, g) \quad \forall f, g \in L$.

2. $d_{w}$ is a pseudo-metric + .

3. $d_{w}$ is a metric $c_{+}$if and only if $w$ is positive.

Proof (1) We choose $h=f$ or $h=g$ in both modular inequalities:

$$
\begin{aligned}
& 0 \circ_{w} w(f, g) \leq w(f \vee g, g) \leq 0+w(f, g) \\
& w(f, g) \circ_{w} 0 \leq w(f, g \wedge f) \leq w(f, g)+0 \\
& \text { and } \quad d_{w}(f \vee g, g)=w(f \vee g, g) \circ_{w} 0=w(f, g) .
\end{aligned}
$$


(2) From the definition we see $d_{w}(f, g) \geq 0$ and $d_{w}(f, f)=0$ for all $f, g \in L$. As $\circ_{w}$ is commutative, $d_{w}$ is symmetric.

$$
\begin{aligned}
d_{w}(f, g) & =w(f, g) \circ_{w} w(g, f) \\
& \leq(w(f \wedge h, g)+w(f, g \vee h)) \circ_{w}(w(g \wedge h, f)+w(g, f \vee h)) \\
& \left.\leq(w(h, g)+w(f, h)) \circ_{w}(w(h, f)+w(g, h))\right) \\
& \left.=(w(f, h)+w(h, g)) \circ_{w}(w(h, f)+w(g, h))\right) \\
& \left.\leq\left(w(f, h) \circ_{w} w(h, f)\right)+\left(w(h, g) \circ_{w} w(g, h)\right)\right) \\
& =d_{w}(f, h)+d_{w}(h, g)
\end{aligned}
$$

$(\mathbf{3}$, " $\Rightarrow ")$ Assume $0=w(f, g)=w(f, f \wedge g)$. Then $d_{w}(f, f \wedge g)=0+0=0$. As $d_{w}$ is a metric, we have $f=f \wedge g$, so $f \leq g$.

$(\mathbf{3}$, " $\Leftarrow ") ~ d_{w}(f, g)=0$ implies $w(f, g)=0$ and $w(g, f)=0$, hence $f \leq g \leq f$, and $f=g$.

The definition of intervaluations is chosen to generalize difference valuations and ultravaluations, while maintaining as many inequalities as possible. As those calculation laws derived from Venn-diagrams hold for difference valuations and ultravaluations likewise, which both work as the extremal cases of intervaluations, it is a worthwhile endeavour to figure out those laws that still hold for intervaluation metrics, which contain lots of interesting metrics for Lipschitz function spaces.

Lemma 64 64

Let $L$ be a distributive lattice with intervaluation metric $+d$. Define a polynomial in $L$ as an expression of finitely many variables in $L$, linked by $\vee$ and $\wedge$. Define $a$ difference term in $L$ to be a term of the form $w(x, y)$, where $x$ and $y$ are polynomials in $L$. Define a difference polynomial in $L$ as a term built from finitely many difference terms, linked by addition. Let $\mathcal{T}_{1}$ and $\mathcal{T}_{2}$ be difference terms. Let $f_{1}$ to $f_{N}$ be the set of all variables occuring in $\mathcal{T}_{1}$ and $\mathcal{T}_{2}$. Draw a Venn diagram with $f_{1}$ to $f_{N}$ as basis, and let $\pi$ be the correspondence between the subsets of the Venn diagram and polynomials of $f_{1}$ to $f_{N}$ in L. Define $\rho(x, y):=\pi(x) \backslash \pi(y)$. Define $\pi\left(\mathcal{T}_{1}\right)$ to be the union of all $\rho(x, y)$ for which $w(x, y)$ appears in $\mathcal{T}_{1}$. If $\pi\left(\mathcal{T}_{1}\right) \subseteq \pi\left(T_{2}\right)$, then $\mathcal{T}_{1} \leq\left(2^{N}-1\right) \cdot \mathcal{T}_{2}$.

Proof Call the $2^{N}-1$ minimal subsets in the Venn diagram atoms. Each atom $A$ is uniquely described by a non-empty subset $S$ of $\left\{f_{1}, \ldots, f_{N}\right\}$, such that

$$
A=\left(\bigcap_{f_{j} \in S} \pi\left(f_{j}\right)\right) \backslash\left(\bigcup_{f_{j} \notin S} \pi\left(f_{j}\right)\right),
$$

and hence $A=\rho\left(\bigwedge_{S} f_{j}, \bigvee_{S^{c}} f_{j}\right)$. On the other hand, each difference term $w(x, y)$ can be uniquely chopped down into corresponding atoms of the form $w\left(\bigwedge_{S} f_{j}, \bigvee_{S^{c}} f_{j}\right)$ as well, by repeatedly using the cut law, up to $N$ times (for 
each variable once; as $\wedge$ and $\vee$ distribute over each other as well as over themselves, the absorption law reduces each polynomial to a polynomial of $\wedge$ or $\vee$ only); uniqueness follows from commutativity. By Definition 62 we conclude:

maximum of up to $\left(2^{N}-1\right)$ atoms $\leq w(x, y) \leq \operatorname{sum}$ of up to $\left(2^{N}-1\right)$ atoms

As $\sum_{j \in J} a_{j} \leq \# J \cdot \max _{j \in J} a_{j}$ for any finite index set $J$ and real numbers $a_{j}$, we have

sum of atoms $\leq\left(2^{N}-1\right) \cdot w(x, y) \leq\left(2^{N}-1\right) \cdot($ sum of atoms $)$

Correspondingly, each difference term $\mathcal{T}$ can be bounded from above and below by multiples of the atoms of its constituents. If $\pi\left(\mathcal{T}_{1}\right) \subseteq \pi\left(\mathcal{T}_{2}\right)$, then each atom of the left-hand Venn diagram occurs in the right-hand Venn diagram as well, hence the sum of atoms in $\mathcal{T}_{1}$ is a subsum of the sum of atoms in $\mathcal{T}_{2}$. We then follow

$$
\mathcal{T}_{1} \leq \text { sum of } \mathcal{T}_{1} \text {-atoms } \leq \text { sum of } \mathcal{T}_{2} \text {-atoms } \leq\left(2^{N}-1\right) \cdot \mathcal{T}_{2} .
$$

\section{Example 65}

There are several possible choices for the commutative and associative binary operation $\circ_{w}$ in Definition 62. Choosing addition leads directly to the definition of valuations. The next important choice is the maximum operation: Properties (1) and (3) are obviously fulfilled, the left side of (2) as well. (2.right) needs some short consideration: As + distributes over $\vee$, the right-hand side equals

$$
(r \vee t)+(s \vee u)=(r+s) \vee(r+u) \vee(t+s) \vee(t+u)
$$

which is greater or equal $(r+s) \vee(t+u)$ for all $r, s, t, u \in[0, \infty]$.

Each norm $\|\cdot\|$ on $\mathbb{R}^{2}$ with certain normalization properties qualifies as an operation $\circ_{w}$ via $r \circ_{w} s:=\|(r, s)\|$. This accounts for the $p$-norms:

$$
r \circ_{p} s:=\|(r, s)\|_{p}:=\sqrt[p]{r^{p}+s^{p}}
$$

for $p \in[1, \infty)$. Again, properties (1), (2.left) and (3) of Definition 62 are trivial. Property (2.right) is the triangle inequality of the p-norms (i.e. a special case of the Minkowski inequality [Wr]).

Given any metric $d$ on $L$ we may define $w_{d}(f, g):=d(f \vee g, g)$ and deduce $\circ_{w}$ from $d(f, g)=w_{d}(f, g) \circ_{w} w_{d}(g, f)$. The operation $\circ_{w}$ must be commutative due to the symmetry of $d_{w}$. From the remaining properties of Definition 62, property (4) follows directly from $d(g, g)=0$, while the rest is less obvious.

Example 66

The standard metric $c_{+}$on $[0, \infty]$ is an intervaluation metric + with

$$
w(r, s):=\left\{\begin{array}{ll}
0 \vee(r-s) & : s<\infty \\
0 & : s=\infty
\end{array} \quad \forall r, s \in[0, \infty] .\right.
$$


However, one may freely choose $o_{w}$ to be addition or maximum. To prove the cut law for both choices, it suffices to show

$$
0 \vee(r-s)=(0 \vee(r-(s \vee t)))+(0 \vee((r \wedge t)-s))) .
$$

For this, we make use of $a+b=(a \wedge b)+(a \vee b)$ with $a=r \wedge s$ and $b=r \wedge t$, then add $r$ to both sides, rearrange and apply $x-(x \wedge y)=0 \vee(x-y)$.

Example 67

Let $(X, \mu)$ be a measure space, $L=\operatorname{Lip} X$, and $p \in(1, \infty)$ arbitrary. Define $r \circ_{w} s=\left(r^{p}+s^{p}\right)^{1 / p}$, and

$$
w(f, g):=\sqrt[p]{\int|f-(f \wedge g)|^{p} \mathrm{~d} \mu} .
$$

As $|r-(r \wedge s)|^{p}+|s-(r \wedge s)|^{p}=|r-s|^{p}$ for all $r, s \in[0, \infty]\left(\right.$ with $\left.\infty^{p}:=\infty\right)$, the corresponding (pseudo-)metric $c_{+}$is just the $L^{p}$-metric $c_{+}$

$$
d_{p}(f, g)=\sqrt[p]{\int|f-g|^{p} \mathrm{~d} \mu} .
$$

Properties (1)-(3) of Definition [62 follow from Example [65, (4) is trivial. The left cut law can be shown by pointwise analysis and case distinction ( $h \leq g$ vs. $h>g$ ), the right cut law follows from Example [66 and the Minkowski inequality. $d_{p}$ might be a pseudo-metric $c_{+}$, depending on $\mu$.

Example 68

Here is a minimal example for a non-intervaluation metric: Take $L=\{a, b, c\}$ with $a<b<c$, and $d(a, c)=1, d(a, b)=2, d(b, c)=3$. Then $w(c, a)=1$, although $w(c \wedge b, a)=2$ and $w(c, a \vee b)=3$, which both contradict the cut law and Proposition [63. 1, no matter what $\circ_{w}$ is.

Example 69

The Lipschitz constant provides a much more interesting example for a nonintervaluation metric. Let $X$ be an arbitrary true metric space, and $L$ a complete lattice of functions $f: X \rightarrow \mathbb{R}$. The Lipschitz constant of a function $f \in L$ and the corresponding pseudo-metric $c_{+}$are given by

$$
\begin{aligned}
\operatorname{LC}(f) & :=\sup _{x, y \in X} \frac{|f(x)-f(y)|}{d(x, y)} \\
d_{\mathrm{LC}}(f, g) & :=\operatorname{LC}(f-g) .
\end{aligned}
$$

They are used by [Wv] as ingredient to the utilized norm, called Lipschitz norm, which is defined as $\|f\|_{L}:=\|f\|_{\infty} \vee \mathrm{LC}(f)$. However, neither defines an intervaluation: Although Weaver shows in his Proposition 1.5.5 that LC fulfills a modular inequality for ultravaluations

$$
\mathrm{LC}(f \vee g) \vee \mathrm{LC}(f \wedge g) \leq \mathrm{LC}(f) \vee \mathrm{LC}(g)
$$


the inverse inequality is wrong, as there is no bound to $\mathrm{LC}(f)$ by any combination of $\mathrm{LC}(f \wedge g)$ and $\mathrm{LC}(f \vee g)$. To see this, consider the two-point-space $X=\{a, b\}$ of diameter $l<1$, and the Lipschitz-functions $f=(0, l)$ and $g=(l, 0)$. Then $\operatorname{LC}(f)=\|f\|_{L}=1$, but $\operatorname{LC}(f \wedge g)=\operatorname{LC}(f \vee g)=0$ and $\|\cdot\|_{L}=l$ in both cases.

Correspondingly, the cut law is explicitly violated by $d_{\mathrm{LC}}$, as one can see when $f$ and $g$ are two different constant functions, and $h$ crosses them both.

We now concentrate on the special case of the supremum metric.

Proposition 70

Let $Z$ be a distributive lattice with intervaluation metric $c_{+} d$ (with corresponding $w_{d}$ and $\left.\circ_{d}\right)$, with $r \circ_{d} s=r \vee s$ for all $r, s \in[0, \infty]$. Let $X$ be an arbitrary space, and $L$ a complete lattice of functions $f: X \rightarrow Z$ with pointwise infima and suprema. Then

$$
w_{\infty}(f, g):=\bigvee_{x \in X} w_{d}(f(x), g(x))
$$

defines an intervaluation metric on $L$ with $r \circ_{\infty} s=r \vee s$ for all $r, s \in[0, \infty]$, which equals the supremum metric $d_{+}$.

Proof The left inequality of the cut law is trivial. For the right side we have to use that a supremum of sums is less than or equal to a sum of suprema, which in turn follows from complete distributivity:

$$
\begin{aligned}
\bigvee_{x \in X} w_{d}(f x, g x) & \leq \bigvee_{x \in X}\left(w_{d}(f x,(g \vee h)(x))+w_{d}((f \wedge h)(x), g x)\right) \\
& \leq \bigvee_{x \in X} w_{d}(f x,(g \vee h)(x))+\bigvee_{x \in X} w_{d}((f \wedge h)(x), g x)
\end{aligned}
$$

Corollary 71

Let $X$ be any metric $c_{+}$space. The supremum metric $d_{+}$is an intervaluation

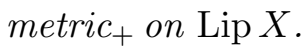

Proof We have seen in Proposition 43 that $\operatorname{Lip} X$ is a complete lattice. We easily find $r \circ_{d_{\infty}} s=r \vee s$ and

$w_{d_{\infty}}(f, g)=\bigvee_{x \in X}|f(x)-(f \wedge g)(x)|=0 \vee \bigvee_{x \in X}(f(x)-g(x))$,

which is the intervaluation metric + of Proposition [70 applied to Example 66.

Apart from all laws which we may derive from Venn diagrams, the supremum metric on $\operatorname{Lip} X$ provides yet another interesting law, which is an adaptation and generalization of Proposition 55. 
Proposition 72 72

For $f_{j}, g_{j}$ arbitrary set theoretic functions $X \rightarrow[0, \infty], j \in J, J$ some arbitrary index set, holds:

$$
\begin{aligned}
& d_{\infty}\left(\bigwedge_{j \in J} f_{j}, \bigwedge_{j \in J} g_{j}\right) \leq \bigvee_{j \in J} d_{\infty}\left(f_{j}, g_{j}\right) \\
& d_{\infty}\left(\bigvee_{j \in J} f_{j}, \bigvee_{j \in J} g_{j}\right) \leq \bigvee_{j \in J} d_{\infty}\left(f_{j}, g_{j}\right)
\end{aligned}
$$

Proof For $J=\emptyset$, both inequalities are trivial. Assume $J \neq \emptyset$. As $\bigvee_{j \in J}$ and $\bigvee_{x \in X}$ commute, it suffices to show

$$
\begin{aligned}
& d_{\infty}\left(\bigwedge x_{j}, \bigwedge y_{j}\right) \leq \bigvee d_{\infty}\left(x_{j}, y_{j}\right) \\
& d_{\infty}\left(\bigvee x_{j}, \bigvee y_{j}\right) \leq \bigvee d_{\infty}\left(x_{j}, y_{j}\right)
\end{aligned}
$$

for any $x_{j}, y_{j} \in[0, \infty]$.

First we handle infinities. First inequality: Assume there is $j$ with $x_{j}=y_{j}=\infty$. We can ignore all such $j$ 's from $J$, unless all $x_{j}$ and $y_{j}$ are $\infty$. In this case on both sides are zeros. Now assume $x_{j}=\infty \neq y_{j}$. Then $\infty$ appears on the right side and trivializes the inequality. So we can restrict to finite $x_{j}$ and $y_{j}$. Note that $\bigwedge_{j} x_{j}=\infty$ can only happen when all $x_{j}=\infty$.

Second inequality: Assume $\bigvee_{j} x_{j}=\infty$, but $\bigvee_{j} y_{j}$ is finite. Then there is an upper bound for $y_{j}$ but not for $x_{j}$. Hence the right side becomes infinite, too. Note that infinite $x_{j}$ or $y_{j}$ automatically lead to infinite $\bigvee_{j} x_{j}$ or $\bigvee_{j} y_{j}$, respectively.

Without restriction let $\bigwedge_{j} x_{j} \geq \bigwedge_{j} y_{j}$, and let $M:=\bigvee_{j} d\left(x_{j}, y_{j}\right)$. Let $\delta>0$ be arbitrary. Then there is an $m \in J$ with $y_{m} \leq \bigwedge_{j} y_{j}+\delta$. Furthermore we have $d\left(x_{m}, y_{m}\right) \leq M$, hence $y_{m} \geq x_{m}-M$. Altogether:

$$
\bigwedge x_{j} \leq x_{m} \leq \bigwedge y_{j}+M+\delta
$$

Now let $\delta \rightarrow 0$. The other inequality works the same way.

\subsection{Complete Metric/Lattice-Irreducibility}

Recall Definition 36] of a join-irreducible element $p$ in a lattice $L$ :

$$
p=f \vee g \Rightarrow p=f \quad \text { or } \quad p=g \quad \forall f, g \in L
$$

Let $L$ be equipped with the discrete metric $d_{\text {dis }}$. Then the above property is equivalent to the following:

$$
d_{\text {dis }}(p, f) \wedge \quad d_{\text {dis }}(p, g) \leq d_{\text {dis }}(p, f \vee g) \quad \forall f, g \in L
$$


In the same sense, $p$ is completely irreducible if and only if

$$
\bigwedge_{j \in J} d_{\text {dis }}\left(p, f_{j}\right) \leq d_{\text {dis }}\left(p, \bigvee_{j \in J} f_{j}\right) \quad \forall\left(f_{j}\right)_{j \in J} \subseteq L, J \neq \emptyset
$$

Definition $\mathbf{7 3}$

Let $L$ be a complete lattice with intervaluation metric $+d$. We call $p \in L$ completely ml-irreducible if the following equivalent conditions hold:

1. $\forall\left(f_{j}\right)_{j \in J} \subseteq L$, with $J$ an arbitrary non-empty index set:

$$
\bigwedge_{j \in J} d\left(p, f_{j}\right) \leq d\left(p, \bigvee_{j \in J} f_{j}\right)
$$

2. $\forall\left(f_{j}\right)_{j \in J} \subseteq L, J$ an arbitrary non-empty index set, and $\forall R \in[0, \infty]$ :

$$
d\left(p, \bigvee_{j \in J} f_{j}\right) \leq R \Rightarrow \forall \delta>0 \exists j \in J: d\left(p, f_{j}\right) \leq R+\delta
$$

(This results from expanding the infimum in (1).)

Let $0 \in L$ be the least element in $L$, then a bounded completely ml-irreducible element $p \in L$ is a completely ml-irreducible element with finite distance to 0. Denote the subset of $L$ of all [bounded] completely ml-irreducible elements with $\operatorname{cmli}(L)[\mathrm{bcmli}(L)]$.

Proposition $\mathbf{7 4}$

Let $L$ be metrically closed, then each completely ml-irreducible element is joinirreducible. It is not necessarily completely join-irreducible (see Definition [36).

Proof Use $R=0$ and that a set of two elements is compact. For a counterexample to complete join-irreducibility, let $L=[0,1]$ with standard metric, supremum and infimum. Take $f_{n}=1-1 / n, n \in \mathbb{N}^{*}$, then $p=1=\bigvee f_{n}$, hence $p$ is not completely join-irreducible. Still, it is completely ml-irreducible: Any sequence of real numbers $f_{n}$ with $p=\bigvee f_{n}$ must converge to $p$ from below, hence $\bigwedge d\left(p, f_{n}\right)=0$.

Let $L$ be a complete lattice with intervaluation metric $d$, and let $L$ be metrically complete. Then $\operatorname{cmli}(L)$ is topologically closed. If 0 is the least element of $L$, then $\operatorname{bcmli}(L)$ is topologically closed, and $0 \in \operatorname{bcmli}(L)$. 
Proof Let $\left(p_{n}\right) \subseteq \operatorname{cmli}(L), n \in \mathbb{N}^{*}$ be some sequence of completely ml-irreducible elements converging to $p \in L$, and $\left(f_{j}\right)_{j \in J}$ any non-empty family in $L$. Then for any $n \in \mathbb{N}^{*}$ holds

$$
\begin{aligned}
d\left(p, \bigvee f_{j}\right) & \geq d\left(p_{n}, \bigvee f_{j}\right)-d\left(p, p_{n}\right) \\
& \geq \bigwedge d\left(p_{n}, f_{j}\right)-d\left(p, p_{n}\right) \\
& \geq \bigwedge\left(d\left(p, f_{j}\right)-d\left(p, p_{n}\right)\right)-d\left(p, p_{n}\right) \\
& \geq \bigwedge d\left(p, f_{j}\right)-\underbrace{2 d\left(p, p_{n}\right)}_{\rightarrow 0},
\end{aligned}
$$

i.e. the element $p$ is completely ml-irreducible.

Any component of $L$ is topologically closed, in particular the component of 0 , hence the intersection with $\operatorname{cmli}(L)$ is closed as well. Furthermore, we have for any $k \in J \neq \emptyset$ :

$$
\begin{aligned}
d\left(0, \bigvee f_{j}\right) & =w_{d}\left(\bigvee f_{j}, 0\right) \quad \mid \text { idempotency } \\
& =w_{d}\left(f_{k} \vee \bigvee f_{j}, 0\right) \quad \mid \text { cut along } f_{k} \\
& \geq w_{d}\left(f_{k}, 0\right) o_{w} w_{d}\left(\bigvee f_{j}, f_{k}\right) \\
& \geq w_{d}\left(f_{k}, 0\right)=d\left(0, f_{k}\right)
\end{aligned}
$$

and thus $\bigwedge d\left(0, f_{j}\right) \leq d\left(0, f_{k}\right) \leq d\left(0, \bigvee f_{j}\right)$

Definition 76

Let $L$ be a lattice with metric $d, R \geq 0$ arbitrary. We define an $R$-base of $L$ to be a subset $B \subseteq L$ such that for any $f \in L$ there is $\left(b_{j}\right)_{j \in J} \subseteq B$, J an arbitrary non-empty index set, such that $d\left(f, \bigvee_{j \in J} b_{j}\right) \leq R$. A base simply is a 0 -base. Given a least element $0 \in L, a$ bounded $R$-base is an $R$-base of $L$ which is a subset of the component of 0 .

Example 77

Let $L=[0, \infty]$ with standard metric $c_{+} d$ (modulus of the difference). Then one easily calculates $\operatorname{cmli}(L)=\operatorname{bcmli}(L)=[0, \infty)$. Furthermore, any metrically dense subset $B$ of $[0, \infty)$ is a bounded base, in particular, we have sequences $\left(b_{j}\right) \subseteq B$ with $\bigvee b_{j}=\infty$, although $d\left(b_{j}, \infty\right)=\infty$, and thus $b_{j} \nrightarrow \infty$ ind.

Proposition 78

Consider an $R$-base $B$ of a complete lattice $L$ with intervaluation metric $+d$, $R \geq 0$. Then for each $\delta>0, \operatorname{cmli}(L)$ is in the $(R+\delta)$-ball around $B$. In particular, if $R=0, \operatorname{cmli}(L)$ lies in the metrical closure of $B$. 
Proof Let $p \in \operatorname{cmli}(L)$ be arbitrary. As $B$ is an $R$-base, there are $b_{j} \in B$, $j \in J \neq \emptyset$, such that

$$
d\left(p, \bigvee_{j \in J} b_{j}\right) \leq R
$$

From Definition $\left[73\right.$ we infer that there is a sequence $\left(c_{k}\right) \subseteq B, k \in K \subseteq J$ whose distances to $p$ converge to $R$. If $R=0$, the sequence $\left(c_{j}\right)$ metrically converges to $p$.

\section{Example 79}

It is easy to see that, if $B$ is a base, and $b \in B$ not a join-irreducible element, then $B \backslash\{b\}$ is a base as well (if $b=f \vee g, f$ and $g$ are joins of elements of $B$, and as $f, g<b, b$ is not part of these joins). Using the Lemma of Zorn, it is possible to deduce that the subset of all join-irreducible elements constitutes a base for any sufficiently nice lattice.

Unfortunately, this is not the case with ml-irreducible elements: Let $L^{\prime}$ be the completely distributive complete lattice $[0,3] \times[0,2]$ with componentwise supremum and infimum, and with supremum metric. Then consider the sublattice $L \subseteq L^{\prime}$ formed by the five elements

$$
L:=\{(0,0),(1,0),(0,1),(1,1),(2,2)\} .
$$

We find $\operatorname{cmli}(L)=\{(0,0),(1,0),(0,1)\}$, as $(1,1)=(1,0) \vee(0,1) . p=$ $(2,2)$ is join-irreducible in this lattice, but not ml-irreducible: Take $f_{1}=(1,0)$, $f_{2}=(0,1)$, then $\bigwedge d\left(p, f_{j}\right)=2$, but $d\left(p, \bigvee f_{j}\right)=1$. Nevertheless, $(2,2)$ must be part of any 0 -base of $L$. 


\section{Chapter 2}

\section{Rough Isometries of Lipschitz Function Spaces}

\subsection{Smoothening of Lipschitz Functions}

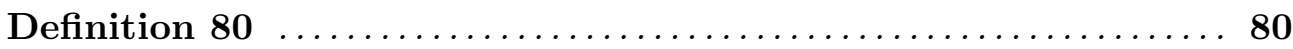

Let $X$ and $Z$ be metric $c_{+}$spaces, and $\epsilon, K \geq 0$ fixed. We define the $Z$-valued $K$-Lipschitz and $(K, \epsilon)$-Lipschitz functions on $X$ by:

$$
\begin{aligned}
\operatorname{Lip}_{K}(X, Z) & :=\{f: X \rightarrow Z: \forall x, y \in X: d(f x, f y) \leq K d(x, y)\} \\
\operatorname{Lip}_{K, \epsilon}(X, Z) & :=\{f: X \rightarrow Z: \forall x, y \in X: d(f x, f y) \leq K d(x, y)+\epsilon\}
\end{aligned}
$$

Later on we will restrict to $Z=\mathbb{R}$ and $Z=[0, \infty]$.

Given two metric + spaces $X, Y$ and a $(\lambda, \epsilon)$-quasi-isometry $\eta: X \rightarrow Y$, we can push $\eta$ to a map between the Lipschitz function spaces

$$
\begin{aligned}
\eta^{*}: \operatorname{Lip}_{K, \delta}(Y, Z) & \rightarrow \operatorname{Lip}_{K \cdot \lambda, K \cdot \epsilon+\delta}(X, Z) \\
f & \mapsto f \circ \eta .
\end{aligned}
$$

Using the supremum metric (aka. $L^{\infty}$-metric) $d_{\infty}$ on Lipschitz functions, we easily see that $\eta^{*}$ is a $(2 K \epsilon+2 \delta)$-isometric embedding of $\operatorname{Lip}_{K, \delta}(Y, Z)$ into $\operatorname{Lip}_{K \cdot \lambda, K \cdot \epsilon+\delta}(X, Z)$ : Obviously, we have

$$
d(f \eta x, g \eta x) \leq d_{\infty}(f, g) \quad \forall x \in X .
$$

On the other hand, for each $0 \leq D<d_{\infty}(f, g)$ we may find $y \in Y$ with $d(f y, g y) \geq D$, and $x \in X$ with $d(\eta x, y) \leq \epsilon$. Hence,

$$
\begin{aligned}
d(f \eta x, g \eta x) & \geq d(f y, g y)-d(f \eta x, f y)-d(g \eta x, g y) \\
& \geq D-2 K \epsilon-2 \delta .
\end{aligned}
$$

Taking the supremum over $x$, we conclude $d_{\infty}\left(\eta^{*} f, \eta^{*} g\right) \geq d_{\infty}(f, g)-2 K \epsilon-2 \delta$.

Question Is $\eta^{*}$ even a quasi-isometry? 
We may split this problem into two special cases: The rough case with $\lambda=1$. And the bilipschitz case $\epsilon=0$, for which we can give an easy counterexample: Let $X$ be $\mathbb{R}^{2}$ with Euclidean metric, and let $Y$ be $\mathbb{R}^{2}$ with supremum metric. The identity is a 2-bilipschitz bijection. Let $Z=\mathbb{R}$ and $f: X \rightarrow Z,\left(x_{1}, x_{2}\right) \mapsto$ $\sqrt{x_{1}^{2}+x_{2}^{2}}$, i.e. $f(x)=|x|_{X}$, and $f \in \operatorname{Lip}_{1}(X, Z)$. In particular, for points $x$ with $x_{1}=x_{2}$ we find $f(x)=\sqrt{2} \cdot x_{1}$. But for $g \in \operatorname{Lip}_{1}(Y, Z)$ must hold

$$
|g(x)-g(0)| \leq \sup \left\{x_{1}, x_{2}\right\}
$$

such that with $x_{1} \rightarrow \infty, f$ and $g$ have infinite distance from each other. One would argue that in this case, we have to choose $g \in \operatorname{Lip}_{\sqrt{2}}(Y, Z)$. But then, we may choose points $x=\left(x_{1}, 0\right)$ to see that the function $\left(x_{1}, x_{2}\right) \mapsto \sqrt{2} \cdot|x|_{Y}$ has infinite distance to any $f \in \operatorname{Lip}_{1}(X, Z)$. To solve this problem, one has to generalize Lipschitz functions to allow for varying Lipschitz constants - however, our main focus lies on the rough isometry case $\lambda=1$. As

$$
\operatorname{Lip}_{K}(X, Z) \subseteq \operatorname{Lip}_{K, \epsilon}(X, Z)
$$

for any $\epsilon \geq 0$, we may reformulate our question in the following way:

\section{Question}

How $L^{\infty}$-dense is the subset of all K-Lipschitz functions in the metric $c_{+}$space of $(K, \epsilon)$-Lipschitz functions?

We will next give an answer to this problem in the special case $Z=\mathbb{R}$. A similar result for continuous functions is given by Petersen in $[\mathrm{P}$, section 4 .

Lemma 81

Let $X$ be a separable metric space $K, \epsilon>0$ and let $f: X \rightarrow \mathbb{R}$ be a function with

$$
|f x-f y| \leq K \cdot d(x, y)+\epsilon .
$$

Then there is a K-Lipschitz function $g: X \rightarrow \mathbb{R}$ with

$$
d_{\infty}(f, g) \leq 2 \epsilon .
$$

Proof Let $f: X \rightarrow \mathbb{R}$ be a $(K, \epsilon)$-Lipschitz function, $K, \epsilon \geq 0$. Let $\left\{a_{j}\right\}_{j \in \mathbb{N}^{*}} \subseteq$ $X$ be a countable dense subset. Define $g\left(a_{1}\right):=f\left(a_{1}\right)$. Now define inductively and prove by induction the following:

$$
I_{j}:=\bigcap_{k=1}^{j-1}\left[g\left(a_{k}\right)-K \cdot d\left(a_{j}, a_{k}\right), g\left(a_{k}\right)+K \cdot d\left(a_{j}, a_{k}\right)\right]
$$




$$
g\left(a_{j}\right):=\left\{\begin{array}{cl}
f\left(a_{j}\right) & : f\left(a_{j}\right) \in I_{j} \\
\min I_{j} & : f\left(a_{j}\right) \leq \min I_{j} \\
\max I_{j} & : f\left(a_{j}\right) \geq \max I_{j}
\end{array}\right.
$$

- (a): $\left.g\right|_{\left\{a_{1}, \ldots, a_{j}\right\}}$ is $K$-Lipschitz.

- (b): $\left|f\left(a_{j}\right)-g\left(a_{j}\right)\right| \leq \epsilon$.

We see that (a) and (b) are trivially fulfilled for $j=1$. At first we have to show that $I_{j} \neq \emptyset$ : As $\left.g\right|_{\left\{a_{1}, \ldots, a_{j-1}\right\}}$ is $K$-Lipschitz, we know for all $m, n<j$ :

$$
\begin{aligned}
& g\left(a_{m}\right)-g\left(a_{n}\right) \leq\left|g\left(a_{m}\right)-g\left(a_{n}\right)\right| \leq K \cdot d\left(a_{m}, a_{n}\right) \\
\Rightarrow \quad & g\left(a_{m}\right)-g\left(a_{n}\right) \leq K \cdot d\left(a_{m}, a_{j}\right)+K \cdot d\left(a_{n}, a_{j}\right) \\
\Rightarrow \quad & g\left(a_{m}\right)-K \cdot d\left(a_{m}, a_{j}\right) \leq g\left(a_{n}\right)+K \cdot d\left(a_{n}, a_{j}\right) \quad \forall m, n<j
\end{aligned}
$$

Thus $I_{j}$ is not empty and we can define $g\left(a_{j}\right)$ as above. Because of $g\left(a_{j}\right) \in I_{j}$, we have

$$
\left|g\left(a_{j}\right)-g\left(a_{k}\right)\right| \leq K \cdot d\left(a_{j}, a_{k}\right) \quad \forall k<j
$$

and together with the Lipschitz property of $g$ on $\left\{a_{1}, \ldots, a_{j-1}\right\}$ we see that $g$ is also $K$-Lipschitz on $\left\{a_{1}, \ldots, a_{j}\right\}$.

Finally, we show $\left|f\left(a_{j}\right)-g\left(a_{j}\right)\right| \leq \epsilon$. If $f\left(a_{j}\right) \in I_{j}$, we have nothing to show. Assume the second case: $f\left(a_{j}\right) \leq \min I_{j}$ and $g\left(a_{j}\right)=\min I_{j}$. Let $n$ be such that

$$
f\left(a_{j}\right) \leq g\left(a_{j}\right)=\min I_{j}=g\left(a_{n}\right)-K \cdot d\left(a_{n}, a_{j}\right)
$$

By definition of $I_{j}$ we have

$$
g\left(a_{n}\right)-K \cdot d\left(a_{n}, a_{j}\right) \quad \geq g\left(a_{m}\right)-K \cdot d\left(a_{m}, a_{j}\right) \quad \forall m<j
$$

for some $n$, and we choose $n$ to be the least possible index with this property. Furthermore, we have

$$
\begin{aligned}
\left|f\left(a_{j}\right)-f\left(a_{n}\right)\right| & \leq K \cdot d\left(a_{j}, a_{n}\right)+\epsilon \\
\Rightarrow \quad f\left(a_{j}\right) & \geq f\left(a_{n}\right)-K \cdot d\left(a_{j}, a_{n}\right)-\epsilon .
\end{aligned}
$$

We use this to calculate

$$
\begin{aligned}
0 \leq g\left(a_{j}\right)-f\left(a_{j}\right) & =g\left(a_{n}\right)-K \cdot d\left(a_{n}, a_{j}\right)-f\left(a_{j}\right) \\
& \leq g\left(a_{n}\right)-K \cdot d\left(a_{n}, a_{j}\right)-f\left(a_{n}\right)+K \cdot d\left(a_{n}, a_{j}\right)+\epsilon \\
& \leq g\left(a_{n}\right)-f\left(a_{n}\right)+\epsilon .
\end{aligned}
$$

Now assume $g\left(a_{n}\right)>f\left(a_{n}\right)$. Then there is some $m<n<j$ with

$$
\begin{aligned}
g\left(a_{n}\right) & =g\left(a_{m}\right)-K \cdot d\left(a_{n}, a_{m}\right) \\
\Rightarrow \quad g\left(a_{n}\right)-K \cdot d\left(a_{n}, a_{j}\right) & =g\left(a_{m}\right)-K \cdot d\left(a_{n}, a_{m}\right)-K \cdot d\left(a_{n}, a_{j}\right) \\
& \leq g\left(a_{m}\right)-K \cdot d\left(a_{m}, a_{j}\right)
\end{aligned}
$$


which contradicts the minimality of $n$. Hence we have $g\left(a_{n}\right) \leq f\left(a_{n}\right)$ and $\left|g\left(a_{j}\right)-f\left(a_{j}\right)\right| \leq \epsilon$.

The third case $\left(f\left(a_{j}\right)>g\left(a_{j}\right)=\max I_{j}\right)$ works the same:

$$
\begin{aligned}
g\left(a_{j}\right)= & g\left(a_{n}\right)+K \cdot d\left(a_{n}, a_{j}\right) \quad n \text { smallest possible } \\
0 \leq f\left(a_{j}\right)-g\left(a_{j}\right)= & f\left(a_{j}\right)-g\left(a_{n}\right)-K \cdot d\left(a_{n}, a_{j}\right) \\
& \mid f\left(a_{j}\right) \leq f\left(a_{n}\right)+K \cdot d\left(a_{n}, a_{j}\right)+\epsilon \\
\leq & f\left(a_{n}\right)-g\left(a_{n}\right)+\epsilon
\end{aligned}
$$

Assume $f\left(a_{n}\right)>g\left(a_{n}\right)$, then $\exists m<n: g\left(a_{n}\right)=g\left(a_{m}\right)+K \cdot d\left(a_{n}, a_{m}\right)$ and

$$
\begin{aligned}
g\left(a_{j}\right) & =g\left(a_{m}\right)+K \cdot\left(d\left(a_{n}, a_{m}\right)+d\left(a_{n}, a_{j}\right)\right) \\
& \geq g\left(a_{m}\right)+K \cdot d\left(a_{m}, a_{j}\right),
\end{aligned}
$$

as $g\left(a_{j}\right) \in I_{j}$, we have equality and thereby

$$
g\left(a_{n}\right)+K \cdot d\left(a_{n}, a_{j}\right)=g\left(a_{m}\right)+K \cdot d\left(a_{m}, a_{j}\right)
$$

which contradicts minimality of $n$.

We now constructed a $K$-Lipschitz function $g$ which is densely defined on $X$. We can easily extend $g$ to $X$, which still is $K$-Lipschitz. Now consider $x \in X$ and let $\left(x_{j}\right) \subseteq\left\{a_{1}, a_{2}, \ldots\right\}$ be a Cauchy sequence with limit $x$. Then we have

$$
\text { and } \begin{aligned}
\left|f(x)-f\left(a_{j}\right)\right| & \leq K \cdot d\left(x, a_{j}\right)+\epsilon \rightarrow \epsilon \\
|g(x)-f(x)| & \leq\left|g(x)-g\left(a_{j}\right)\right|+\left|g\left(a_{j}\right)-f\left(a_{j}\right)\right|+\left|f\left(a_{j}\right)-f(x)\right| \\
& \leq K \cdot d\left(x, a_{j}\right)+\epsilon+K \cdot d\left(x, a_{j}\right)+\epsilon \rightarrow 2 \epsilon
\end{aligned}
$$

Theorem 82

Let $X, Y$ be separable metric spaces, and $\eta: X \rightarrow Y, \xi: Y \rightarrow X$ both $\epsilon$ isometries $(\epsilon \geq 0)$ with $\eta \circ \xi$ and $\xi \circ \eta$ being $2 \epsilon$-near to $\operatorname{id}_{Y}$ respectively $\operatorname{id}_{X}$. Then for each $K \geq 0$ the spaces of $K$-Lipschitz-functions are $6 K \epsilon$-isometric, in particular there exists a $6 \mathrm{~K} \epsilon$-isometry

$$
\bar{\eta}: \operatorname{Lip}_{K}(Y) \rightarrow \operatorname{Lip}_{K}(X)
$$

in respect to the $L^{\infty}$-metric on $\operatorname{Lip}_{K}$, with

$$
d_{\infty}(\bar{\eta}(f), f \circ \eta) \leq 2 K \epsilon
$$

and a corresponding $\bar{\xi}$.

Proof Choose dense countable subsets $\left\{a_{j}\right\} \subseteq X$ and $\left\{b_{j}\right\} \subseteq Y$. For each $f \in \operatorname{Lip}_{K}(Y)$ we have $f \circ \eta: X \rightarrow \mathbb{R}$ which fulfills

$$
d(f \eta x, f \eta y) \leq K d(\eta x, \eta y) \leq K \cdot d(x, y)+K \epsilon .
$$


We smooth this function in the way of Lemma 81 with respect to $\left\{a_{j}\right\}$ (note that $\epsilon$ is replaced by $K \epsilon$ ) and get a $K$-Lipschitz-function which we call

$$
\bar{\eta}(f): X \rightarrow \mathbb{R}
$$

with $d_{\infty}(\bar{\eta}(f), f \circ \eta) \leq 2 K \epsilon$. We now show that the mapping

$$
\bar{\eta}: \operatorname{Lip}_{K}(Y) \rightarrow \operatorname{Lip}_{K}(X)
$$

is a rough isometry with respect to $d_{\infty}$. For this, we first take a look at

$$
d_{\infty}(\bar{\eta}(f), \bar{\eta}(g)) \leq 2 K \epsilon+d_{\infty}(f \circ \eta, g \circ \eta)+2 K \epsilon .
$$

for arbitrary $f, g \in \operatorname{Lip}_{K}(Y)$. It is clear that

$$
\begin{aligned}
d_{\infty}(f \circ \eta, g \circ \eta) & \leq d_{\infty}(f, g) \\
\Rightarrow \quad d_{\infty}(\bar{\eta}(f), \bar{\eta}(g)) & \leq 4 K \epsilon+d_{\infty}(f, g) .
\end{aligned}
$$

On the other hand we have for each $y \in Y$ an $x \in X$ with $d(\eta x, y) \leq \epsilon$, and therefore for all $y \in Y$ :

$$
\begin{aligned}
d(f y, g y) & \leq d(f y, f \eta x)+d(f \eta x, g \eta x)+d(g \eta x, g y) \\
& \leq 2 K \epsilon+d(f \eta x, g \eta x)
\end{aligned}
$$

hence

$$
\begin{aligned}
d_{\infty}(f, g) & \leq 2 K \epsilon+d_{\infty}(f \eta, g \eta) \\
& \leq 6 K \epsilon+d_{\infty}(\bar{\eta}(f), \bar{\eta}(g))
\end{aligned}
$$

which proves the first part of a rough isometry.

In the same way we define $\bar{\xi}: \operatorname{Lip}_{K}(X) \rightarrow \operatorname{Lip}_{K}(Y)$. We now have

$$
\begin{aligned}
d_{\infty}(\bar{\eta}(\bar{\xi} f), f) & \leq d_{\infty}(\bar{\eta}(\bar{\xi} f),[\bar{\xi} f] \circ \eta)+d_{\infty}([\bar{\xi} f] \circ \eta, f \circ \xi \circ \eta) \\
& \leq 2 K \epsilon+2 K \epsilon+2 K \epsilon .
\end{aligned}
$$

Note that for the second term we made use of

$$
d_{\infty}([\bar{\xi} f] \circ \eta, f \circ \xi \circ \eta) \leq d_{\infty}(\bar{\xi} f, f \circ \xi) \leq 2 K \epsilon .
$$

In the same way we approximate $\eta \circ \xi$ and find that they both are $6 K \epsilon$-near to the corresponding identities on $\operatorname{Lip}_{K}(X)$ and $\operatorname{Lip}_{K}(Y)$.

This last step also proves the second property of rough isometries: For $f \in \operatorname{Lip}_{K}(X)$ choose $\bar{\xi}(f)$ and conclude $d(\bar{\eta}(\bar{\xi} f), f) \leq 6 K \epsilon$. 


\subsubsection{Hyperconvex and Non-Hyperconvex Codomains}

Unfortunately, the two previous proofs are only slightly generalizable to other choices for $Z$, in particular for hyperconvex metric + spaces.

Lemma 83

Let $X$ be a separable metric space, $Z$ a hyperconvex metric $c_{+}$space, and $\epsilon, K \geq$ 0 arbitrary. Then $\operatorname{Lip}_{K}(X, Z)$ is $2 \epsilon$-dense in $\operatorname{Lip}_{K, \epsilon}(X, Z)$.

Proof Let $f \in \operatorname{Lip}_{K, \epsilon}(X, Z)$ and $x \in X$ be arbitrary, and choose a countable dense subset $\left\{a_{j}\right\}_{j \in \mathbb{N}^{*}} \subseteq X$. By induction, define $g\left(a_{j}\right)$ as follows: Define $r_{k}:=K \cdot d\left(a_{k}, a_{j}\right)$ and $s_{k}:=r_{k}+\epsilon$ for any $k \in \mathbb{N}^{*}$ and

$$
\mathcal{F}_{j}:=\left\{B_{g\left(a_{k}\right)}\left(r_{k}\right): 1 \leq k<j\right\} \cup\left\{B_{f\left(a_{k}\right)}\left(s_{k}\right): k \in \mathbb{N}^{*}\right\} .
$$

We show that hyperconvexity applies and freely choose $g\left(a_{j}\right) \in \bigcap \mathcal{F}_{j}$ :

1. $d\left(g\left(a_{n}\right), g\left(a_{m}\right)\right) \leq r_{n}+r_{m}$. WLOG assume $n<m$. The value $g\left(a_{m}\right)$ has been defined to fulfill

$$
\begin{aligned}
g\left(a_{m}\right) & \in B_{g\left(a_{n}\right)}\left(K \cdot d\left(a_{n}, a_{m}\right)\right) \\
\Rightarrow \quad d\left(g\left(a_{m}\right), g\left(a_{n}\right)\right) & \leq K \cdot d\left(a_{n}, a_{m}\right) \\
& \leq K \cdot d\left(a_{n}, a_{j}\right)+K \cdot d\left(a_{j}, a_{m}\right)=r_{n}+r_{m} .
\end{aligned}
$$

2. $d\left(f\left(a_{n}\right), g\left(f_{m}\right)\right) \leq s_{n}+s_{m}$. This holds from the fact that $f$ is $(K, \epsilon)$ Lipschitz, plus triangle inequality.

3. $d\left(f\left(a_{n}\right), g\left(a_{m}\right)\right) \leq s_{n}+r_{m}$. Again, $g\left(a_{m}\right)$ has previously been defined to fulfill

$$
\begin{aligned}
g\left(a_{m}\right) & \in B_{f\left(a_{n}\right)}\left(K \cdot d\left(a_{n}, a_{m}\right)+\epsilon\right) \\
\Rightarrow d\left(g\left(a_{m}\right), f\left(a_{n}\right)\right) & \leq K \cdot d\left(a_{n}, a_{m}\right)+\epsilon \\
& \leq K \cdot d\left(a_{n}, a_{j}\right)+K \cdot d\left(a_{j}, a_{m}\right)+\epsilon \\
& =s_{n}+r_{m} .
\end{aligned}
$$

Hyperconvexity yields $\bigcap \mathcal{F}_{j} \neq \emptyset$. Choose $g\left(a_{j}\right) \in \bigcap \mathcal{F}_{j}$ arbitrary.

By definition, $d\left(g\left(a_{j}\right), f\left(a_{j}\right)\right) \leq \epsilon$ and $g$ is $K$-Lipschitz on $\left\{a_{j}\right\}_{j \in \mathbb{N}^{*}}$. We extend $g$ to the whole of $X$ in the same way as in the proof of Lemma 81

We should note that we did not make use of the full strength of hyperconvexity, but restricted to countable families of balls, hence " $\sigma$-hyperconvexity" suffices for Lemma 83] However, as noted by Aronszajn and Panitchpakdi ( $\mathrm{AP}$, Theorem 2.1), $\sigma$-hyperconvexity and separability of $Z$ already imply full hyperconvexity. 
Another possibility to prove Lemma 83 is to show that $\left(\operatorname{Lip}_{K}(X, Z), d_{\infty}\right)$ is hyperconvex and apply the Extension Theorem 2.3 of $\mathrm{AP}$. to the special case

$$
\left(\operatorname{Lip}_{K, \epsilon}(X, Z), d_{\infty}\right) \supseteq\left(\operatorname{Lip}_{K}(X, Z), d_{\infty}\right) \stackrel{\text { id }}{\longrightarrow}\left(\operatorname{Lip}_{K}(X, Z), d_{\infty}\right) .
$$

Note that the preservation of the modulus of continuity of id is of high importance to get a controlled extension. However, showing the hyperconvexity of $\operatorname{Lip}_{K}(X, Z)$ for separable $X$ is again very similar to the proof of Lemma 83. Given a family $f_{k} \in \operatorname{Lip}_{K}(X, Z)$ and non-negative numbers $s_{k}, k \in I$, with $I$ an arbitrary index set, satisfying $d_{\infty}\left(f_{k}, f_{l}\right) \leq s_{k}+s_{l}$ for all $k, l \in K$, we choose a dense subset $\left\{a_{j}\right\}_{j \in J} \subseteq X$ and apply the exact same arguments to the family

$$
\mathcal{F}_{j}:=\left\{B_{g\left(a_{n}\right)}\left(K \cdot d\left(a_{n}, a_{j}\right)\right): 1 \leq n<j\right\} \cup\left\{B_{f_{n}\left(a_{j}\right)}\left(s_{n}\right): n \in I\right\}
$$

with $g\left(a_{j}\right): \in \bigcap \mathcal{F}_{j}$ inductively defined. Finally extending $g$ continuously to the whole of $X$ returns a $K$-Lipschitz function which is within the intersection of all balls $B_{f_{k}}\left(s_{k}\right)$.

In general, the metric + space of continuous real-valued functions $C(H)$ of a compact Hausdorff space $H$ with metric $d_{\infty}$ is not hyperconvex (mostly, it is not even metrically complete) - only in cases where $H$ is extremally disconnected 1 , hyperconvexity can be recovered. In Remark 5.1 of [AP], Aronszajn and Panitchpakdi connect the property of order completeness of the lattice structure of $C(H)$ to hyperconvexity. In view of this argument and of Proposition [43, it is better comprehensible why Lipschitz function spaces are more often hyperconvex than spaces of continuous functions. The situation in the case of non-separable metric + spaces $X$ however is unknown to the author.

Example 84

We analyze an example for a simple non-hyperconvex true metric space, the Euclidean plane $Z=\left(\mathbb{R}^{2}, d_{2}\right)$. Let $L>0$ and $\epsilon>0$ be arbitrary, $X=\{a, b, c\}$ with $d(a, b)=d(b, c)=L, d(a, c)=2 L$, and $f: X \rightarrow \mathbb{R}^{2}$ with

$$
\begin{aligned}
f(a) & =(0,0), \\
f(b) & =\left(L+\frac{1}{2} \epsilon, \sqrt{L \epsilon+\frac{3}{4} \epsilon^{2}}\right), \\
\text { and } f(c) & =(2 L+\epsilon, 0) .
\end{aligned}
$$

We find $f \in \operatorname{Lip}_{1, \epsilon}(X, Z)$. To smooth $f$ like in the proof of Lemma 81, we choose $a_{1}=a, a_{2}=c, a_{3}=b$. Hence, $g(a)=f(a)=(0,0)$. As

$$
2 L<|g(a)-g(c)| \leq 2 L+\epsilon,
$$

we choose $g(c)$ to be the point in the $2 L$-ball around $g(a)$ nearest to $f(c)$, this is $g(c)=(2 L, 0)$. Now, there is only one possibility left for $g(b)$ to make $g$ Lipschitz, we have to set $g(b)=(L, 0)$. However,

$$
d(g(b), f(b))=\sqrt{L \epsilon+\epsilon^{2}},
$$

\footnotetext{
${ }^{1}$ i.e. the closure of each open set is open.
} 
which can be chosen arbitrarily large for fixed $\epsilon$. Subsequently, the algorithm and proof fail.

The proof's algorithm of the more general Lemma 83 fails as well, in step $j=3$ the intersection of the five involved balls is empty, reflecting the nonhyperconvexity of the Euclidean plane.

However, in this special case $\left(Z=\mathbb{R}^{2}\right.$ Euclidean, $X$ is a true metric space of three points), the thesis of the two Lemmas still holds, one only has to choose the order of the points $a_{j}$ more carefully: $a_{1}=b, a_{2}=a, a_{3}=c$ will yield

$$
d\left(g\left(a_{2}\right), g\left(a_{3}\right)\right)=\frac{2 L^{2}+L \epsilon}{L+\epsilon}<2 L,
$$

thus $g \in \operatorname{Lip}(X, Z), d_{\infty}(f, g)=\epsilon$, and $\operatorname{Lip}(X, Z)$ is $\epsilon$-dense in $\operatorname{Lip}_{1, \epsilon}(X, Z)$.

Example 85 85

In other examples, topological obstructions prevent the smoothening. Let $X=$ $S^{1}$ with arc length metric and of total length $2 \pi, Z=S^{1}$ also with arc length metric, but total length $2 \pi+2 \epsilon, \epsilon>0$. The identity $f=$ id $: X \rightarrow Z$ is $(1, \epsilon)$-Lipschitz. However, there cannot be a Lipschitz function $g: X \rightarrow Z$ with $d_{\infty}(f, g)=O(\epsilon)$, only $O(\sqrt{\epsilon})$.

Example 86

Take the unit sphere $Z=S^{2}$ with arc length metric, and $X=S^{1}$ with arc length metric and of total length $2 \pi-\epsilon$. Let $f$ be the embedding of $X$ as the equator of $Z$, then $f$ is $(1, \epsilon)$-Lipschitz. Although there are Lipschitz-embeddings of $X$ into $Z$, their distance to $f$ is proportional to $\sqrt{\epsilon}$, which inhibits a result in the sense of Lemma 81. As this argument still holds after removal of a part of the sphere near one of the poles, we conclude that there is even a topologically contractible space $Z$ for which the thesis of Lemma 81 fails.

Example 87

Let $G=\langle S\rangle$ be a finitely generated group, which acts freely and cocompactly on a Riemannian manifold $M$. Let $x_{0} \in M$ be arbitrary, then $G x_{0}$ is an $\epsilon$-dense subset of $M$ for some large enough $\delta>0$. Define $f: M \rightarrow[0, \infty)$ by

$$
x \mapsto \quad \min _{g \in G:} d_{S}(e, g),
$$

i.e. wordlength of (one of) the nearest elements out of $G x_{0}$. This defines a $(K, \epsilon)$-Lipschitz function for large enough $K, \epsilon>0$, and hence a K-Lipschitz function $\bar{f}: M \rightarrow[0, \infty)$, which represents the wordlength of $G$ in $M$, at least up to some error $2 \epsilon$.

If for example $M$ is the Euclidean plane, $G=\mathbb{Z}^{2}$ with standard action and standard generators $S$, then we may choose $\delta=1$ and will find $f$ to be $(K=\sqrt{2}, \epsilon=2)$-Lipschitz, and $g$ will be a $\sqrt{2}$-Lipschitz function near to the supremum norm, depending on the chosen dense sequence $\left(a_{j}\right) \subseteq \mathbb{R}^{2}$. 


\subsubsection{Algorithmic Aspects}

The proof used for Lemma 81 is an online algorithm, i.e. it is possible to start the calculation of the smoothed function $g$ without having all information about $f$ available. Even better, to calculate the next value $g\left(a_{j}\right)$ the only information you need is minimal: The previously calculated values of $g$, the distance of $a_{j}$ to all previously used points, and $f\left(a_{j}\right)$ itself. In addition, you are free to choose the next point $a_{j+1}$ freely, without risking inconsistencies. This is of importance to efficient approximations of $g(a)$ with $a=\lim _{j \rightarrow \infty} a_{j}$, as well as to any successive algorithm that needs Lipschitz functions as input to return a realistic result, such as deconvolution filters.

Lemma 83 is superior to Lemma 81 in its conclusion, but its proof does not exhibit the same qualities. Apart from the general need for the Countable Axiom of Choice and the need to handle infinite intersections, it is also necessary to provide all information about $f$ already to calculate $g\left(a_{2}\right)$.

Example 84 finally provides a glimpse at the worst case scenario: Here, the choice of an admissible sequence of the points $\left(a_{j}\right)$ was necessary to gain a reasonable smoothening. While our example employs a finite true metric space $X$, it is obvious to conjecture that infinite or even uncountable true metric spaces $X$ might require an infinite automaton to return an admissible sequence based on a given function $f$. The consequence is that at least some of the values of $g$ would be non-computable numbers in the sense of $[\mathbb{T}$ (in $Z$ instead of $\mathbb{R}$ ). In this scenario, the main interesting question, namely whether $\operatorname{Lip}_{K}(X, Z)$ is $O(\epsilon)$-dense in $\operatorname{Lip}_{K, \epsilon}(X, Z)$ or not, could even be undecidable.

\subsection{Rough ml-Isomorphisms}

Next we define a special version of rough isometry, suiting the lattice structure of a complete lattice with intervaluation metric ${ }_{+}$in general, and of a Lipschitz function space in particular. The main new property will be an "approximate lattice homomorphism". In the Lipschitz-function case, it exists in various versions, as Thomas Schick pointed out to us:

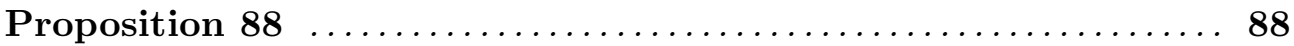

Let $X, Y$ be metric spaces and let $\kappa: \operatorname{Lip} Y \rightarrow \operatorname{Lip} X$ be an $\epsilon$-isometric embedding, $\epsilon \geq 0$ arbitrary and fixed. Then the following properties are equivalent:

1. $f \leq g \Rightarrow \kappa f \leq \kappa g+\epsilon$ for all $f, g \in \operatorname{Lip} Y$

2. $d_{\infty}((\kappa f) \vee(\kappa g), \kappa(f \vee g)) \leq \epsilon$ for all $f, g \in \operatorname{Lip} Y$

3. For all $f_{j} \in \operatorname{Lip} Y, j \in J, J \neq \emptyset$ some index set, holds:

$$
d_{\infty}\left(\bigvee_{j \in J} \kappa f_{j}, \kappa \bigvee_{j \in J} f_{j}\right) \leq \epsilon \text { and } d_{\infty}\left(\bigwedge_{j \in J} \kappa f_{j}, \kappa \bigwedge_{j \in J} f_{j}\right) \leq \epsilon
$$


Proof $(3) \Rightarrow(2): \# J=2$.

$(2) \Rightarrow(\mathbf{1}):$ Assume there is some $x \in X$ such that $(\kappa f)(x)>(\kappa g)(x)+\epsilon$. From $f \leq g$ follows $f \vee g=g$, thus $d_{\infty}((\kappa f) \vee(\kappa g), \kappa g) \leq \epsilon$, in particular $(\kappa f)(x) \vee(\kappa g)(x) \leq(\kappa g)(x)+\epsilon$, contradiction.

(1) $\Rightarrow$ (3): Obviously we have $f_{k} \leq \bigvee_{j \in J} f_{j}$ for all $k \in J$, hence $\kappa f_{k} \leq \kappa \bigvee_{j} f_{j}+$ $\epsilon$. We calculate the supremum over all $k \in J: \bigvee_{j} \kappa f_{j} \leq \kappa \bigvee_{j} f_{j}+\epsilon$. On the other hand, for every $\delta>0$ there is some $k \in J$ with $d_{\infty}\left(f_{k}, \bigvee_{j} f_{j}\right) \leq \delta$. As $\kappa$ is an $\epsilon$-isometric embedding, this yields $d_{\infty}\left(\kappa f_{k}, \kappa \bigvee_{j} f_{j}\right) \leq \delta+\epsilon$, hence

$$
\kappa \bigvee_{j \in J} f_{j} \leq \kappa f_{k}+\epsilon+\delta \leq \bigvee_{j \in J} \kappa f_{j}+\epsilon+\delta
$$

The limit $\delta \rightarrow 0$ yields the first approximation, the other works analogously.

We extend property (3) from Proposition 88 to allow $J=\emptyset$, generalize to arbitrary intervaluation metrics, and use it to define the notion of ml-isomorphisms:

Definition 89 89

Let $L, L^{\prime}$ be intervaluation metric lattices. An $\epsilon$-ml-homomorphism, $\epsilon \geq 0$ is an $\epsilon$-isometric embedding $\kappa: L \rightarrow L^{\prime}$, with

$$
d_{\infty}\left(\bigvee_{j \in J} \kappa f_{j}, \kappa \bigvee_{j \in J} f_{j}\right) \leq \epsilon \text { and } d_{\infty}\left(\bigwedge_{j \in J} \kappa f_{j}, \kappa \bigwedge_{j \in J} f_{j}\right) \leq \epsilon
$$

for all $f_{j} \in L, j \in J, J$ some arbitrary index set.

An $\epsilon$-ml-isomorphism is a pair of $\epsilon$-ml-homomorphisms $\kappa: L \rightarrow L^{\prime}$ and $\kappa^{\prime}$ : $L^{\prime} \rightarrow L$, such that $\kappa \circ \kappa^{\prime}$ and $\kappa^{\prime} \circ \kappa$ are $\epsilon$-near their corresponding identities. When we speak of an " $\epsilon$-ml-isomorphism $\kappa$ ", the corresponding $\kappa^{\prime}$ shall always be implied.

Proposition 90

Let $L$ be complete, and let 0 be the least element in $L$. For any $\epsilon$-ml-isomorphism $\kappa$ holds $d(\kappa(0), 0) \leq \epsilon$.

Proof As Andreas Thom pointed out, this follows directly from Definition 89 when $J=\emptyset$. There is also a $3 \epsilon$-proof avoiding empty index sets: Let $\kappa: L \rightarrow L^{\prime}$ be an $\epsilon$-ml-isomorphism. We certainly know $0 \wedge \kappa^{\prime}(0)=0 \in L$, hence

$$
d\left(\kappa(0), \kappa(0) \wedge \kappa \kappa^{\prime}(0)\right) \leq 2 \epsilon .
$$

Now apply the cut law (see Definition 62, Corollary [71) to see

$$
d\left(\kappa(0) \wedge \kappa \kappa^{\prime}(0), \kappa(0) \wedge 0\right) \leq \epsilon
$$

and use $\kappa(0) \wedge 0=0 \in L$. 
Proposition 91 .................................... 91

A $\delta$-surjective $\epsilon$-ml-homomorphism $\kappa: \operatorname{Lip} Y \rightarrow \operatorname{Lip} X$ induces $a(2 \epsilon+2 \delta)$-mlisomorphism $\left(\kappa, \kappa^{\prime}\right)$.

Proof For each $f \in \operatorname{Lip} X$ choose an element $\kappa^{\prime}(f)$, such that $d\left(\kappa \kappa^{\prime} f, f\right) \leq \delta$. We show that the pair $\left(\kappa, \kappa^{\prime}\right)$ defines a $(2 \epsilon+2 \delta)$-ml-isomorphism. The first inequality in Definition 89 is standard in coarse geometry:

$$
\begin{aligned}
& d_{\infty}(f, g) \leq d_{\infty}\left(\kappa \kappa^{\prime} f, \kappa \kappa^{\prime} g\right)+2 \delta \leq d_{\infty}\left(\kappa^{\prime} f, \kappa^{\prime} g\right)+(\epsilon+2 \delta) \\
& d_{\infty}(f, g) \geq d_{\infty}\left(\kappa \kappa^{\prime} f, \kappa \kappa^{\prime} g\right)-2 \delta \geq d_{\infty}\left(\kappa^{\prime} f, \kappa^{\prime} g\right)-(\epsilon+2 \delta)
\end{aligned}
$$

for all $f, g \in \operatorname{Lip} X$. We now show that $\kappa^{\prime}$ fulfills the second and third inequality as well. Both can be handled the same way:

$$
\begin{aligned}
d_{\infty}\left(\bigwedge \kappa^{\prime} f_{j}, \kappa^{\prime} \bigwedge f_{j}\right) & \leq d_{\infty}\left(\kappa \bigwedge \kappa^{\prime} f_{j}, \kappa \kappa^{\prime} \bigwedge f_{j}\right)+\epsilon \\
& \leq d_{\infty}\left(\kappa \bigwedge \kappa^{\prime} f_{j}, \bigwedge f_{j}\right)+\epsilon+\delta \\
& \leq d_{\infty}\left(\bigwedge \kappa \kappa^{\prime} f_{j}, \bigwedge f_{j}\right)+2 \epsilon+\delta \\
& \leq \bigvee d_{\infty}\left(\kappa \kappa^{\prime} f_{j}, f_{j}\right)+2 \epsilon+\delta \\
& \leq \delta+2 \epsilon+\delta \quad \forall f_{j} \in \operatorname{Lip} X, j \in J
\end{aligned}
$$

Here we used (i) $\kappa$ is $\epsilon$-isometric embedding, (ii) $\kappa \kappa^{\prime}$ is near identity, (iii) $\kappa$ is ml-homomorphism, (iv) Proposition 72 (v) $\kappa \kappa^{\prime}$ is near identity.

Finally we show that $\kappa^{\prime} \kappa$ is $(\epsilon+\delta)$-near identity:

$$
d_{\infty}\left(\kappa^{\prime} \kappa f, f\right) \leq d_{\infty}\left(\kappa \kappa^{\prime}(\kappa f),(\kappa f)\right)+\epsilon \leq \delta+\epsilon \quad \forall f \in \operatorname{Lip} X .
$$

Lemma 92

Let $L$ and $L^{\prime}$ be intervaluation metric $c_{+}$lattices with least elements, and let the family of all bounded completely ml-irreducible elements bcmli $\left(L^{\prime}\right)$ be a base of $L^{\prime}$ (see Definitions 73 and 76). Then any $\epsilon$-ml-isomorphism $\kappa: L \rightarrow L^{\prime}, \epsilon \geq 0$ maps $\operatorname{bcmli}(L) 6 \epsilon$-near $\operatorname{bcmli}\left(L^{\prime}\right)$.

Proof Let $p \in \operatorname{bcmli}(L)$ be some bounded completely ml-irreducible element. Represent $\kappa(p)$ via elements $q_{j} \in \operatorname{bcmli}\left(L^{\prime}\right), j \in J, J$ some non-empty index set. Let $\delta>0$ be arbitrary. Then we have

$$
\begin{aligned}
d_{\infty}\left(\kappa(p), \bigvee q_{j}\right) & =0 \quad \mid \text { apply } \kappa^{\prime} \\
\Rightarrow \quad d_{\infty}\left(p, \bigvee \kappa^{\prime}\left(q_{j}\right)\right) & \leq 3 \epsilon .
\end{aligned}
$$

As $p$ is completely ml-irreducible, we know that there exists $k \in J$ such that

$$
\begin{array}{rlrl}
d_{\infty}\left(p, \kappa^{\prime}\left(q_{k}\right)\right) & \leq 3 \epsilon+\delta & \mid \text { apply } \kappa \\
\Rightarrow \quad d_{\infty}\left(\kappa(p), q_{k}\right) & \leq 5 \epsilon+\delta .
\end{array}
$$


Case 1: $\epsilon>0$. Choose $\delta=\epsilon$.

Case 2: $\epsilon=0$. The preceding argument yields a sequence of bounded completely ml-irreducible elements $q_{k}$ metrically converging to $\kappa(p)$. As of Proposition 75 , $\kappa(p)$ must be bounded and completely ml-irreducible as well.

\section{$2.3 \quad \Lambda$-Functions}

Lip $X$ is no algebra, like e.g. $C(X)$. Thus we cannot give a linear base of functions and reconstruct $\operatorname{Lip} X$ by linear combinations. However, we can use the lattice structure to give a base in the sense of Definition $[76$ for $\operatorname{Lip} X$ : Minimal Lipschitz functions with a given value at a single point.

Definition 93

Let $x, y \in X$ and $r \in[0, \infty]$ be arbitrary. Define $\Lambda(x, r) \in \operatorname{Lip} X$ by

$$
\Lambda(x, r)(y):=(r-d(x, y)) \vee 0 .
$$

Note that this definition applies to $r=\infty$ or $d(x, y)=\infty$ as well: If $d(x, y)=\infty$, we have $\Lambda(x, r)(y)=0$, and if $r=\infty$ :

$$
\Lambda(x, \infty)(y)=\left\{\begin{array}{lll}
\infty & : & d(x, y) \neq \infty \\
0 & : & d(x, y)=\infty
\end{array}\right.
$$

$\Lambda$-functions with $r=\infty$ will be called infinite, else bounded. Infinite $\Lambda$-functions are infinitely high characteristic functions for $X$ 's components.

Proposition 94

Consider $x, y \in X$ and $r, s \in[0, \infty]$. Then holds:

$$
\begin{aligned}
d_{\infty}(\Lambda(x, r), \Lambda(y, s)) & = \begin{cases}r \vee s & : d(x, y) \geq r \wedge s \\
|r-s|+d(x, y) & : d(x, y) \leq r \wedge s<\infty \\
0 & : d(x, y)<r \wedge s=\infty\end{cases} \\
\leq|r-s|+d(x, y) &
\end{aligned}
$$

Proof Note that if $d(x, y)=r \wedge s$ the first and second case coincide, as $|r-s|=(r \vee s)-(r \wedge s)$. Assume without restriction $r \leq s$. Consider

$$
\begin{aligned}
f z & :=|(0 \vee(r-d(x, z)))-(0 \vee(s-d(y, z)))| \quad \forall z \in X \\
d & :=d_{\infty}(\Lambda(x, r), \Lambda(y, s))=\bigvee_{z \in X} f(z) .
\end{aligned}
$$

Let us start with the infinite cases. If $r=s=d(x, y)=\infty$, we get $d=\infty$ on both sides. If $r=s=\infty, d(x, y) \neq \infty$, we get $d=0$. This is correct, as in this case the $\Lambda$-functions are equal. If $s=\infty, r \neq \infty$ we get $d=\infty$ again, 


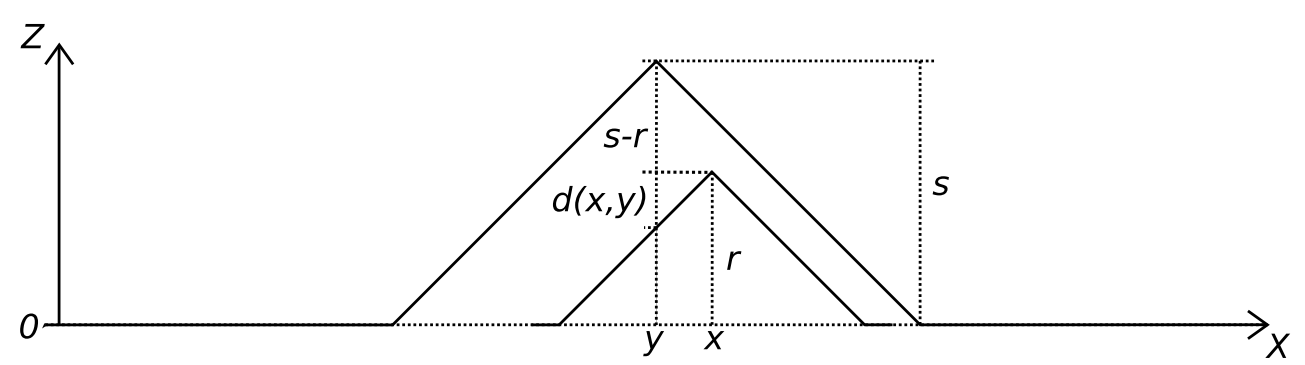

Figure 2.1: The $d_{\infty}$-distance between two $\Lambda$-functions is determined by their difference evaluated at the maximum point of the greater function, see Prop. 94

for each variant of $d(x, y)$. If $r, s \neq \infty$ but $d(x, y)=\infty$, the two $\Lambda$-functions have different components as support, and thus $d$ becomes the maximum of the differences, this is $s$.

Now we assume $r, s, d(x, y) \neq \infty$. First case: $r \leq d(x, y)$. Then we have

$$
d \geq f y=|s-(0 \vee(r-d(x, y)))|=s .
$$

In addition, we have $\Lambda(x, r)(z) \in[0, r]$ and $\Lambda(y, s)(z) \in[0, s]$, thus $f z \leq r \vee s=s$, hence $d=s$. Second case: $d(x, y) \leq r$. We find

$$
d \geq f y=|s-(0 \vee(r-d(x, y)))|=s-r+d(x, y),
$$

and finally:

$$
\begin{aligned}
f z & =|r-d(x, z)-s+d(y, z)| \leq|r-s|+|d(y, z)-d(x, z)| \\
& \leq|r-s|+d(x, y)=s-r+d(x, y) \quad \forall z \in X
\end{aligned}
$$

Corollary 95

For all $x, y \in X$ holds:

$$
d(x, y)=\lim _{r \rightarrow \infty, r \neq \infty} d_{\infty}(\Lambda(x, r), \Lambda(y, r))
$$

Proof Follows directly from Proposition 94

This Corollary points us at an interesting aspect of $\Lambda$-functions: When we analyze the metric + space $X_{r}:=\{\Lambda(x, r): x \in X\}$ with metric $d_{\infty}$ for a fixed $r \in \mathbb{R}_{>0}$, we find it to be naturally isometric to $\left(X, d_{r}\right)$ with the cut-off-metric $d_{r}(x, y):=r \wedge d(x, y)$ for all $x, y \in X$. Only in the limit $r \rightarrow \infty, d_{\infty}$ will restore the full metric of $X$. Ironically, $d_{\infty}$ obviously cuts away the coarse, largescale information of $X$ (in which we are primarily interested) and preserves the topological, small-scale information. The large-scale information of $X$ is still present, but more subtle to access. 


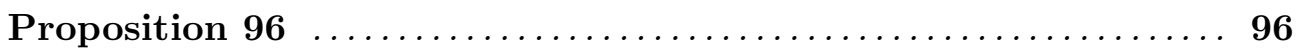

For all $f \in \operatorname{Lip} X$ holds: $f=\bigvee_{x \in X} \Lambda(x, f(x))$, where the latter is a pointwise maximum, not only supremum (cp. Figure 2.2). If $X$ is complete, the set $A:=\{\Lambda(x, f(x)): x \in X\} \cup\{0\}$ is (topologically) closed.

Proof Define $g:=\bigvee_{x \in X} \Lambda(x, f x)$. Clearly, we have $\forall z \in X: f z \leq g z$, as $f z=\Lambda(z, f z)(z)$. We now observe that

$$
f z \geq \Lambda(x, f x)(z) \quad \forall x, z \in X .
$$

For $d(x, z) \geq f x$, this is clear. For $d(x, z) \leq f x$, this follows from Lipschitz continuity $(f z \geq f x-d(x, z))$.

Furthermore, we notice that we deal with pointwise maxima: Each supremum of $\{\Lambda(x, f x)(z)\}_{x \in X}$ is taken by $\Lambda(z, f z)(z)=f z$.

Let $\Lambda\left(x_{j}, f x_{j}\right)$ be any sequence converging to $g \in \operatorname{Lip} X, x_{j} \in X, j \in \mathbb{N}^{*}$. First we notice

$$
\begin{aligned}
f x_{j}=d_{\infty}\left(0, \Lambda\left(x_{j}, f x_{j}\right)\right) & \geq d_{\infty}(0, g)-d_{\infty}\left(g, \Lambda\left(x_{j}, f x_{j}\right)\right) \\
\text { and } f x_{j} & \leq d_{\infty}(0, g)+d_{\infty}\left(g, \Lambda\left(x_{j}, f x_{j}\right)\right),
\end{aligned}
$$

hence $f x_{j} \rightarrow d_{\infty}(0, g)$. Assume $g \neq 0$ and finite. Then there is $x \in X$ with $g x>0$ and $f x_{j}$ must have a lower bound $R>0$ for large enough $j$. By Cauchy criterion there is $N \in \mathbb{N}^{*}$ such that for all $j, k>N$ we have

$$
d_{\infty}\left(\Lambda\left(x_{j}, f x_{j}\right), \Lambda\left(x_{k}, f x_{k}\right)\right) \leq \frac{1}{2} R<f x_{j} \wedge f x_{k} .
$$

Due to Proposition 94 we conclude that for large enough $j, k$

$$
d_{\infty}\left(\Lambda\left(x_{j}, f x_{j}\right), \Lambda\left(x_{k}, f x_{k}\right)\right)=\left|f x_{j}-f x_{k}\right|+d\left(x_{j}, x_{k}\right) \rightarrow 0 .
$$

Thus $f x_{j}$ as well as $x_{j}$ are Cauchy-sequences. As $X$ and $[0, \infty]$ are metrically complete, we find $x^{\prime}:=\lim x_{j}$. As $f$ is continuous, we have $f x^{\prime}=\lim f x_{j}$, and $\Lambda\left(x^{\prime}, f x^{\prime}\right) \in A$. Now we only have to show $g=\Lambda\left(x^{\prime}, f x^{\prime}\right)$. But this is clear, as for large enough $j$ we have

$$
d_{\infty}\left(\Lambda\left(x^{\prime}, f x^{\prime}\right), \Lambda\left(x_{j}, f x_{j}\right)\right) \leq\left|f x^{\prime}-f x_{j}\right|+d\left(x^{\prime}, x_{j}\right) \rightarrow 0+0 .
$$

Now assume $g$ to be infinite (i.e. $\exists x: g x=\infty$ ). Then $f x_{j}$ has to be infinite as well for large enough $j$ (there is no non-trivial convergence to infinity in the chosen metric on $[0, \infty)$ and Proposition 94 shows $d\left(x_{j}, x_{k}\right)<\infty$ for large enough $j, k)$. Hence $\Lambda\left(x_{j}, f x_{j}\right)=\Lambda\left(x_{k}, f x_{k}\right)=g$.

Corollary 97

The family of all bounded $\Lambda$-functions is a metrically closed base in the sense of Definition 76 . 


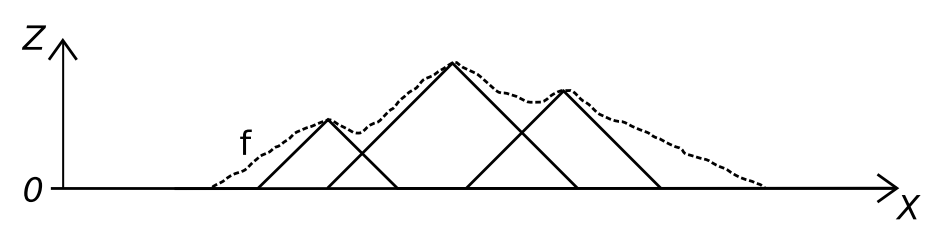

Figure 2.2: Proposition 96 demonstrates how to decompose arbitrary 1Lipschitz functions into a supremum of $\Lambda$-functions.

Proof We note that $\Lambda(x, \infty)$ is the supremum of all $\Lambda(x, r)$ with $r \in[0, \infty)$, i.e. we do not need infinite $\Lambda$-functions to represent functions from $\operatorname{Lip} X$. In addition, the family of all bounded $\Lambda$-functions is an intersection of the closed set of all $\Lambda$-functions and the closed component of the zero-function, thus it is closed as well.

We make some more use of the black magic of Proposition 72 .

Proposition 98

For all $\epsilon$-isometries $\eta: X \rightarrow Y$ and $f \in \operatorname{Lip} Y$ holds:

$$
d_{\infty}\left(\bigvee_{x \in X} \Lambda(x, f \eta x), \bigvee_{y \in Y} \Lambda\left(\eta^{\prime} y, f y\right)\right) \leq \epsilon
$$

Proof We observe that $d:=d_{\infty}\left(\bigvee_{x \in X} \Lambda(x, f \eta x), \bigvee_{y \in Y} \Lambda\left(\eta^{\prime} y, f y\right)\right)$ can be rewritten to

$$
d=d_{\infty}\left(\bigvee_{(x, y) \in J} \Lambda(x, f \eta x), \bigvee_{(x, y) \in J} \Lambda\left(\eta^{\prime} y, f y\right)\right)
$$

where $J:=\left\{(x, y) \in X \times Y: y=\eta x\right.$ or $\left.x=\eta^{\prime} y\right\}$ : Each element of $X$ (respectively $Y$ ) appears at least once in $J$, and multiple instances do not matter, as $\checkmark$ is idempotent. Now Proposition 72 yields:

$$
d \leq \bigvee_{(x, y) \in J} d_{\infty}\left(\Lambda(x, f \eta x), \Lambda\left(\eta^{\prime} y, f y\right)\right)
$$

Let $(x, y) \in J$. Case 1: $y=\eta x$. Then

$$
\begin{aligned}
d_{\infty}\left(\Lambda(x, f \eta x), \Lambda\left(\eta^{\prime} y, f y\right)\right) & =d_{\infty}\left(\Lambda(x, f \eta x), \Lambda\left(\eta^{\prime} \eta x, f \eta x\right)\right) \\
& \leq d\left(x, \eta^{\prime} \eta x\right) \leq \epsilon
\end{aligned}
$$

Case 2: $x=\eta^{\prime} y$ :

$$
\begin{aligned}
d_{\infty}\left(\Lambda(x, f \eta x), \Lambda\left(\eta^{\prime} y, f y\right)\right) & =d_{\infty}\left(\Lambda\left(\eta^{\prime} y, f \eta \eta^{\prime} y\right), \Lambda\left(\eta^{\prime} y, f y\right)\right) \\
& \leq\left|f \eta \eta^{\prime} y-f y\right| \leq \epsilon
\end{aligned}
$$




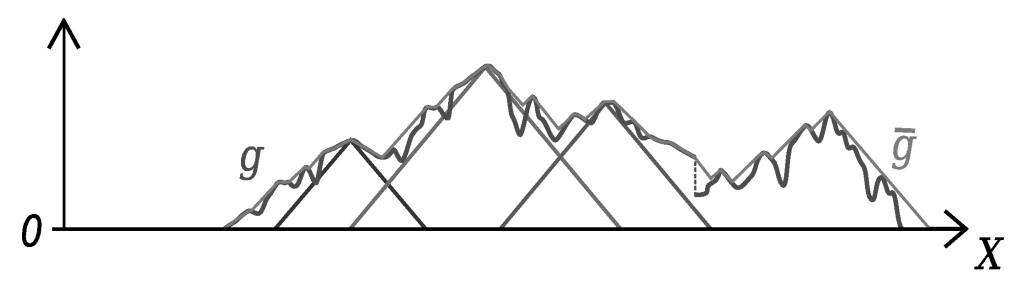

Figure 2.3: "Lipschitzization" $\bar{g}$ of a roughly Lipschitz function $g$ (see Lemma 991). Three of the composing $\Lambda$-functions are shown.

We make a first use of the notions we derived. We will first show a smoothening theorem in the manner of Section 2.1, but using $\Lambda$-functions as new tools. We will then lift each $\epsilon$-isometry $\eta: X \rightarrow Y$ to an $\epsilon$-isometry $\bar{\eta}: \operatorname{Lip} Y \rightarrow \operatorname{Lip} X$. Even better, $\bar{\eta}$ is an $\epsilon$-ml-isomorphism, and is near $f \mapsto f \circ \eta$.

Lemma 99

Let $f \in \operatorname{Lip}_{1, \epsilon}(X,[0, \infty])$. Define

$$
\bar{f}:=\bigvee_{x \in X} \Lambda(x, f x)
$$

Then $f$ and $\bar{f}$ are $\epsilon$-near (cp. Figure 2.3).

Proof We observe that $f(y)$ is never larger than $\bigvee_{x \in X} \Lambda(x, f x)(y)$ for all $y \in X$. So we have

$$
d_{\infty}\left(f, \bigvee_{x \in X} \Lambda(x, f x)\right)=\bigvee_{x, y \in X}(\Lambda(x, f x)(y)-f(y))
$$

and furthermore

$$
\Lambda(x, f x)(y)-f(y)=\left\{\begin{array}{ll}
-f(y) & : d(x, y) \geq f(x) \\
f(x)-f(y)-d(x, y) & : d(x, y) \leq f(x)
\end{array} .\right.
$$

As $f(x)-f(y)-d(x, y) \leq \epsilon$ and $-f(y) \leq 0 \leq \epsilon$ we conclude the statement. (Note that each negative value is surpassed by at least one non-negative value, i.e. $-f(y)$ never occurs after taking the supremum.)

Proposition 100

If $\eta: X \rightarrow Y$ is an $\epsilon$-isometry, and $\kappa: \operatorname{Lip} Y \rightarrow \operatorname{Lip} X$ any mapping which is $\delta$-near $f \mapsto f \circ \eta$, then $\kappa$ is a $(2 \epsilon+2 \delta)$-ml-homomorphism.

Proof (i) We show $\left|d_{\infty}(f, g)-d_{\infty}(\kappa f, \kappa g)\right| \leq 2 \epsilon+2 \delta$ for all $f, g \in \operatorname{Lip} X$. We have

$$
\left|d_{\infty}(\kappa f, \kappa g)-d_{\infty}(f \circ \eta, g \circ \eta)\right| \leq 2 \delta .
$$


As next we notice $d_{\infty}(f \circ \eta, g \circ \eta) \leq d_{\infty}(f, g)$. Now let $y \in Y$ be arbitrary, $x:=\eta^{\prime} y \in X$. Then $|f \eta x-f y| \leq \epsilon$ as $f$ is 1-Lipschitz. Hence

$$
\begin{aligned}
|f y-g y| & \leq|f \eta x-g \eta x|+2 \epsilon \leq d_{\infty}(f \circ \eta, g \circ \eta)+2 \epsilon \\
\Rightarrow \quad d_{\infty}(f, g) & \leq d_{\infty}(f \circ \eta, g \circ \eta)+2 \epsilon .
\end{aligned}
$$

(ii) For $J=\emptyset$ we observe that $d_{\infty}(\kappa(0), 0 \circ \eta) \leq \delta$ and $0 \circ \eta=0$, as well as $d_{\infty}(\kappa(\infty), \infty \circ \eta) \leq \delta$ and $\infty \circ \eta=\infty$. Hence, assume $J \neq \emptyset$. We know

$$
d_{\infty}\left(\kappa\left(\bigwedge f_{j}\right), \bigwedge\left(f_{j} \circ \eta\right)\right)=d_{\infty}\left(\kappa\left(\bigwedge f_{j}\right),\left(\bigwedge f_{j}\right) \circ \eta\right) \leq \delta
$$

as the infimum is calculated pointwise. Hence, with Proposition 72

$$
\begin{aligned}
d_{\infty}\left(\bigwedge\left(\kappa f_{j}\right), \kappa\left(\bigwedge f_{j}\right)\right) & \leq d_{\infty}\left(\bigwedge\left(\kappa f_{j}\right), \bigwedge\left(f_{j} \circ \eta\right)\right)+\delta \\
& \leq \bigvee d_{\infty}\left(\kappa f_{j}, f_{j} \circ \eta\right)+\delta \\
& \leq \epsilon+\delta .
\end{aligned}
$$

Same for supremum.

Theorem 101 (= Th. 21)

Given an $\epsilon$-isometry $\eta: X \rightarrow Y, \bar{\eta}(f):=\overline{f \circ \eta}$ defines a 4t-ml-isomorphism from $\operatorname{Lip} Y$ to $\operatorname{Lip} X$.

Proof Let $f \in \operatorname{Lip} Y$ be arbitrary. $f \circ \eta$ satisfies

$$
d(f \eta x, f \eta y) \leq d(\eta x, \eta y) \leq d(x, y)+\epsilon .
$$

Hence, $f \circ \eta$ and $\bar{\eta}(f)$ are $\epsilon$-near (Lemma 99). However, $\bar{\eta}(f)$ is in Lip $X$, as it is a supremum of Lipschitz functions. Thus we can apply Proposition 100 to $\bar{\eta}: \operatorname{Lip} Y \rightarrow \operatorname{Lip} X$. Same holds for $\eta^{\prime}$ (Definition 31). It remains to show that $\bar{\eta} \circ \overline{\eta^{\prime}}$ and $\overline{\eta^{\prime}} \circ \bar{\eta}$ are near their respective identities.

We already saw that $\bar{\eta}\left(\overline{\eta^{\prime}}(f)\right)$ is $\epsilon$-near $\left(\overline{\eta^{\prime}} f\right) \circ \eta$. Similarly $\overline{\eta^{\prime}} f$ is $\epsilon$-near $f \circ \eta^{\prime}$ and thus $\left(\overline{\eta^{\prime}} f\right) \circ \eta$ is $\epsilon$-near $f \circ \eta^{\prime} \circ \eta$. Finally, $\eta^{\prime} \circ \eta$ is $\epsilon$-near identity, and as $f$ is 1-Lipschitz, $f \circ \eta^{\prime} \circ \eta$ is $\epsilon$-near $f$, too. All this adds up to $3 \epsilon$. Same for $\overline{\eta^{\prime}} \circ \bar{\eta}$.

\section{$2.4 \Lambda$-Functions are Completely ml-Irreducible}

Lemma 102

Consider $p \in \operatorname{Lip} Y$ with $Y$ complete. Then the following are equivalent:

1. $p$ is a bounded $\Lambda$-function, i.e. $\exists x \in Y, r \in[0, \infty): p=\Lambda(x, r)$,

2. $p$ is a bounded completely ml-irreducible element (see Definition 73). 
Proof For convenience, we repeat the definition of a completely ml-irreducible element $p$ : For all $\left(f_{j}\right)_{j \in J} \subseteq \operatorname{Lip} Y$ with an arbitrary, non-empty index set $J$, and any $R \in[0, \infty]$ holds:

$$
d_{\infty}\left(p, \bigvee_{j \in J} f_{j}\right) \leq R \Rightarrow \forall \delta>0 \exists j \in J: d_{\infty}\left(p, f_{j}\right) \leq R+\delta
$$

The case $R=\infty$ is trivial. Hence, assume $R$ to be finite.

(1) $\Rightarrow(\mathbf{2})$ : Let $\left(f_{j}\right)_{j \in J} \subseteq \operatorname{Lip} Y$ and $R \geq 0$ be such that $d_{\infty}\left(p, \bigvee_{j \in J} f_{j}\right) \leq R$ holds. Choose $\delta>0$ arbitrary and $p=\Lambda(y, s)$, for some $y \in Y, s \in[0, \infty)$. As

$$
d\left(p(y), \bigvee f_{j}(y)\right) \leq R \Rightarrow p(y)-R-\delta<\bigvee f_{j}(y)
$$

there has to be a $k \in J$ such that $p(y)-R-\delta<f_{k}(y)$, otherwise $p(y)-R-\delta$ would be a smaller upper bound for all $f_{j}$ then $\bigvee f_{j}(y)$. From this, we see

$$
f_{k}(x) \geq f_{k}(y)-d(x, y)>p(y)-d(x, y)-R-\delta .
$$

Case 1: $p(y) \geq d(x, y)$. Then we have $p(x)=p(y)-d(x, y)$, and

$$
f_{k}(x)>p(x)-R-\delta .
$$

Case 2: $p(y) \leq d(x, y)$. Then $p(x)=0$ and

$$
f_{k}(x) \geq 0>p(x)-R-\delta
$$

holds trivially.

On the other hand, we have

$$
f_{k}(x) \leq \bigvee_{j \in J} f_{j}(x) \leq p(x)+R<p(x)+R+\delta \quad \forall x \in Y
$$

and thus $d_{\infty}\left(f_{k}, p\right)<R+\delta$. The proof is illustrated by Figure 2.4.

(2) $\Rightarrow(\mathbf{1})$ : The family of all bounded $\Lambda$-functions is a metrically closed base according to Corollary 97, and due to Proposition 78 the bounded completely ml-irreducible elements form a subset in them.

The formula

$$
d_{\infty}\left(p, \bigvee_{j \in J} f_{j}\right) \leq R \Rightarrow \forall \delta>0 \exists j \in J: d_{\infty}\left(p, f_{j}\right) \leq R+\delta
$$

of Definition 173 does not hold for $\delta=0$, as one can see in Lemma 102 As a counter-example, insert $\Lambda(y, 1)=\bigvee_{r \in(0,1)} \Lambda(y, r)$. This is exactly the difference between a completely irreducible and a completely ml-irreducible element.

Recalling the short note after Corollary 95, the metric information of $Y$ is encoded in the $\Lambda$-functions and the distances between them. However, these 


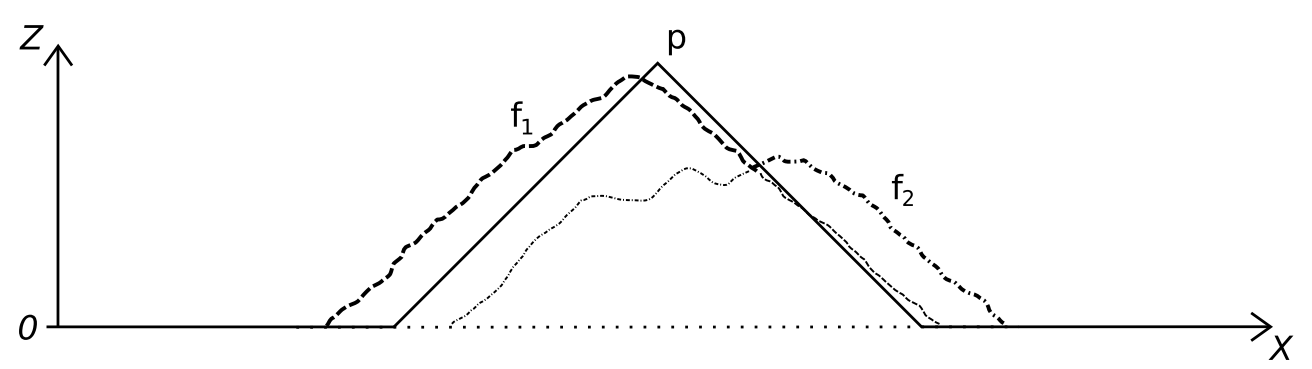

Figure 2.4: When approximating a $\Lambda$-function $p$ by Lipschitz functions $f_{j}$, one of the functions (here $f_{1}$ ) must approximate the maximum point of $p$. This function may not decrease too fast (Lipschitz!), and may not increase too fast, as it is bounded from above by the approximation of $p$, hence it already approximates $p$ on its own, see Lemma 102.

functions are at first sight just some arbitrary subset of $\operatorname{Lip} Y$ and thus there seems to be no hope for the metric $c_{+}$space $\left(\operatorname{Lip} Y, d_{\infty}\right)$ to hold the full information about $Y$ 's metric. The preceding Lemma now explains to us that the (bounded) $\Lambda$-functions are not arbitrary at all - they have a specific, lattice theoretic property that distinguishes them from the remaining functions. Hence, in some sense the metric information of $Y$ is now part of the combined metric and lattice structure of $\operatorname{Lip} Y$.

Corollary 103

Let $X, Y$ be complete, $\epsilon \geq 0$ and $\kappa: \operatorname{Lip} Y \rightarrow \operatorname{Lip} X$ an $\epsilon$-ml-isomorphism. Then $\kappa$ maps bounded $\Lambda$-functions $6 \epsilon$-near bounded $\Lambda$-functions.

Proof Follows from Lemmas 92 and 102 .

The preceding Corollary is the critical point in our analysis: We can use $\Lambda$ functions as building blocks for Lipschitz functions, as Proposition 96 tells us. From Corollary 103 we now know that these building blocks (or, at least, the bounded versions) behave sensible under $\epsilon$-ml-isomorphisms $\kappa$, such that we only have to understand how they are mapped by $\kappa$ to reconstruct all other Lipschitz functions. In particular, as they are strongly connected to the underlying spaces, they allow us to define mappings between them, as will be shown next.

\subsection{Inducing Rough Isometries}

In this section, we show the reversal of Theorem 101 Given an $\epsilon$-ml-isomorphism $\kappa$ we construct a rough isometry $\eta$ such that $\bar{\eta}$ is near $\kappa$. 
Lemma 104

Let $X, Y$ be complete, and $\epsilon \geq 0$ arbitrary. Let $\kappa: \operatorname{Lip} Y \rightarrow \operatorname{Lip} X$ be an $\epsilon$-ml-isomorphism, $\epsilon \geq 0$. Then there is a map $\eta: X \rightarrow Y$ such that

$$
d_{\infty}\left(\Lambda(\eta x, r), \kappa^{\prime}(\Lambda(x, r))\right) \leq 59 \epsilon
$$

for all $x \in X, r \in[0, \infty]$. For $r \in[38 \epsilon, \infty)$, we may replace " $59 \epsilon$ " by " $43 \epsilon "$ ".

Proof In the following proof, the first two cases will deal with $\epsilon>0$ and finite $r$, the third with $\epsilon=0$ and finite $r$ and the fourth with $r=\infty$.

Case 1 and 2: For each $x \in X$, choose $\eta(x) \in Y$ and $s_{x} \in[0, \infty)$ such that $\Lambda\left(\eta x, s_{x}\right)$ is $6 \epsilon$-near $\kappa^{\prime} \Lambda(x, 22 \epsilon)$ (use Corollary 103).

Case 1: $\epsilon>0, r \in[38 \epsilon, \infty)$. Let $\Lambda\left(x^{\prime}, r^{\prime}\right)$ be $6 \epsilon$-near $\kappa^{\prime} \Lambda(x, r)$. Then by Proposition 90 holds

$$
\begin{aligned}
d_{\infty}(0, \Lambda(x, r))=r \Rightarrow\left|d_{\infty}\left(0, \kappa^{\prime} \Lambda(x, r)\right)-r\right| & \leq 2 \epsilon \\
\Rightarrow \quad\left|r^{\prime}-r\right| & \leq 8 \epsilon .
\end{aligned}
$$

In the same way, we have

$$
\begin{aligned}
\left|d_{\infty}\left(0, \Lambda\left(\eta x, s_{x}\right)\right)-d_{\infty}\left(0, \kappa^{\prime} \Lambda(x, 22 \epsilon)\right)\right| & \leq 6 \epsilon \\
\Rightarrow \quad\left|s_{x}-22 \epsilon\right| & \leq 8 \epsilon .
\end{aligned}
$$

We now take a look at

$$
\begin{aligned}
d_{\infty}(\Lambda(x, r), \Lambda(x, 22 \epsilon)) & =r-22 \epsilon \quad(\text { as } r \geq 22 \epsilon) \\
\Rightarrow \quad\left|d_{\infty}\left(\kappa^{\prime} \Lambda(x, r), \kappa^{\prime} \Lambda(x, 22 \epsilon)\right)-(r-22 \epsilon)\right| & \leq \epsilon \\
\Rightarrow \quad\left|d_{\infty}\left(\Lambda\left(x^{\prime}, r^{\prime}\right), \Lambda\left(\eta x, s_{x}\right)\right)-(r-22 \epsilon)\right| & \leq 13 \epsilon .
\end{aligned}
$$

Now we calculate $d:=d_{\infty}\left(\Lambda\left(x^{\prime}, r^{\prime}\right), \Lambda\left(\eta x, s_{x}\right)\right)$ by hand. From Proposition 94 , $d$ could be $r^{\prime} \vee s_{x}$ or $d\left(x^{\prime}, \eta x\right)+\left|r^{\prime}-s_{x}\right|$. We know

$$
s_{x} \leq 8 \epsilon+22 \epsilon=30 \epsilon \leq r-8 \epsilon \leq r^{\prime},
$$

hence $r^{\prime} \vee s_{x}=r^{\prime}$. However, as $d \leq r-22 \epsilon+13 \epsilon=r-9 \epsilon$, but $r^{\prime} \geq r-8 \epsilon, d$ cannot be $r^{\prime}$ (here we use $\epsilon>0$ ). Remains

$$
d=d\left(x^{\prime}, \eta x\right)+\left|r^{\prime}-s_{x}\right| \quad \text { with } \quad|d-(r-22 \epsilon)| \leq 13 \epsilon .
$$

As shown above, $r^{\prime} \geq s_{x}$, hence

$$
\begin{aligned}
d\left(x^{\prime}, \eta x\right) & \leq r-22 \epsilon+13 \epsilon-\left|r^{\prime}-s_{x}\right|=r-22 \epsilon+13 \epsilon-r^{\prime}+s_{x} \\
& \leq r-22 \epsilon+13 \epsilon-r+8 \epsilon+22 \epsilon+8 \epsilon=29 \epsilon .
\end{aligned}
$$

This, and $\left|r^{\prime}-r\right| \leq 8 \epsilon$, yield

$$
\begin{aligned}
d_{\infty}\left(\Lambda(\eta x, r), \Lambda\left(x^{\prime}, r^{\prime}\right)\right) & \leq d\left(x^{\prime}, \eta x\right)+\left|r-r^{\prime}\right| \leq 37 \epsilon \\
\Rightarrow \quad d_{\infty}\left(\Lambda(\eta x, r), \kappa^{\prime} \Lambda(x, r)\right) & \leq 43 \epsilon .
\end{aligned}
$$




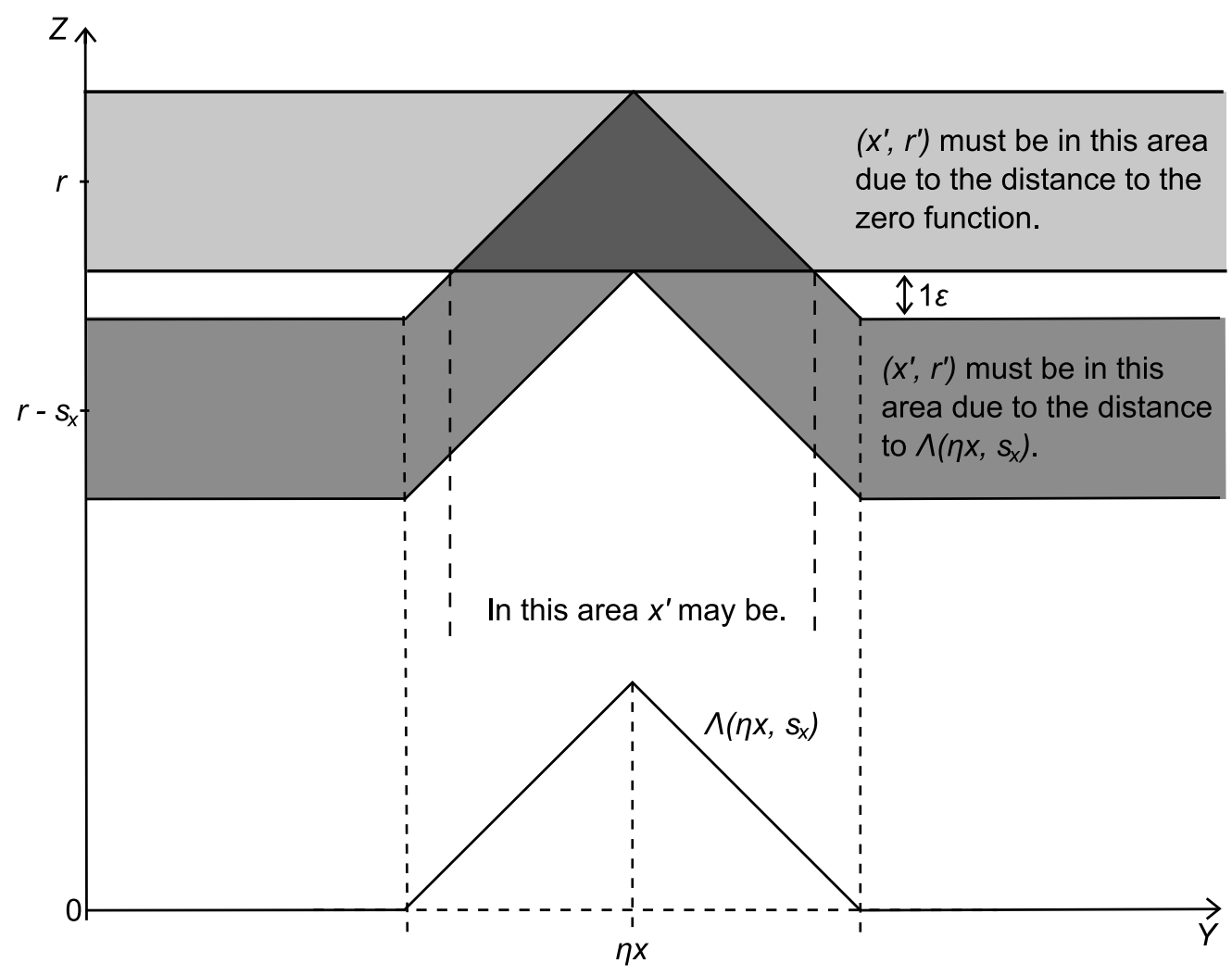

Figure 2.5: The function $\Lambda\left(x^{\prime}, r^{\prime}\right)$ in the proof of Lemma 104 is already determined up to nearness by its distance to two other functions: the zero function and $\Lambda\left(\eta x, s_{x}\right)$. This shows: A $\Lambda$-function $\Lambda(y, s)$ is not only mapped near another $\Lambda$-function $\Lambda\left(y^{\prime}, s^{\prime}\right)$, but $y^{\prime}$ only depends on $y$ and $s^{\prime}$ only depends on $s$ (modulo some multiples of $\epsilon$ ).

Case 2: $\epsilon>0, r \in[0,38 \epsilon)$. Obviously,

$$
\begin{aligned}
d_{\infty}\left(\Lambda(\eta x, r), \kappa^{\prime} \Lambda(x, r)\right) \leq \quad & d_{\infty}\left(\Lambda(\eta x, r), \Lambda\left(\eta x, s_{x}\right)\right) \\
+ & d_{\infty}\left(\Lambda\left(\eta x, s_{x}\right), \kappa^{\prime} \Lambda(x, 22 \epsilon)\right) \\
+ & d_{\infty}\left(\kappa^{\prime} \Lambda(x, 22 \epsilon), \kappa^{\prime} \Lambda(x, r)\right) \\
\leq \quad & \left|r-s_{x}\right|+6 \epsilon+\epsilon+|r-22 \epsilon|
\end{aligned}
$$

As $r \in[0,38 \epsilon)$ and $s_{x} \in[14 \epsilon, 30 \epsilon]$ (see above), we receive $\left|r-s_{x}\right| \leq 30 \epsilon$ and $|r-22 \epsilon| \leq 22 \epsilon$. This adds up to $59 \epsilon$.

Case 3: $\epsilon=0, r \in[0, \infty)$. As of Corollary 103, for all $x \in X$ we can choose $\eta(x)$ such that $\kappa^{\prime} \Lambda(x, 1)=\Lambda\left(\eta x, s_{x}\right)$ for some $s_{x} \in[0, \infty)$. From Proposition 90 we see $\kappa^{\prime}(0)=0$, hence $s_{x}=1$. Now, let $r \in[0, \infty)$ be arbitrary. Let $x^{\prime} \in Y$, $r^{\prime} \in[0, \infty]$ such that $\kappa^{\prime} \Lambda(x, r)=\Lambda\left(x^{\prime}, r^{\prime}\right)$. Clearly, from the distance to 0 we again have $r^{\prime}=r$. From

$$
d_{\infty}(\Lambda(x, 1), \Lambda(x, r))=|r-1|
$$


we conclude

$$
d_{\infty}\left(\Lambda(\eta x, 1), \Lambda\left(x^{\prime}, r\right)\right)=|r-1| .
$$

Due to Proposition 94 this can happen iff (a) $|r-1|=1 \geq r$ or (b) $|r-1|=r \geq 1$ or (c) $d\left(x^{\prime}, \eta x\right)=0$. Case (c) proves our statement, case (b) cannot happen: $|r-1|=r$ iff $r=\frac{1}{2}$, which contradicts $r \geq 1$. So, assume case (a). Then $r=2$, which contradicts $r \leq 1$, or $r=0$. But the case $r=0$ is trivial, as we already saw from Proposition 90 that

$$
\kappa^{\prime} \Lambda(x, 0)=0=\Lambda(\eta x, 0) .
$$

Case 4: $r=\infty$. We know $\Lambda(x, \infty)=\bigvee_{s \in[38 \epsilon, \infty)} \Lambda(x, s)$. Using our result for finite $r$, we conclude

$$
d_{\infty}\left(\kappa^{\prime} \bigvee_{s \in[38 \epsilon, \infty)} \Lambda(x, s), \bigvee_{s \in[38 \epsilon, \infty)} \Lambda(\eta x, s)\right) \leq 1 \epsilon+43 \epsilon
$$

Apply $\bigvee_{s \in[38 \epsilon, \infty)} \Lambda(\eta x, s)=\Lambda(\eta x, \infty)$ to see that $\kappa^{\prime} \Lambda(x, \infty)$ is indeed $44 \epsilon$-near $\Lambda(\eta x, \infty)$.

Lemma 105

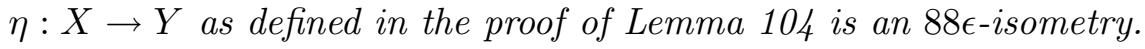

Proof From Corollary 95 follows

$$
d(\eta x, \eta y)=\lim _{r \rightarrow \infty, r \neq \infty} d_{\infty}(\Lambda(\eta x, r), \Lambda(\eta y, r)) .
$$

Applying Lemma 104 for large enough $r$ yields:

$$
\left|d_{\infty}(\Lambda(\eta x, r), \Lambda(\eta y, r))-d_{\infty}\left(\kappa^{\prime} \Lambda(x, r), \kappa^{\prime} \Lambda(y, r)\right)\right| \leq 2 \cdot 43 \epsilon
$$

and of course

$$
\left|d_{\infty}\left(\kappa^{\prime} \Lambda(x, r), \kappa^{\prime} \Lambda(y, r)\right)-d_{\infty}(\Lambda(x, r), \Lambda(y, r))\right| \leq \epsilon .
$$

Hence

$$
|d(\eta x, \eta y)-d(x, y)| \leq 87 \epsilon,
$$

i.e. $\eta$ is a rough isometric embedding. Just as $\eta$ was constructed from $\kappa^{\prime}$, we construct $\eta^{\prime}$ from $\kappa$. It remains to show that $\eta \circ \eta^{\prime}$ and $\eta^{\prime} \circ \eta$ are near identities. Again, we make use of Corollary 95

$$
\Rightarrow \begin{aligned}
\left|d\left(\eta \eta^{\prime} x, x\right)-\lim _{r \rightarrow \infty, r \neq \infty} d_{\infty}\left(\Lambda\left(\eta \eta^{\prime} x, r\right), \Lambda(x, r)\right)\right| & =0 \\
\left|d\left(\eta \eta^{\prime} x, x\right)-\lim d_{\infty}\left(\kappa^{\prime} \kappa \Lambda(x, r), \Lambda(x, r)\right)\right| & \leq 2 \cdot 43 \epsilon+\epsilon \\
\Rightarrow d\left(\eta \eta^{\prime} x, x\right) & \leq 88 \epsilon
\end{aligned}
$$

Same for $\eta^{\prime} \circ \eta$. 
Theorem 106 (= Th. 3) 106

Let $X, Y$ be complete metric + spaces and $\epsilon \geq 0$. For each $\epsilon$-ml-isomorphism $\kappa: \operatorname{Lip}(Y) \rightarrow \operatorname{Lip}(X)$ there is an $88 \epsilon$-isometry $\eta: X \rightarrow Y$, such that $\kappa$ is



Proof Construct $\eta$ as in Lemma 104. It is an 88 6 -isometry due to Lemma 105. It remains to show that $\kappa$ is near $\bar{\eta}$ : Let $f \in \operatorname{Lip} Y$ be arbitrary. Represent $f$ via $\Lambda$-functions as in Proposition 96. Obviously,

$$
d_{\infty}\left(\kappa \bigvee_{y \in Y} \Lambda(y, f y), \bigvee_{y \in Y} \Lambda\left(\eta^{\prime} y, f y\right)\right) \leq 1 \epsilon+59 \epsilon
$$

due to Lemma 104. Apply Proposition 98

\subsection{Other Metrics for $\operatorname{Lip} X$}

We have seen in Chapter 1 that a lattice like Lip $X$ can be equipped with a large variety of different metrics. We want to take a look at some of them.

Example 107 (Discrete Metric or Topology) 107

The discrete metric is an ultravaluation of Example 59 for a positive and constant function $\kappa: X \rightarrow[0, \infty]$. We have further seen that complete irreducibility and complete ml-irreducibility are the same in this metric in Section 1.4. More generally, when the topology derived from the metric is discrete, complete ml-irreducibility implies complete irreducibility. However, as long as $X$ is nonempty, the only completely irreducible element in $\operatorname{Lip} X$ is the zero function.

This is reflected in the inability to state a Smoothening Theorem for (Lip $\left.X, d_{\mathrm{dis}}\right)$ in the way of Theorem 82: $\operatorname{Lip} X$ and $\operatorname{Lip} Y$ are trivially always $\epsilon$-ml-isometric for $\epsilon>\operatorname{diam} \operatorname{Lip} X \vee \operatorname{diam} \operatorname{Lip} Y$; otherwise, they must be perfectly lattice isomorphic. For the most basic example, $\# X=1$ and $\# Y=2$, this already fails.

Example $108\left(L^{1}\right.$-Metric $) \quad \ldots \ldots \ldots \ldots \ldots \ldots \ldots \ldots \ldots \ldots \ldots \ldots \ldots . \ldots \ldots$ The most simple valuation metric on $\operatorname{Lip} X$ for a measure space $(X, \mu)$ is the $L^{1}$-metric from Example 56, and a very canonical choice for many applications in $\operatorname{Lip} X$; however, it is not a good choice in handling with coarse geometry. Let $X=Y=\mathbb{R}^{2}$ be the Euclidean plane, $\epsilon>0$ arbitrary, and

$$
\begin{aligned}
\eta: \quad X & \rightarrow Y \\
(r, \phi) & \mapsto(0 \vee(r-\epsilon), \phi)
\end{aligned}
$$

in polar coordinates. $\eta$ is an "additive contraction" of the Euclidean plane, where each point moves a distance $\epsilon$ nearer to the origin, while all elements inside the $\epsilon$-ball around the origin are directly mapped to it. $\eta$ obviously is 
a $2 \epsilon$-isometry. Let $\mu$ be the standard Lebesgue measure on $X$ and $Y$. Let $f=\Lambda((0,0), r)$ for $r \in[0, \infty]$, then $d_{1}(0, f)$ is exactly the volume of a cone of height $r$ and radius $r$, i.e. $r^{3} \cdot \pi / 3$. On the other hand, $f \circ \eta$ already is Lipschitz (hence it equals $\overline{f \circ \eta}$ ), and is the frustrum of a cone of height $r$, radius $r+\epsilon$, and projected height $r+\epsilon$, hence its distance to zero is:

$$
d_{1}(0, f \circ \eta)=\frac{\pi}{3} \cdot\left((r+\epsilon)^{3}-\epsilon^{3}\right)
$$

which is far, far away from $d_{1}(0, f)$. This problem cannot be avoided even when switching the codomain to an interval of $\mathbb{R}$, and each other $L^{p}$-metric for $1<p<\infty$ suffers from it.

In a similar way one shows that no positive $\Lambda$-function is completely ml-irreducible: Let $p=\Lambda((0,0) r)$ with $r>\epsilon$ and $f_{j}=\Lambda(j, r-\epsilon)$ with $j \in J=$ $B_{(0,0)}(\epsilon)$. Then $d\left(p, \bigvee f_{j}\right)$ is $\epsilon^{3} \cdot \pi / 3$, but $d\left(p, f_{j}\right)=\left(r^{3}-(r-\epsilon)^{3}\right) \cdot \pi / 3$, hence of order $r^{2}$. On the other hand, we know from Proposition 78 and Corollary 97 that all completely ml-irreducible elements must be in the family of bounded $\Lambda$-functions (this property is independent of the metric). Hence, in this special case, there is no completely ml-irreducible element other than the zero function.

Example 109 (Lipschitz Norm) ....................... 109

Let us consider Weaver's Lipschitz norm from Example [69. In addition to the supremum metric, it additionally measures the Lipschitz constant of the difference function. In $\operatorname{Lip} X$, this is bounded by 1 , and should not provide a problem for $\epsilon>1$. Hence, choose $\epsilon<1$, furthermore $\# X=1, Y=\{a, b\}$ with diameter $\epsilon$, and $\eta: X \rightarrow Y$ simply $\mathrm{pt} \mapsto$ a. Take $f: Y \rightarrow[0, \infty]$ to be $(0, \epsilon)$. Then the Lipschitz norm of $f$ clearly is 1 , but $\|f \circ \eta\|_{L}=0$. Thus there cannot be an analog of Theorem 82 for the Lipschitz norm for $\epsilon<1$ (or $<K$, respectively).

A similar theme would be to explore the rough isometries of Hajtasz-Sobolev spaces ([He], chapter 5). These are subsets of $L^{p}$ function spaces, with a norm similar to the Sobolev norm and hence similar to Weaver's Lipschitz norm. Similar counter-examples as the one above should hold for Hajtasz-Sobolev spaces.

Example 110 (Peak Metric)

We take a look at the peak metric defined in 61. This ultrametric is very similar to the discrete metric, in that no non-trivial $\Lambda$-function is completely mlirreducible (take $f_{j}=\Lambda\left(x, r_{j}\right)$ with $r_{j} \nearrow r$ to approach $p=\Lambda(x, r)$, with $\left.d\left(p, f_{j}\right)=r\right)$. As counter-example we make use of the spaces in Example 109, but with $f=(r, r+\epsilon)$ and $g=(r, r)$ for some large enough $r \in[0, \infty)$. Then $d(f, g)=r+\epsilon$, but $d(f \circ \eta, g \circ \eta)=0$.

Example 111 (Basepoint Metrics)

We finish with the inner and outer basepoint metrics we defined in 61. based at $x_{0} \in X$. The outer basepoint metric shares certain similarities with the discrete metrics, but there are lots of accumulation points in $\operatorname{Lip} X$ (every function $f$ which takes value 0 at least once), and the metric is unbounded. Choose 
$R \in(0, \infty)$ large enough, take $X=[0, R] \subseteq \mathbb{R}, Y=[0, R+\epsilon]$, $\eta$ the canonical embedding (which is an $\epsilon$-isometry). Choose $f=\Lambda(R+\epsilon, \epsilon)$. Then $d(f, 0)=R+\epsilon$, but $d(f \circ \eta, 0 \circ \eta)=0$.

For the inner basepoint metric, the inverse of the above counter-example applies: Consider $Y=[0, \epsilon]$ with basepoint 0 , and $X=\{0\}$, with standard embedding $\eta$. Take $f=\Lambda(\epsilon, \epsilon)$. Then $d(f, 0)=1$ (as $f$ and 0 differ at each point $x>0)$, but $d(f \circ \eta, 0 \circ \eta)=0$.

In both cases there again are no non-trivial completely ml-irreducible elements.

In view of these Examples, we now feel confident to state the following conjecture:

Conjecture 112

The statement " $X$ is $\epsilon$-isometric to $Y$, then $\left(\operatorname{Lip} X, d_{X}\right)$ and $\left(\operatorname{Lip} Y, d_{Y}\right)$ are $6 \epsilon$-ml-isomorphic" is true if and only if each $\Lambda$-function in $\operatorname{Lip} X$ and $\operatorname{Lip} Y$ is completely ml-irreducible relative to $d_{X}$ and $d_{Y}$, respectively. (There might be trivial exceptions.)

The use of other types of functions mostly is of similar failure:

Example 113

Take $X=\{0\} \subseteq Y=\{0,1\} \subseteq \mathbb{R}$ and $\eta$ the inclusion, $\epsilon=1$. The metric $c_{+}$spaces of $[0, \infty]$-valued continuous functions $C(X)$ and $C(Y)$ with supnorm are isomorphic to $[0, \infty]$ and $[0, \infty]^{2}$ respectively, which are not roughly isometric.

\subsection{Quasi-Isometries}

An obvious generalization is the question, whether similar statements as those of Theorems 101 and 106 hold for quasi-isometries instead of rough isometries. Although many ideas still work in the context of quasi-isometries, a function's Lipschitz constant is distorted in the process of Lemma 99 Hence there happens

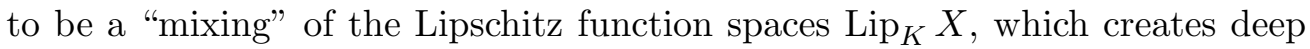
problems. Indeed, it is always possible to split a quasi-isometry $\eta: X \rightarrow Y$ into two rough isometries $\eta_{X}: X \rightarrow X_{0}$ and $\eta_{Y}: Y_{0} \rightarrow Y$ and a bilipschitz mapping $\eta^{\prime}: X_{0} \rightarrow Y_{0}$ by introducing sufficiently $\epsilon$-dense and $\epsilon$-discrete nets $X_{0} \subseteq X$ and $Y_{0} \subseteq Y$, so the problem completely reduces to the problem for bilipschitz mappings.

Example 114

Let $X=\mathbb{R}$, and $\eta: X \rightarrow X$ be the function which is the identity on the negatives and doubles each non-negative argument. $\eta$ is bilipschitz with bilipschitzconstant 2. Let $f$ be any 1-Lipschitz function, then $f \circ \eta$ can be anything between 1-Lipschitz and 2-Lipschitz. To allow for further comparisons between different 
functions, one has to change the function spaces: Just switching to $\operatorname{Lip}_{2} X$ in this case will not suffice, as $\eta\left(\operatorname{Lip}_{1} X\right)$ is not roughly dense in $\operatorname{Lip}_{2} X$. And combining all of them into $\bigcup_{K \geq 0} \operatorname{Lip}_{K} X$ is not compatible with the Lipschitzization of Lemma 99, as the necessary $\epsilon$ cannot be bounded. However, there are chances to define generalized Lipschitz functions of the kind

$$
d(f x, f y) \leq c(x, y) \cdot d(x, y)
$$

where $c: X \times X \rightarrow\left(c_{0}^{-1}, c_{0}\right)$ is a fixed function, bounded by $c_{0} \in[1, \infty)$. c may absorb the distortion by the quasi-isometry, but this approach uglily depends on the quasi-isometry itself and is of inferior expressiveness.

\subsection{Scaling limits}

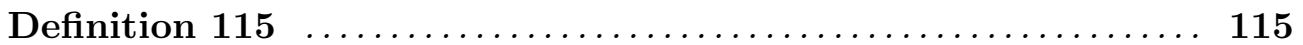

Let the rough distance $d_{R}(X, Y)$ between two metric $c_{+}$spaces $X$ and $Y$ be the infimum over all $\epsilon \geq 0$ such that $X$ and $Y$ are $\epsilon$-isometric, or $\infty$ if there are none. If $d_{R}(X, Y)=0$, the spaces $X$ and $X^{\prime}$ will be called a pseudo-isometric.

The rough distance fulfills a triangle-inequality, as concatenation of an $\epsilon$ - and a $\delta$-isometry is an $(\epsilon+\delta)$-isometry. It is closely related to the Gromov-HausdorffDistance for compact spaces, but may differ in a variable between $\frac{1}{2}$ and 2 (i.e. they are Lipschitz-equivalent, see e.g. Gv2, Proposition 3.5).

Pseudo-isometry is a little bit less than isometry. However, they are equivalent if only compact spaces are compared (e.g. [P], Gv2]), or if we deal with simple graphs, due to their integer metric. A nice article about scaling limits, Gromov-Hausdorff distances and quasi-isometries in the case of graphs and Cayley graphs is $\mathbf{R e}$.

\section{Definition 116}

Let Met* be the (non-small) groupoid of all pseudo-isometry-classes of metric $c_{+}$spaces with $\epsilon$-isometries as morphisms. $d_{R}$ is a metric $c_{+}$on Met* $^{*}$ in a natural way (apart from the fact that $\mathbf{M e t}^{*}$ is no set).

Each of the components of Met* can be endowed with a metric and topology, with the only drawback of being proper classes. This "topology" allows us to define the convergence of metric + spaces to another metric + space, up to pseudo-isometry. Met* is complete in this "topology" (cf. [P], Proposition 6, the proof works in non-compact and non-separable cases as well).

Definition 117

For any $\ell>0$ define $s_{\ell}:$ Met $^{*} \rightarrow$ Met $^{*}$ by

$$
s_{\ell}[(X, d)]:=[(X, \ell \cdot d)],
$$




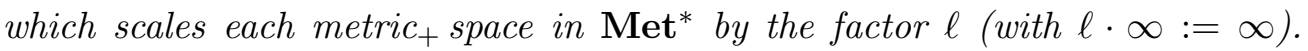
This operation clearly is compatible with pseudo-isometry. Let $[X]$ be a class of spaces in Met*. If the limit $^{*}$

$$
s[X]:=\lim _{\ell \rightarrow 0} s_{\ell}[X]
$$

exists for all sequences $\ell \rightarrow 0$, then $s[X]$ (resp. all members of $s[X]$ ) is called the (strong) scaling limit of $[X]$.

We now want to apply Theorem 101

Corollary 118

Let $X, Y$ be some metric spaces, such that $Y$ is a strong scaling limit of $X(Y$ is unique up to pseudo-isometry). Then there is a strong scaling limit of $\operatorname{Lip} X$, and it is pseudo-isometric to $\operatorname{Lip} Y$. ("The scaling limit of the Lipschitz space is the Lipschitz space of the scaling limit, modulo pseudo-isometry.")

Proof $\operatorname{As} d_{R}\left(Y, s_{\ell} X\right) \rightarrow 0$ for $\ell \rightarrow 0$, there are $\epsilon_{\ell}$-isometries $\eta_{\ell}: s_{\ell} X \rightarrow Y$ with $\epsilon_{\ell} \rightarrow 0$. These induce $4 \epsilon_{\ell}$-ml-isomorphisms $\bar{\eta}_{\ell}: \operatorname{Lip} Y \rightarrow \operatorname{Lip} s_{\ell} X$, which are in particular $4 \epsilon_{\ell}$-isometries. Hence, $d_{R}\left(\operatorname{Lip} Y\right.$, Lip $\left.s_{\ell} X\right) \rightarrow 0$. Proper rescaling of the associated Lipschitz functions further shows $s_{\ell} \operatorname{Lip} X$ is naturally isometric to $\operatorname{Lip} s_{\ell} X$, hence $s_{\ell} \operatorname{Lip} X \rightarrow \operatorname{Lip} Y$ up to pseudo-isometry.

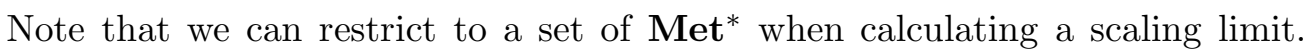
Thus, we can make use of Banach's Fixed Point Theorem.

We may now define the groupoid Lip Met* with objects Lip $X$ for each metric space $X$, with distance function

$$
d_{\mathrm{ml}}(\operatorname{Lip} X, \operatorname{Lip} Y):=\inf \{\epsilon \geq 0: \exists \kappa: \operatorname{Lip} Y \rightarrow \operatorname{Lip} X \epsilon \text {-ml-isom. }\}
$$

modulo pseudo-ml-isometry $d_{\mathrm{ml}}=0$. We endow Lip Met* with rough mlisomorphisms as morphisms. In these terms, the mapping ${ }^{-}: \eta \mapsto \bar{\eta}$ is a Lipschitz equivalence between the metric categories Met* and Lip Met*, and a contravariant functor up to nearness of rough isometries.

Unfortunately, we are not yet able to generalize Theorem 101 to $\epsilon$-short maps and $\epsilon$-ml-short maps, which would be the appropriate morphisms of Met and Lip Met (see Section 0.1.2). 


\section{Chapter 3}

\section{Rough Isometries of Groups}

\subsection{The Theorem of Mazur-Ulam}

Some Theorems and Lemmas have the property of being stable against perturbations of their input. We want to give an example for this in form of a variant of the Banach Fixed Point Theorem. For this, we copy the standard proof from [Ho] and replace all steps in the proof by their rough counterparts: A point is replaced by a ball, uniqueness is replaced by bounded distance, and so on.

Theorem 119

Let $M$ be a non-empty true metric space (not necessarily complete), and $T$ : $M \rightarrow M$ such that there are $0 \leq q<1$ and $\epsilon \geq 0$ with

$$
d(T x, T y) \leq q d(x, y)+\epsilon
$$

for all $x, y \in M$. Then for each $R>\epsilon(2-q) /(1-q)^{2}$ there is a point $x_{0} \in M$ such that $T B_{R}\left(x_{0}\right) \subseteq B_{R}\left(x_{0}\right)$, and any two such points are within distance $\leq(2 R+\epsilon) /(1-q)$.

Proof Let $r:=R(1-q)-\epsilon>\epsilon /(1-q)$. By iteration we see that

$$
\begin{aligned}
d\left(T^{n} x, T^{n} y\right) & \leq q^{n} d(x, y)+\left(1+q+q^{2}+\ldots+q^{n-1}\right) \cdot \epsilon \\
& \leq q^{n} d(x, y)+\epsilon /(1-q)
\end{aligned}
$$

holds for all $n \in \mathbb{N}_{0}$. Let $x \in M$ be arbitrary, then follows

$$
d\left(T^{n} x, T^{n-1} x\right) \leq q^{n-1} d(T x, x)+\epsilon /(1-q) .
$$

As $q^{n} \rightarrow 0$ for $n \rightarrow \infty$, there is $N \in \mathbb{N}_{*}$ and $x_{0}:=T^{N} x$ with

$$
d\left(T x_{0}, x_{0}\right) \leq r
$$

(This is the rough counterpart of the Cauchy criterion.) For all $y \in B_{R}\left(x_{0}\right)$

$$
d\left(T y, x_{0}\right) \leq d\left(T y, T x_{0}\right)+d\left(T x_{0}, x_{0}\right) \leq q R+r+\epsilon=R
$$


holds, hence $T B_{R}\left(x_{0}\right) \subseteq B_{R}\left(x_{0}\right)$. Now let $y_{0} \in M$ be another point with $T B_{R}\left(y_{0}\right) \subseteq B_{R}\left(y_{0}\right)$. Then

$$
\begin{aligned}
d\left(x_{0}, y_{0}\right) & \leq d\left(x_{0}, T x_{0}\right)+d\left(T x_{0}, T y_{0}\right)+d\left(T y_{0}, y_{0}\right) \\
& \leq 2 R+q d\left(x_{0}, y_{0}\right)+\epsilon \\
\Rightarrow d\left(x_{0}, y_{0}\right) & \leq(2 R+\epsilon) /(1-q) .
\end{aligned}
$$

This procedure works for all proofs of sufficient simplicity, which are straightforward applications of (in)equalities or quantifiers. For example, each of the intervaluation laws derivable by a finite Venn diagram (see 64) still roughly holds when the cut law of Definition 62 is replaced by

$w(f, g \vee h) \circ_{w} w(f \wedge h, g)-\epsilon \leq w(f, g) \leq w(f, g \vee h)+w(f \wedge h, g)+\epsilon$.

Unfortunately, not every proof can be reformulated in a rough context. Here, we will first quote a very elegant proof of the Mazur-Ulam Theorem (Mㅣ] ), as given by Väisälä in $[\mathbf{V}]$. We then conjecture a rough version of the Mazur-Ulam Theorem, and show how Väisälä's proof fails to adapt to the rough context. (Note in comparison that with an "isometry" we always mean a bijective isometry.)

Theorem 120 (Mazur-Ulam)

Every isometry $f: E \rightarrow F$ between normed finite-dimensional vector spaces is affine (i.e. linear plus constant).

Proof We quote from $[\underline{V}]$ : Let $a, b \in E$ be arbitrary, and put $z:=(a+b) / 2$. Let $W$ be the family of all isometries with fixed points $a$ and $b$. Let $\lambda:=$ $\sup \{\|g(z)-z\|: g \in W\}$. As $a$ is a fixed point of each $g \in W$, we have $\|g(z)-a\|=\|g(z)-g(a)\|=\|z-a\|$, and

$$
\|g(z)-z\| \leq\|g(z)-a\|+\|a-z\|=2 \cdot\|a-z\|,
$$

hence $\lambda$ is finite.

Let $\psi$ be the reflection of $E$ in $z$, this is $\psi: x \mapsto 2 z-x$. For each $g \in W$ holds $g^{*}=\psi g^{-1} \psi g \in W$ as well, as $\psi$ maps $a$ and $b$ onto each other. $\psi$ is an isometry, and its only fixed point is $z$. This implies

$$
\text { and } \begin{aligned}
\|\psi(x)-z\| & =\|x-z\| \\
\text { at } \quad\|\psi(x)-x\| & =2\|x-z\| .
\end{aligned}
$$

Now, $g$ and $g^{-1}$ are isometries, so we find

$$
\begin{aligned}
2\|g(z)-z\| & =\|\psi(g(z))-g(z)\|=\left\|\left(g^{-1} \psi g\right)(z)-z\right\| \\
& =\left\|\left(\psi g^{-1} \psi g\right)(z)-z\right\|=\left\|g^{*}(z)-z\right\| \leq \lambda .
\end{aligned}
$$


For each $\delta>0$ we may choose $g \in W$ with $\|g(z)-z\| \geq \lambda-\delta$, which yields $2 \lambda \leq \lambda+\delta$, consider $\delta \rightarrow 0$ and find $\lambda=0$, thus $g(z)=z$ whenever $g$ is an isometry with fixed points $a$ and $b$.

Now let $f: E \rightarrow F$ be any isometry, and let $z^{\prime}:=(f(a)+f(b)) / 2$. Let $\psi^{\prime}$ be the reflection at $z^{\prime}$, then $h:=\psi f^{-1} \psi^{\prime} f$ fixes $a$ and $b$ ( $a$ is first mapped to $f(a)$, then to $f(b)$, to $b$, and finally back to $a$ by $\psi)$, hence $h(z)=z$. But this means $\left(\psi f^{-1} \psi^{\prime} f\right)(z)=z$, and, as $\psi^{-1}(z)=z$, simply $\psi^{\prime}(f(z))=f(z)$. As $\psi^{\prime}$ is a reflection, there is only one fixed point, and this is $z^{\prime}$; hence, we have $z^{\prime}=f(z)$, and thereby

$$
f\left(\frac{a+b}{2}\right)=\frac{f(a)+f(b)}{2} .
$$

Now define $g(x):=f(x)-f(0)$. From direct calculation follows

$$
g\left(\frac{x+y}{2}\right)=\frac{g(x)+g(y)}{2} .
$$

for all $x, y \in E$. Insert $y=0$ to find $g(2 x)=2 g(x)$, and subsequently

$$
g(x+y)=\frac{1}{2} g(2 x)+\frac{1}{2} g(2 y)=g(x)+g(y) .
$$

Furthermore, we have

$$
g\left(\sum_{j \in J} 2^{j} \cdot x\right)=\sum_{j \in J} 2^{j} \cdot g(x)
$$

for any finite $J \subseteq \mathbb{Z}$. Continuity of $g$ yields its full linearity.

Conjecture 121 (Mazur-Ulam, rough version)

Every $\epsilon$-isometry $f: E \rightarrow F$ between normed finite-dimensional vector spaces is $O(\epsilon)$-affine (affine up to an additive error which is a multiple of $\epsilon$ ).

Non-Proof Let $a, b \in E$ be arbitrary, and $z:=(a+b) / 2$. For any $\delta>0$ define $W_{\delta}$ to be the family of all $\epsilon$-isometries $g$, such that $d(g(a), a) \leq \delta$ and $d(g(b), b) \leq \delta$. Let $\lambda_{\delta}:=\sup \left\{\|g(z)-z\|: g \in W_{\delta}\right\}$. Similar to the original proof, we have

$$
d(\|g(z)-a\|,\|z-a\|) \leq 2 \delta
$$

and

$$
\|g(z)-z\| \leq\|g(z)-a\|+\|a-z\| \leq 2\|a-z\|+2 \delta .
$$

Again, this implies $\lambda_{\delta}$ is finite, with a bound which depends on $\|a-b\|$. However, this would not suffice for the final conclusion, as we have to apply this for all $a, b \in E$; what we need to show is that $\lambda_{\delta}$ has a bound indepent of $\|a-b\|$. 
Let $\psi$ be the reflection $x \mapsto 2 z-x$ of $E$ in $z$. For each $g \in W_{\delta}$ with rough inverse $g^{\prime} \in W_{\delta}$ holds $g^{*}:=\psi g^{\prime} \psi g \in W_{3 \delta}$, as $\|g(a)-a\| \leq \delta,\|(\psi g)(a)-b\| \leq \delta$, $\left\|\left(g^{\prime} \psi g\right)(a)-b\right\| \leq 3 \delta,\left\|\left(\psi g^{\prime} \psi g\right)(a)-a\right\| \leq 3 \delta$ (same for $b$ ). In contrast to $g$ and $g^{*}, \psi$ still is an isometry, with fixed point $z,\|\psi(x)-z\|=\|x-z\|$, and $\|\psi(x)-x\|=2\|x-z\|$.

$g$ and its rough inverse $g^{\prime}$ are $\delta$-isometries, so we find

$$
\begin{aligned}
2\|g(z)-z\| & =\|\psi(g(z))-g(z)\| \\
& \leq\left\|\left(g^{\prime} \psi g\right)(z)-z\right\|+2 \delta \\
& =\left\|\left(\psi g^{\prime} \psi g\right)(z)-z\right\|+2 \delta \\
& =\left\|g^{*}(z)-z\right\|+2 \delta \leq \lambda_{3 \delta}+2 \delta
\end{aligned}
$$

Choosing an appropriate sequence of $g_{j} \in W_{\delta}$, and including the already known bound for $\lambda$, we find the following two restrictions:

$$
\lambda_{\delta} \leq A+2 \delta \quad \text { and } \quad \lambda_{\delta} \leq \frac{1}{2} \lambda_{3 \delta}+\delta
$$

with $A:=2\|a-z\|=\|a-b\|$.

We now give an example to show that these restrictions are not strong enough to prove that $\lambda_{\delta}$ has an upper bound independent of $A$ : Set

$$
\lambda_{\delta}(A):=2 \cdot \sqrt[4]{A \cdot \delta^{3}} .
$$

Then due to $(\sqrt[4]{A \delta}-\sqrt{\delta})^{2} \geq 0$ and $(\sqrt{A}-\sqrt{\delta})^{2} \geq 0$ we have

$$
\begin{aligned}
\lambda_{\delta}(A) & \leq \sqrt{A \delta}+\delta \\
& \leq \frac{1}{2} A+\frac{3}{2} \delta \leq A+2 \delta .
\end{aligned}
$$

On the other hand, $\lambda_{3 \delta}=\lambda_{\delta} \cdot \sqrt[4]{27} \geq \lambda_{\delta} \cdot \sqrt[4]{16} \geq 2 \lambda_{\delta}$.

The first equations and inequalities of our "proof" started out well. It began to wallow just in the moment we introduced $W_{\delta}$ : We categorized certain isometries, and in contrast to the prior proof, it was not possible to categorize $g^{*}$ the same way, it landed in $W_{3 \delta}$. Why did we have to use $W_{\delta}$ the way we defined above? In the original proof, the connection to the isometry $f$ was that $h:=\psi f^{-1} \psi^{\prime} f$ would be in $W$. In our case, with $f$ an $\epsilon$-isometry, $h$ would be a $2 \epsilon$-isometry, and hence we had to define $W$ in one of two ways: Either the way we chose above (and failed), or to allow any rough isometry which approximately fixes $a$ and $b$ to enter $W$. This choice would have broken down the moment we try to prove that $\lambda$ is finite, as $\delta$ might have been arbitrarily large.

In retrospective, it seems plausible that the second-order-logic Väisälä applied is the obstacle against roughification of the proof, and that it might be possible to prove that any first-order-logic proof is stable against rough perturbations. 


\subsubsection{Rough Abelianness}

Apart from functions and theorems, it is also possible to replace axioms by their rough counterparts. We only want to touch upon this theme by giving a short categorization for rough abelianness.

Example 122

Let $\Gamma$ be some finitely generated group, $d$ some word metric on $G$ and $\epsilon>0$ fixed. Let us assume furthermore, that for all $g, h \in \Gamma$ holds:

$$
d(g h, h g) \leq \epsilon .
$$

As $\Gamma$ is finitely generated, we have: $d(g h, h g) \leq \epsilon$ for all $g, h \in \Gamma$ for some $\epsilon>0$ if and only if the set of commutators in $\Gamma$ is finite. According to [Ba], this is in turn equivalent to the commutator group $[G, G]$ being finite, and hence the abelianized group $G_{\mathrm{ab}}=G /[G, G]$ being of finite index. Our forthcoming Propositions 134 and 135 will then yield a natural $\delta$-isometry between $G$ and $G_{\mathrm{ab}}$ with $\delta=1+\operatorname{diam}([G, G])$. Note that the diameter of $[G, G]$ might be larger than $\epsilon$.

Conclusion: To be abelian is a roughly stable property; each roughly abelian group is roughly isometric to an abelian group (its abelianization).

\subsection{Coarse Relations for Groups}

When one speaks about the coarse geometry of finitely generated groups, one generally means quasi-isometries of Cayley graphs. While a single infinite group gives rise to an infinite number of non-isomorphic Cayley graphs, quasiisometries do not depend on the generating system of the group, and hence the quasi-isometry class of a group is well-defined, and an important invariant. It encompasses the idea of two groups being approximately isomorphic, but quasiisometries are not the only way to do this. Particularly the pure group-theoretic notion of commensurability rivals the quasi-isometry, and their interplay is still an interesting research problem. In the following, we use the definitions given in $\mathrm{dH}$.

Definition 123

Let $G$ and $H$ be groups. $G$ and $H$ are commensurable when there exist subgroups $G^{\prime} \leq G$ and $H^{\prime} \leq H$ of finite index, such that $G^{\prime}$ and $H^{\prime}$ are isomorphic as group.

$G$ and $H$ are commensurable up to finite kernels if there exists a finite sequence of groups $\Gamma_{1}, \ldots, \Gamma_{N}$ and homomorphisms $h_{0}, \ldots, h_{N}$

$$
G \stackrel{h_{0}}{\longrightarrow} \Gamma_{1} \stackrel{h_{1}}{\longleftarrow} \Gamma_{2} \stackrel{h_{2}}{\longrightarrow} \Gamma_{3} \stackrel{h_{3}}{\longleftarrow} \ldots \stackrel{h_{N-1}}{\longrightarrow} \Gamma_{N} \stackrel{h_{N}}{\longleftarrow} H
$$

with finite kernels and images of finite index. 
One easily sees that commensurability always implies commensurability up to finite kernels, which in turn always implies quasi-isometry, given that both groups are finitely generated. We quote without proof the following Proposition from $[\mathrm{dH}, \mathrm{IV} .28$.

Proposition 124

Two residually finite groups are commensurable if and only if they are commensurable up to finite kernels.

Proposition 125

Let $G$ and $H$ be f.g. groups, and $\eta: G \rightarrow H$ a homomorphism and quasiisometry. Then $G$ and $H$ are commensurable up to finite kernels.

Proof The kernel of $\phi$ is finite, because it is the preimage of a finite subset of $H$. And the image $\phi(G)$ is a subgroup of $H$ of finite index: $\phi(G)$ is $\epsilon$-dense in $H$. Let $B$ be the $\epsilon$-ball around the identity in $H$, then each element $h \in H$ can be written as $b \cdot \phi(g)$ for some $b \in B$ and $g \in G$. With this, the number of cosets of $G / \phi(g)$ can be at most as large as \#B, and in particular, it is finite.

There is a multitude of cases in which quasi-isometry implies commensurability (for example f.g. abelian groups, certain types of Baumslag-Solitar groups, abelian-by-cyclic groups in [FM]) but also a plenty supply of counter-examples (e.g. Lamplighter groups, or $\mathbb{Z}^{2} \rtimes_{A} \mathbb{Z}$ with certain choices for $A \in \operatorname{GL}(2, \mathbb{Z})$ ).

We want to add to this discussion by defining new kinds of coarse equivalences for f.g. groups, situated between commensurability and quasi-isometry, and mostly based upon rough isometries of Cayley graphs. Rough isometries are stronger than quasi-isometries, and hence we might expect equivalence to commensurability for larger classes of groups. However, rough isometries are not a canonical notion for groups, as they depend on the chosen generating set. This problem unfolds into a rich zoo of different notions of rough isometry for groups.

Example 126

The weakest of these notions motivates a theorem of the form:

Groups are commensurable if and only if they admit generating systems such that their Cayley graphs are roughly isometric.

Unfortunately, this is wrong, the property is too weak. The counter-example are the lamplighter groups (see [dH] IV.44)

$$
\Gamma_{F}:=\left(\bigoplus_{j \in \mathbb{Z}} F_{j}\right) \rtimes \mathbb{Z}
$$

where $\mathbb{Z}$ acts by shifting, and each $F_{j}$ is a copy of a finite group $F$. For finite groups $F, G$ of same size there are generating systems of $\Gamma_{F}$ and $\Gamma_{G}$ such that 
the corresponding Cayley graphs are even isomorphic as graphs. Now one may choose $F$ solvable and $G$ not. Then $\Gamma_{F}$ will be solvable, but $\Gamma_{G}$ not virtually solvable, which implies that they cannot be commensurable. This is the classic example to show that quasi-isometry does not imply commensurability, and it works for rough isometries (and even isometries) equally well.

A related question is whether two isomorphic Cayleygraphs imply that their groups are isomorphic. This is in general not the case (as long as only one Cayleygraph per group is considered), and is already in the finite case a rich source for problems, see $[\mathrm{L}$.

We are not yet able to categorize all of these notions and give proofs or counterexamples to their mutual equivalences. However, we will take a closer look at two of these definitions. Our methods involve the analysis of generating systems and groups of what we call "shared isometries" and "shared rough isometries" - maps which are isometries (respectively rough isometries) relative to lots of generating systems at once. But before we get there, we insert a section about a rough isometry invariant, and another section about the case in abelian groups. These two sections together provide first insights.

\subsection{Exponential Growth Rate}

Each quasi-isometry invariant is also a rough-isometry invariant. But there also is a rough isometry invariant, which is not a quasi-isometry invariant:

Definition 127

127

Let $G$ be a f.g. group, and let $S$ be a finite generating system of $G$. The exponential growth rate is

$$
\omega(G, S):=\limsup _{k \rightarrow \infty} \sqrt[k]{\# B_{G, S}(k)}=\exp \limsup _{k \rightarrow \infty} \frac{\ln \# B_{G, S}(k)}{k} .
$$

The minimal growth rate $\omega(G)$ is the infimum of $\omega(G, S)$ over all finite generating systems $S$. The group $G$ is of uniformly exponential growth if $\omega(G)>1$.

Proposition 128

Let $F_{n}$ be the free group on $n$ generators. Then $\omega\left(F_{n}\right)=2 n-1$.

Proof This is Proposition VII.12 in $\mathrm{dH}$, we summarize the proof here: The minimal growth rate is attained by any free generating system for $F_{n}$. Now let $S$ be any generating system of $F_{n}$. Let $S^{\prime}$ be the image of $S$ under abelianization, choose a minimal subset of $S^{\prime}$ generating a finite index subset of $\mathbb{Z}^{n}$. Any preimage of $S^{\prime}$ is a set of free generators of a subgroup $H$ of $F_{n}$, which in turn is isomorphic to $F_{n}$ and of growth $2 n-1$. Hence, $\omega\left(F_{n}, S\right) \geq \omega\left(H, S^{\prime}\right)=2 n-1$. 
Lemma 129

Let $G, H$ be f.g. groups of uniformly exponential growth, let $S_{G}$ and $S_{H}$ be finite generating systems of $G$ and $H$, and let

$$
\eta: \operatorname{Cay}\left(G, S_{G}\right) \rightarrow \operatorname{Cay}\left(H, S_{H}\right)
$$

be an $\epsilon$-isometry, $\epsilon \geq 0$. Then $\omega\left(G, S_{G}\right)=\omega\left(H, S_{H}\right)$.

Proof By estimating the number of elements in each ball:

$$
\begin{aligned}
\frac{\# B_{G}(r)}{\# B_{G}(\epsilon)} & \leq \# \eta\left(B_{G}(r)\right) \\
& \leq \# B_{H}(r+\epsilon) \\
& \leq \# B_{H}(r) \cdot \# B_{H}(\epsilon) \\
\Rightarrow \omega\left(G, S_{G}\right) & \leq \omega\left(H, S_{H}\right),
\end{aligned}
$$

and vice versa.

Example 130

Although the free groups $F_{2}$ and $F_{4}$ are commensurable, there is a generating system of $F_{2}$, such that its Cayley graph is not roughly isometric to any Cayley graph of $F_{4}$, as the minimal growth rates differ.

However, there still exist generating systems of $F_{2}$ and $F_{4}$ with their Cayley graphs being roughly isometric: Choose any embedding $\pi$ of $F_{4}$ into $F_{2}$ as subgroup of finite index, let $S_{j}$ be free generating systems of $F_{j}, j=2,4$. Choose $S:=S_{2} \cup \pi\left(S_{4}\right)$ as generating system for $F_{2}$, then due to the uniqueness of each word in $F_{2}$ and due to the corresponding unique length function, $\pi$ is a rough isometry between $\operatorname{Cay}\left(F_{2}, S\right)$ and $\operatorname{Cay}\left(F_{4}, S_{4}\right)$.

\subsection{The Abelian Case}

Lemma 131

Let $N$ be a finite subgroup of $G L(n, \mathbb{Z}), n \in \mathbb{N}^{*}, G_{0}=\mathbb{Z}^{n}$, and $G=G_{0} \rtimes$ $N$ with canonical action. Then for each generating system $S$ of $G$ there is a generating system $S^{\prime}$ of $\mathbb{Z}^{n}$, such that the canonical embedding $i: \mathbb{Z}^{n} \hookrightarrow G$ is a rough isometry of $\mathrm{Cay}\left(\mathbb{Z}^{n}, S^{\prime}\right)$ and $\mathrm{Cay}(G, S)$.

Proof Choose

$$
S^{\prime}:=\left\{g^{-1} s g \mid g \in N, s \in S\right\} .
$$

Let $d^{\prime}$ be the metric in Cay $\left(\mathbb{Z}^{n}, S^{\prime}\right)$, and $d$ the metric in $\operatorname{Cay}(G, S)$. Let $x \in G_{0}$ be arbitrary. As $S \subseteq S^{\prime}$, we obviously have $d^{\prime}(0, x) \leq d(0, x)$. Now represent $x$ in $S^{\prime}$ :

$$
x=s_{1}^{g_{1}} \cdot s_{2}^{g_{2}} \cdot \ldots \cdot s_{k}^{g_{k}}
$$



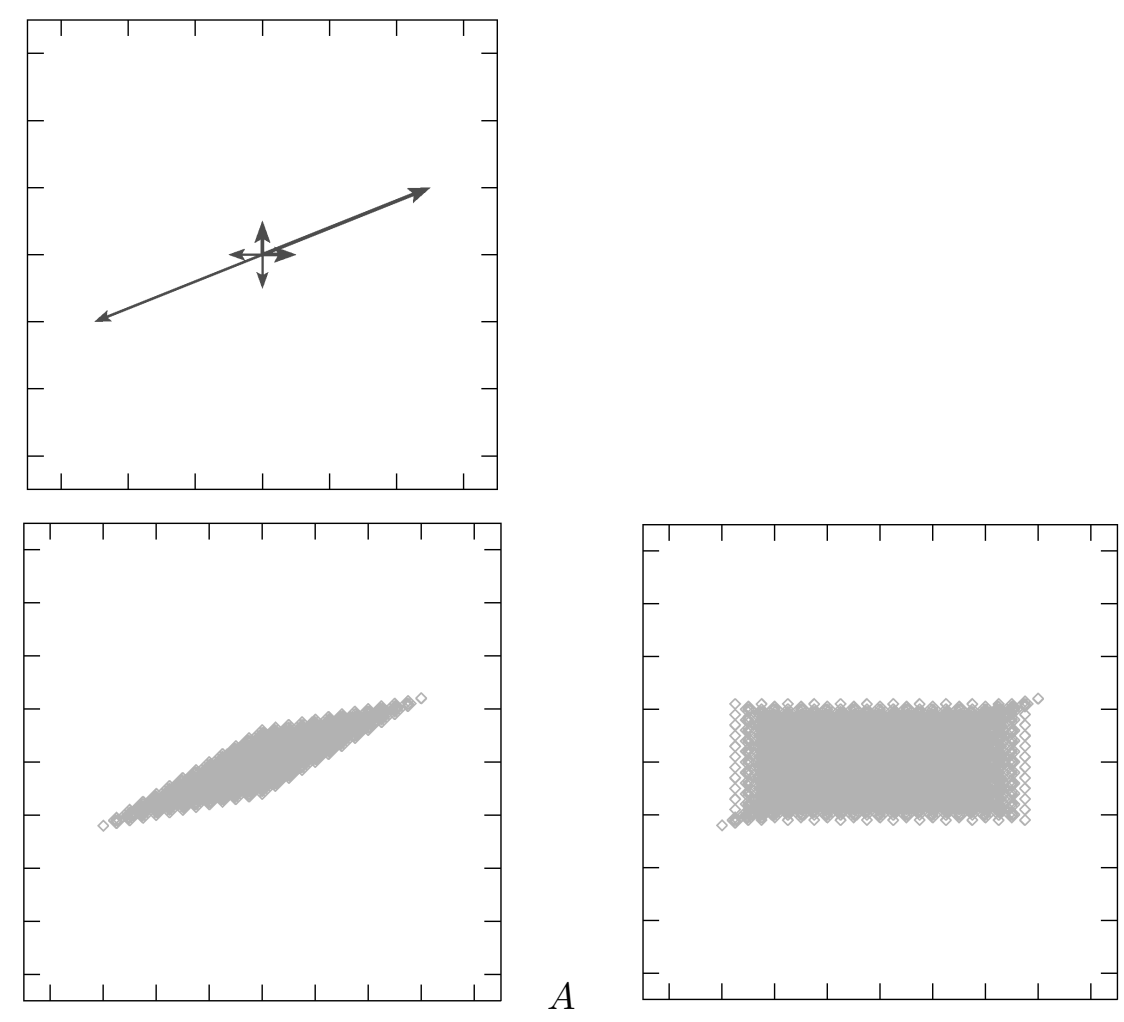

$B$
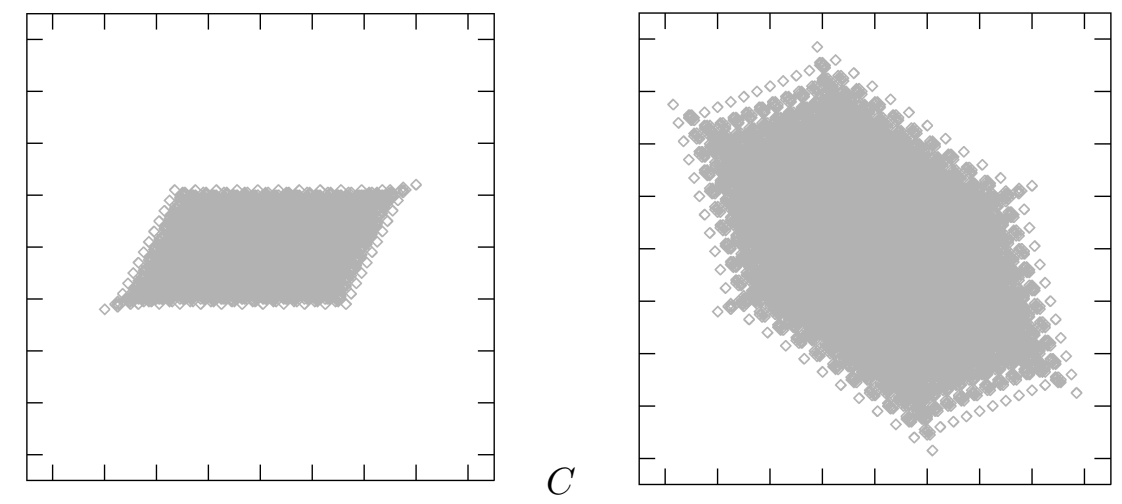

$D$

$A=\left(\begin{array}{ll}1 & 0 \\ 0 & 1\end{array}\right) \quad B=\left(\begin{array}{cc}1 & 0 \\ 0 & -1\end{array}\right) \quad C=\left(\begin{array}{ll}1 & -1 \\ 0 & -1\end{array}\right) \quad D=\left(\begin{array}{cc}0 & 1 \\ -1 & -1\end{array}\right)$

Figure 3.1: A collection of balls of radius 12 in various groups of kind $G=\mathbb{Z}^{2} \rtimes\langle M\rangle$, with $M \in \mathrm{GL}(2, \mathbb{Z})$, projected on the canonical subgroup $\mathbb{Z}^{2}$. All four of these Cayleygraphs are constructed from the same generating system, which is depicted in the upper image. While the trivial case $A$ is the expected convex span of the generating system (approximately, and modulo the discrete structure of $\mathbb{Z}^{2}$ ), non-trivial matrices generate larger balls by enforcing approximate symmetries by their action: If $w$ is a word in $\mathbb{Z}^{2}$ of length $L$, then $M w$ is of length $\leq L+1$ in $G$. 
with $g_{j} \in N, s_{j} \in S$, and $g_{1} g_{2} \ldots g_{k}=e$, because $x \in G_{0}$. Then by commutativity we can rearrange the word to collect all instances of an element of $N$ :

$$
x=\prod_{g \in N}\left(s_{g, 1} s_{g, 2} \ldots s_{g, l(g)}\right)^{g}
$$

with $\sum l(g)=k$. Represent each of the finitely many $g \in N$ with a minimal word $w_{g}$ of letters in $S$. Let $L$ be the greatest length among the $w_{g}$, then $x$ is of length $\leq k+\epsilon$ with $\epsilon=2 \cdot \# N \cdot L$. Hence $i$ is an $\epsilon$-isometric embedding.

Now let $x \in G$ be arbitrary. As $N$ is a normal subgroup, there is $g \in N$ and $h \in G_{0}$ such that $x=g h$. The element $g$ is of length $\leq L$ in $S$, hence $G_{0}$ is $L$-dense in $G$, and $i$ is an $\epsilon$-isometry.

Corollary 132 132

Let $G=\mathbb{Z}^{n} \rtimes N$ be generated by a generating system $S^{\prime}$ of $\mathbb{Z}^{n}$ and the whole of $N \backslash\{e\}$. Let $r \in \mathbb{N}^{*}$ be arbitrary, then the $r$-ball in $G$ is at bounded Hausdorffdistance to the set $A \subseteq \mathbb{Z}^{n}$ which is constructed in the following way:

1. Take $A_{1}$ to be the r-ball of $S^{\prime}$ in $\mathbb{Z}^{n}$,

2. $A_{2}$ the canonical embedding of $A_{1}$ into $\mathbb{R}^{n}$,

3. $A_{3}$ the union of the orbit of $A_{2}$ under $N$,

4. $A_{4}$ the convex hull of $A_{3}$,

5. and finally $A=A_{4} \cap \mathbb{Z}^{n}$.

We may restrict any word metric on $G$ to its subgroup $\mathbb{Z}^{n}$ and visually compare the possible geometries by comparing their generated unit balls in $\mathbb{Z}^{n}, N$ will then impose symmetries on the possible geometries. This can be seen in Figure 3.1 for the case $n=2$ and three choices of cyclic subgroups of $\mathrm{GL}(2, \mathbb{Z})$.

While the quasi-isometry and commensurability classes of a group are given by any (normal) subgroup of finite index, the finite quotients can still modulate the possible rough isometry classes of a group:

- In the above case of semidirect products of abelian groups, they simply restrict to metrics suitable for the corresponding symmetries: The group has less or equally many rough isometry classes than its subgroups.

- In the case of free groups in section 3.3 the finite quotients may as well increase the number of possible metrics, as the exponential growth rate shows $\left(F_{2}\right.$ allows metrics which cannot be generated by its finite-index subgroup $\left.F_{4}\right)$ : Here, the group has more or equally many rough isometry classes than its subgroups. 
Example 133

We now give an example that the relation

$$
\begin{aligned}
G \sim H: \Leftrightarrow \quad \exists S_{G}, S_{H} \text { generating systems, such that } \operatorname{Cay}\left(G, S_{G}\right) \\
\\
\text { and Cay }\left(H, S_{H}\right) \text { are roughly isometric }
\end{aligned}
$$

is not an equivalence relation per se. We choose

$$
G:=\mathbb{Z}^{2} \rtimes\left(\begin{array}{cc}
0 & 1 \\
-1 & 1
\end{array}\right) \quad \text { and } \quad H:=\mathbb{Z}^{2} \rtimes\left(\begin{array}{cc}
0 & 1 \\
-1 & 0
\end{array}\right) .
$$

By Lemma 131 we have $G \sim \mathbb{Z}^{2} \sim H$. Assume there are generating systems $S_{G}$ and $S_{H}$ such that $\operatorname{Cay}\left(G, S_{G}\right)$ and $\operatorname{Cay}\left(H, S_{H}\right)$ are roughly isometric. Each $r$-ball must (approximately) adhere to both symmetry groups. The order of the first one is 6 , the order of the second is 4 , but there is no finite subgroup of $\mathrm{GL}(2, \mathbb{Z})$ of order $\operatorname{lcm}(6,4)=12$ or higher.

\subsection{Rough Isometries of Quotients with Finite Kernel}

\section{Proposition 134}

134

Let $G$ be a f.g. group, let $H \unlhd G$ be a finite normal subgroup, and set $G^{\prime}:=$ $G / H$. Then each finite generating system $S_{0}$ of $G$ can be enlarged to a finite generating system $S$ of $G$, such that $\eta: G \rightarrow G^{\prime}, g \mapsto g H$ is a 1-isometry, where $G^{\prime}$ is endowed with the word metric of the projection $S^{\prime}$ of $S$.

Proof Let $S_{0}$ be some generating set of $G$, and put $S:=S_{0} \cup H \backslash\{e\}$. Define $S^{\prime}:=\{s H: s \in S\} \backslash\{e\}$ as the non-trivial cosets of $S$. $S^{\prime}$ generates $G^{\prime}$ : For each $x \in G^{\prime}$ is $x=g H$ for some $g \in G$, present $g$ as $s_{1} \ldots s_{n}$ with $s_{j} \in S_{0}$. Then $x=\left(s_{1} H\right) \cdot \ldots \cdot\left(s_{n} H\right)$. From this we see $d^{\prime}(e H, g H) \leq d(e, g)$ with $d$ the word metric resulting from $S \subseteq G$ and $d^{\prime}$ the word metric for $S^{\prime} \subseteq G^{\prime}$.

On the other hand, let $g \in G$ be arbitrary, and let $g H=\left(s_{1} H\right) \cdot \ldots \cdot\left(s_{n} H\right) \in G^{\prime}$ be a shortest word in $G^{\prime}$. Then there is $h \in H$ with $g=s_{1} \ldots s_{n} \cdot h$, hence $d(e, g) \leq d^{\prime}(e H, g H)+1$. Finally, let $x \in G^{\prime}$ be any coset. Choose any representative $g$ of this coset, thus $g H=x$. Then $d(x, \eta(g))=d(x, g H)=0$.

Note that in the preceding proof we might have chosen some generating set $S_{H}$ of $H$ and set $S:=S_{0} \cup S_{H}$. In this case, the proof would yield an $\epsilon$-isometry with $\epsilon=\operatorname{diam} \operatorname{Cay}\left(H, S_{H}\right)$ instead.

Let $S$ be some generating set of the finitely generated group $G, H \unlhd G$ finite, and $S_{H}$ a generating set of $H$. Then the identity $\left(G, d_{S}\right) \rightarrow\left(G, d_{S \cup S_{H}}\right)$ is an $\epsilon$-isometry with $\epsilon \leq \operatorname{diam}_{S}(H)$. 
Proof Let $d:=d_{S}, d^{\prime}:=d_{S} \cup H$. We obviously have $d^{\prime}(e, g) \leq d_{S} \cup S_{H}(e, g) \leq$ $d(e, g)$ for all $g \in G$. Now let $g=s_{1} t_{1} \ldots s_{n} t_{n}$ be some presentation of $g \in G$ in generators $s_{j} \in S \cup\{e\}$ and $t_{j} \in H$. As $H$ is normal, we can find $t_{1}^{\prime}$ to $t_{n}^{\prime} \in H$ with $g=s_{1} \ldots s_{n} \cdot t_{1}^{\prime} \ldots t_{n}^{\prime}$. Hence $d(e, g) \leq d^{\prime}(e, g)+\epsilon$ where $\epsilon$ is the diameter of $H \subseteq G$ in $d_{S}$.

\subsection{Shared Isometries}

Definition 136

Consider $\epsilon \geq 0$, and let $G$ be a finitely generated group. Let $\mathfrak{S}$ be a family of generating systems. Define the $\mathfrak{S}$-shared or simply shared isometry groups and sets

$$
\begin{aligned}
(\lambda, \epsilon)-\operatorname{Isom}_{\mathfrak{S}}(G) & :=\{\eta: G \rightarrow G \mid \forall S \in \mathfrak{S}: \eta \text { is a }(\lambda, \epsilon) \text {-qi. rel. to } S\} \\
\epsilon-\operatorname{Isom}_{\mathfrak{S}}(G) & :=(1, \epsilon)-\operatorname{Isom}_{\mathfrak{S}}(G) \\
\operatorname{Isom}_{\mathfrak{S}}(G) & :=0-\operatorname{Isom}_{\mathfrak{S}}(G) \\
\operatorname{UQIsom}_{\mathfrak{S}}(G) & :=\bigcup_{\lambda, \epsilon \geq 0}(\lambda, \epsilon)-\operatorname{Isom}_{\mathfrak{S}}(G) \\
\operatorname{URIsom}_{\mathfrak{S}}(G) & :=\bigcup_{\epsilon \geq 0} \epsilon-\operatorname{Isom}_{\mathfrak{S}}(G)
\end{aligned}
$$

The last ones we call $\mathfrak{S}$-uniform quasi-isometries resp. rough isometries. We further define

$$
\begin{aligned}
\epsilon-\operatorname{Iden}_{\mathfrak{S}}(G) & :=\{\eta: G \rightarrow G \mid \forall S \in \mathfrak{S}: \eta \text { is } \epsilon \text {-near the identity }\} \\
\operatorname{Iden}_{\mathfrak{S}}(G) & :=\bigcup_{\epsilon \geq 0} \epsilon-\operatorname{Iden}_{\mathfrak{S}}(G) .
\end{aligned}
$$

These definitions are similar to the definition of the quasi-isometry group QI of a true metric space or group (the calculation of QI is very difficult in general, see for example [FM]), and we find composition to be a group structure on $\operatorname{UQIsom}_{\mathfrak{S}}(G)$ and on $\operatorname{URIsom}_{\mathfrak{S}}(G)$ after quotiening out $\operatorname{Iden}_{\mathfrak{S}}(G)$. The difference between the quasi-isometry group QI $(G)$ and $\operatorname{UQIsom}_{\mathfrak{S}}(G) / \operatorname{Iden}_{\mathfrak{S}}(G)$ seems to be subtle, as we just demand $\lambda$ and $\epsilon$ to be uniformly bounded for all word metrics in $\mathfrak{S}$, but this difference can be enormous, if $\mathfrak{S}$ is chosen large enough. On the other hand, if $\mathfrak{S}$ comprises only a finite number of generating systems, $\mathrm{UQIsom}_{\mathfrak{S}}(G) / \operatorname{Iden}_{\mathfrak{S}}(G)$ equals $\mathrm{QI}(G)$, independently of the exact choice of $\mathfrak{S}$. We will begin with the examination of $\operatorname{UQIsom}_{\mathfrak{S}}(G)$ and $\operatorname{URIsom}_{\mathfrak{S}}(G)$ in Section 3.7 and now concentrate on the nearly trivial case of $\operatorname{Isom}_{\mathfrak{S}}(G)$. We start with a simple observation, which resulted from a discussion with Laurent Bartholdi and Martin Bridson during the 2007 winter school "Geometric Group Theory" in Göttingen:

Theorem 137 (L. Bartholdi '07)

(A) Let $\mathfrak{S}=\mathfrak{S}_{\text {asym }}$ be the family of all, possibly asymmetric, finite generating 
systems of $G$. Then $\operatorname{Isom}_{\mathfrak{S}}(G)$ is isomorphic to $G$ (using possibly asymmetric distance functions).

(B) Let $G$ be a group with a finite, symmetric generating system $S_{0}$ such that the following hold:

1. There are no $s_{1}, s_{2}, s_{3} \in S_{0}$ with $s_{1} s_{2}=s_{3}$. (Minimality; easy to achieve.)

2. There are no $s_{1}, s_{2} \in S_{0}, s_{1} \neq s_{2}^{ \pm 1}$, with $s_{1}^{2} s_{2}^{2}=e$.

3. There are no $s_{1}, s_{2} \in S_{0}, s_{1} \neq s_{2}^{ \pm 1}$, with $s_{1}^{s_{2}}=s_{1}^{-1}$.

4. There are no $s_{1}, s_{2} \in S_{0}, s_{1} \neq s_{2}^{ \pm 1}$, with $s_{1}^{s_{2}}=s_{1}$ (In particular, $G$ is not an abelian group.)

5. There are at least two distinct elements in $S_{0}$, which are not inverses of each other.

Let $\mathfrak{S}=\mathfrak{S}_{\text {sym }}$ be the family of all symmetric finite generating systems of $G$. Then $\operatorname{Isom}_{\mathfrak{S}}(G)$ is isomorphic to $G$.

(C) Let $G$ be a f.g. abelian group without 2-torsion, and let $\mathfrak{S}=\mathfrak{S}_{\mathrm{sym}}$ be the family of all symmetric finite generating systems of $G$. Then $\operatorname{Isom}_{\mathfrak{S}}(G)$ is isomorphic to $G \rtimes C_{2}$, where $C_{2}$ acts by inversion $x \mapsto x^{-1}$.

(D) Let $G$ be a f.g. group, and $S_{0} \in \mathfrak{S}=\mathfrak{S}_{\text {sym }}(G)$, such that $S_{0}$ is minimal, and each element $s \in S$ has order 2 (i.e. $s^{2}=e$ ). Then $\operatorname{Isom}_{\mathfrak{S}}(G) \cong G$.

Proof (A) Consider $\phi \in \operatorname{Isom}_{\mathfrak{S}}(G)$, and $x, s \in G$ arbitrary, $s \neq e$. Let $\mathfrak{S}^{\prime}:=$ $\{S \in \mathfrak{S}: s \in S\}$. Then $d_{S}(x, x s)=1$ and $d_{S}(\phi(x), \phi(x s))=1$ for each $S \in \mathfrak{S}^{\prime}$, i.e. $s_{x}:=\phi(x)^{-1} \cdot \phi(x s) \in S$. Assume $s_{x} \neq s$. Then define $S^{\prime}:=\left(S \backslash\left\{s_{x}\right\}\right) \cup$ $\left\{s, s^{-1} s_{x}\right\} . S^{\prime}$ is again a generating system and $s_{x} \notin S^{\prime}$, as $s \neq s_{x}$ and $s \neq e$. Yet, we have $s \in S^{\prime}$, contradiction. So we conclude $s_{x}=s$ and $\phi(x s)=\phi(x) \cdot s$. By induction we find $\phi(x)=\phi(e) \cdot x$, with $\phi(e)$ arbitrary. On the other hand, each such $\phi$ obviously is in $\operatorname{Isom}_{\mathfrak{S}}(G)$, and

$$
G \ni g \mapsto\left(\phi_{g}: x \mapsto g \cdot x\right) \in \operatorname{Isom}_{\mathfrak{S}}(G)
$$

are shared isometries, and $\phi_{g} \circ \phi_{h}=\phi_{g h}$.

(B) Let $\phi \in \operatorname{Isom}_{\mathfrak{S}}(G)$, and $x \in G$ arbitrary, $s \in S_{0}$. Then $d_{S_{0}}(x, x s)=1$ and $d_{S_{0}}(\phi(x), \phi(x s))=1$, i.e. $s_{x}:=\phi(x)^{-1} \cdot \phi(x s) \in S_{0}$. Like in the asymmetric case, using $\mathfrak{S}^{\prime}:=\{S \in \mathfrak{S}: s \in S\} \ni S_{0}$ we find $s_{x}=s$ or $s_{x}=s^{-1}$, but the choice might depend on $x$, and this is the main point differing to the asymmetric case. Now let $r \in S_{0}$ be arbitrary, $r \neq s^{ \pm 1}$ and $S_{0}^{\prime}:=S_{0} \cup\left\{s r,(s r)^{-1}\right\}$. Note that $d_{S_{0}}(x, x s r)=2$, as there are no triangles in $S_{0}$, but $d_{S_{0}^{\prime}}(x, x s r)=1$. Let $r_{y}=$ $\phi(y)^{-1} \cdot \phi(y r) \in S_{0}$, so we find $\phi(x s r)=\phi(x) \cdot s_{x} \cdot r_{x s}$. As $d_{S_{0}^{\prime}}(\phi(x), \phi(x s r))=1$, we have

1. $s_{x}=s$ or $s_{x}=s^{-1}$,

2. $r_{x s}=r$ or $r_{x s}=r^{-1}$, 
3. $s_{x} r_{x s} \in S_{0}^{\prime}$, but $s_{x} r_{x s} \notin S_{0}$.

Hence, $s_{x} r_{x s}$ must be one of the added elements $s r$ or $(s r)^{-1}=r^{-1} s^{-1}$. We find eight cases:

1. $s_{x}=s, r_{x s}=r, s_{x} r_{x s}=s r$

2. $s_{x}=s^{-1}, r_{x s}=r, s_{x} r_{x s}=s r \quad \Rightarrow \quad s^{2}=e \quad \curvearrowright \quad$ case (1)

3. $s_{x}=s, r_{x s}=r^{-1}, s_{x} r_{x s}=s r \quad \Rightarrow \quad r^{2}=e \quad \curvearrowright \quad$ case (1)

4. $s_{x}=s^{-1}, r_{x s}=r^{-1}, s_{x} r_{x s}=s r \quad \Rightarrow \quad s^{2} r^{2}=e$

5. $s_{x}=s, r_{x s}=r, s_{x} r_{x s}=r^{-1} s^{-1} \quad \Rightarrow \quad(s r)^{2}=e \quad \curvearrowright \quad$ case (1)

6. $s_{x}=s^{-1}, r_{x s}=r, s_{x} r_{x s}=r^{-1} s^{-1} \Rightarrow r^{s}=r^{-1}$

7. $s_{x}=s, r_{x s}=r^{-1}, s_{x} r_{x s}=r^{-1} s^{-1} \Rightarrow s^{r}=s^{-1}$

8. $s_{x}=s^{-1}, r_{x s}=r^{-1}, s_{x} r_{x s}=r^{-1} s^{-1} \Rightarrow r^{s}=r$

Cases (2), (3) and (5) directly lead to case (1) after re-inserting, case (4) contradicts property (2) for $S_{0}$, cases (6), (7) and (8) contradict properties (3) and (4). Hence, we are left with case (1), and $s_{x}=s$ for all $x \in G$. Again, we use induction to show $\phi(x)=\phi(e) \cdot x$, and get an isomorphism

$$
G \ni g \mapsto\left(\phi_{g}: x \mapsto g \cdot x\right) \in \operatorname{Isom}_{\mathfrak{S}}(G)
$$

(C) It is easy to find a generating system $S_{0}$ of $G$ which fulfills all properties of subtheorem (B), except for property (4): $s_{1}^{s_{2}}=s_{1}$ is always true. We follow through the proof of subtheorem (B) until case (8) cannot be contradicted. Assume it is realized, i.e. we find $x \in G, s \in S_{0}$ with $\phi(x s)=\phi(x) \cdot s^{-1}$. Then, for each $r \in S \backslash\left\{s, s^{-1}\right\}$ we must have $\phi(x s r)=\phi(x) \cdot s^{-1} \cdot r^{-1}$, and from excluding all other cases and property (2) of $S_{0}$ we further find $\phi\left(x s^{2}\right)=\phi(x) \cdot s^{-2}$. By induction and using the fact that $S_{0}$ generates $G$, we show

$$
\phi\left(s_{1} s_{2} \ldots s_{n}\right)=\phi(e) \cdot s_{1}^{-1} s_{2}^{-1} \ldots s_{n}^{-1},
$$

or, due to abelianness, $\phi(x)=\phi(e) \cdot x^{-1}$. Obviously, all these bijections are indeed shared isometries:

$$
\begin{aligned}
d(\phi(x), \phi(y)) & =d\left(x^{-1}, y^{-1}\right)=\left\|x y^{-1}\right\| & & \mid \text { abelianness } \\
& =\left\|y^{-1} x\right\|=d(y, x) & & \mid S_{0} \text { is symmetric } \\
& =d(x, y) & &
\end{aligned}
$$

Hence, we have $\operatorname{Isom}_{\mathfrak{S}}(G)$ isomorphic to $G \rtimes C_{2}$ via

$$
G \rtimes C_{2} \ni(g, a) \mapsto\left(\phi_{(g, a)}: x \mapsto g \cdot x^{a}\right) \in \operatorname{Isom}_{\mathfrak{S}}(G)
$$

(D) Once again, we follow through the proof of subtheorem (B). As $S_{0}$ is minimal, property (1) is automatically fulfilled. And as each $s \in S_{0}$ has order 2, 
the question $s_{x}=s$ or $s_{x}=s^{-1}$ is trivial, as $s^{-1}=s$. Hence, we get the usual isomorphism

$$
G \ni g \mapsto\left(\phi_{g}: x \mapsto g \cdot x\right) \in \operatorname{Isom}_{\mathfrak{S}}(G)
$$

From now on, we will restrict to the symmetric case $\mathfrak{S}=\mathfrak{S}_{\text {sym }}$.

\section{Example 138}

For groups $G$ with central elements it can be difficult to find a generating system $S_{0}$ satisfying the properties of Theorem 137, B, but typically it is still possible. Take for example:

$$
\begin{aligned}
G & =\langle a, b, c \mid[a, c],[b, c]\rangle \cong(\mathbb{Z} * \mathbb{Z}) \times \mathbb{Z} \\
S_{0} & =\left\{a^{ \pm 1},(b c)^{ \pm 1},(a b)^{ \pm 1}\right\} .
\end{aligned}
$$

Example 139

The same accounts for groups with 2-torsion. For example, it is easy to calculate by hand

$$
\operatorname{Isom}_{\mathfrak{S}}\left(C_{2}\right) \cong C_{2}
$$

just as Theorem 137. D mentions; but not $C_{2} \rtimes C_{2}$, as one might think from Theorem 137. C. Indeed, as inversion is the trivial operation in each group of exponent 2, we have $\operatorname{Isom}_{\mathfrak{S}}\left(C_{2}\right)^{n} \cong\left(C_{2}\right)^{n}$ in the abelian case, contrary to Theorem 137 . $C$.

Example 140

For groups of the form $G=G_{0} \rtimes C_{2}$ with $C_{2}$ acting via inversion (written multiplicatively) on a f.g. group $G_{0}$ (which subsequently must be abelian), each element $(g,-1)$ with $g \in G_{0}$ has torsion 2. Given a minimal generating system $S_{0}$ of $G_{0}$, we can use

$$
S:=\left\{(g,-1): g \in S_{0}\right\} \cup\{(e,-1)\}
$$

to apply Theorem 137.D. And, just as it states, the inversion is not a shared isometry in this case: Let $G_{0}$ be any f.g. group with at least one element $s \in G_{0}$ with $s^{2} \neq e, S_{0}$ a finite generating system of $G_{0}$ with $s \in S_{0}$, and $S^{\prime}:=S_{0} \cup$ $\{(s,-1)\}$, which generates $G=G_{0} \rtimes C_{2}$. Then holds $d((e,-1),(s, 1))=1$, as $(e,-1) \cdot(s,-1)=(s, 1)$, but

$$
\begin{array}{r}
d\left((e,-1)^{-1},(s, 1)^{-1}\right)=d\left((e,-1),\left(s^{-1}, 1\right)\right)>1, \\
\text { because }\left(s^{-1},-1\right) \notin S^{\prime} .\left(s \neq s^{-1} \text {, and }(s,-1)^{-1}=(s,-1) .\right)
\end{array}
$$




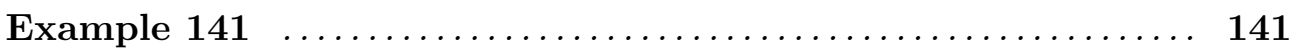

Similar to Example 140, consider a group $G=G_{0} \rtimes H$, where a f.g. group $H$ acts on the f.g. abelian group $G_{0}$. The action shall be given by a non-trivial homomorphism $\alpha: H \rightarrow C_{2}$, where $C_{2}$ acts on $G_{0}$ by inversion. Furthermore, let $S_{0}$ be an arbitrary finite generating system of $G_{0}$, and let $S_{H}$ be a finite generating system for $H$, such that there are no two elements $s, t \in S$ with $s \neq t^{ \pm 1}$ and $s^{t}=s^{ \pm 1}$ or $s^{2} t^{2}=e$. Finally, let $h_{0} \in S_{H}$ be an element with $h_{0}^{4} \neq e$. Then we can define a finite generating system

$$
S_{0}:=S_{H} \cup\left\{g h_{0}: g \in S_{0}\right\}
$$

from which we choose a minimal subsystem $S \subseteq S_{0}$. Some simple calculations then show that the generating system $S$ fulfills the requirements for Theorem 137. B, and we conclude:

$$
\operatorname{Isom}_{\mathfrak{S}}\left(G_{0} \rtimes H\right) \cong G_{0} \rtimes H
$$

In particular, this accounts for the group

$$
\mathbb{Z} \rtimes \mathbb{Z}=\left\langle x, y: x^{y}=x^{-1}\right\rangle \cong\left\langle y, z: y^{2}=z^{2}\right\rangle .
$$

Considering the proof of Theorem 137 and the above examples, we are confident that the following statements can be proven just by application of more arduous combinatorics:

(A) Let $G$ be a f.g. group, and let $\mathfrak{S}$ be the family of all symmetric generating systems of $G$. Then $\operatorname{Isom}_{\mathfrak{S}}(G) \cong G \rtimes C_{2}$ if and only if $G$ is non-trivial, abelian, and not of exponent 2 ; $\operatorname{Isom}_{\mathfrak{S}}(G) \cong G$ otherwise.

(B) The shared Clifford isometries (i.e. those shared isometries $\phi$ with constant $d(x, \phi(x))$ for all $x \in G)$ always constitute a group, which is isomorphic to $G$.

Lemma 142

Let $G, H$ be f.g. groups, $\mathfrak{S}_{G}, \mathfrak{S}_{H}$ families of generating systems of $G, H$. If there is a bijection $\eta: G \rightarrow H$ such that

- for each $S_{G} \in \mathfrak{S}_{G}$ there is $S_{H} \in \mathfrak{S}_{H}$ which makes $\eta$ : Cay $\left(G, S_{G}\right) \rightarrow$ $\operatorname{Cay}\left(H, S_{H}\right)$ an isometry, and

- for each $S_{H} \in \mathfrak{S}_{H}$ there is $S_{G} \in \mathfrak{S}_{G}$ which makes $\eta^{-1}:$ Cay $\left(H, S_{H}\right) \rightarrow$ Cay $\left(G, S_{G}\right)$ an isometry.

Then $\operatorname{Isom}_{\mathfrak{S}_{G}}(G)$ and $\operatorname{Isom}_{\mathfrak{S}_{H}}(H)$ are isomorphic.

In particular, in the situations of Theorem 137. A, B, or D, or when $G$ and $H$ are both f.g. abelian without 2-torsion (case $(C)$ ), then $G$ and $H$ are isomorphic. 
Proof Define

$$
\begin{aligned}
\eta^{*}: \operatorname{Isom}_{\mathfrak{S}_{G}}(G) & \rightarrow \operatorname{Isom}_{\mathfrak{S}_{H}}(H) \\
\phi & \mapsto \eta \circ \phi \circ \eta^{-1}
\end{aligned}
$$

This is well-defined: For each $S_{H} \in \mathfrak{S}_{H}$ choose $S_{G} \in \mathfrak{S}_{G}$ such that $\eta$ is an isometry. Then $\eta \circ \phi \circ \eta^{-1}: H \rightarrow H$ is an isometry as well-vice versa for $\left(\eta^{*}\right)^{-1}:=\eta^{-1} \circ \cdot \circ \eta$. Hence, $\eta^{*}$ is a bijection, and, as one easily computes, indeed an isomorphism between groups.

In the cases (A), (B) and (D), we may directly conclude $G \cong H$. In the abelian case we just have $G \rtimes C_{2} \cong H \rtimes C_{2}$, but, as $G$ and $H$ are without 2-torsion, $G$ and $H$ must be isomorphic as well.

Example 143

Let us take a look at the three commensurable groups $G_{1}=\mathbb{Z}, G_{2}=\mathbb{Z} \rtimes C_{2} \cong$ $C_{2} * C_{2}$, and $G_{3}=\mathbb{Z} \times C_{2}$. For $G_{1}$, choose $S_{0}=\{ \pm 1, \pm 2\}$, and apply Theorem 137. $C$; for $G_{2}$ use Theorem 137. $D$ (c.f. previous example); for $G_{3}$ apply a direct calculation 1 . Then we find

$$
\begin{aligned}
& \operatorname{Isom}_{\mathfrak{S}}\left(G_{1}\right) \cong \mathbb{Z} \rtimes C_{2} \\
& \operatorname{Isom}_{\mathfrak{S}}\left(G_{2}\right) \cong \mathbb{Z} \rtimes C_{2} \\
& \operatorname{Isom}_{\mathfrak{S}}\left(G_{3}\right) \cong\left(\mathbb{Z} \rtimes C_{2}\right) \times C_{2} \cong G_{3} \rtimes C_{2} .
\end{aligned}
$$

We note that the resulting shared-isometry groups can be isomorphic, but might as well be just commensurable. And, as $G_{1}$ and $G_{2}$ are not isomorphic, we note that there cannot be a bijection $\eta: G_{1} \rightarrow G_{2}$ as in Lemma 142. The canonical inclusion $i: G_{1} \hookrightarrow G_{2}$ however might provide a deeper insight - it is a rough isometry for several generating systems.

\subsection{Shared Rough and Quasi-Isometries}

We have seen in Section 3.1 that it is sometimes possible to directly translate a proof into the rough context. This will be our goal for this section: To roughificate the proof of Theorem 137.

Definition 144

Let $G$ be a f.g. group. We call a family $\mathfrak{S}$ of finite generating systems of $G$ optimal if $\operatorname{URIsom}_{\mathfrak{S}}(G) \cong G$, and quasi-optimal if $\operatorname{UQIsom}_{\mathfrak{S}}(G) \cong G$.

\footnotetext{
${ }^{1}$ In this case it suffices to find the isometries for the standard generating set, the Cayley graph of which is a ladder. The cardinality of the second neighborhood of an edge in this graph depends on the order of its generating element, but must be preserved under isometries. This allows for a simple case distinction.
} 
Note that quasi-optimality is the stronger of both notions, as $\operatorname{URIsom~}_{\mathfrak{S}}(G) \subseteq$ $\mathrm{UQIsom}_{\mathfrak{S}}(G)$. Each translation from the left with an element of $G$ is a shared isometry, and hence we have

$$
G \leq \operatorname{Isom}_{\mathfrak{S}}(G) \subseteq \operatorname{URIsom}_{\mathfrak{S}}(G) \subseteq \operatorname{UQIsom}_{\mathfrak{S}}(G) .
$$

If $\mathfrak{S}$ is optimal, we also find $\operatorname{Iden}_{\mathfrak{S}}(G)$ to be trivial.

Lemma 145 (Optimality Lemma)

Let $G$ be a finitely generated group with $x^{y} \neq x^{-1}$ for all $x, y \in G$, unless $x=x^{-1}$. Let $G$ be non-abelian, or of exponent 2. Let $\mathfrak{S}$ be a family of finite generating systems of $G$ with the following Property 145:

- For each $g, h \in G$ with $g \neq h^{ \pm 1}$ and each $R \in \mathbb{N}^{*}$ there is $S=S(g, h, R) \in \mathfrak{S}$ such that $g \in S$ and $\|h\|_{S} \geq R$, or vice versa.

Then $\mathfrak{S}$ is quasi-optimal (and thus optimal).

Proof Let $\lambda, \epsilon \geq 0, \phi \in(\lambda, \epsilon)-\operatorname{Isom}_{\mathfrak{S}}(G)$, and $x, y \in G$ be arbitrary, let $z:=y^{-1} \cdot x$ and define

$$
z^{\prime}:=\phi(y)^{-1} \cdot \phi(x) \quad \Rightarrow \quad\left\|z^{\prime}\right\|_{S}=d_{S}(\phi(y), \phi(x)) .
$$

for all $S \in \mathfrak{S}$. Now assume $z^{\prime} \neq z^{ \pm 1}$. Then there is $S=S\left(z, z^{\prime},(1+\epsilon)\right.$. $(1+\lambda))$, such that $\phi$ is still a $(\lambda, \epsilon)$-quasi-isometry, and it holds

- either $z \in S$, then $\left\|z^{\prime}\right\|=d_{S}(\phi(y), \phi(x)) \leq \lambda d_{S}(y, x)+\epsilon=\lambda+\epsilon$, but $\left\|z^{\prime}\right\|_{S}>\lambda+\epsilon$ : contradiction,

- or $z^{\prime} \in S$, then $d_{S}(\phi(y), \phi(x))=1$, hence $d_{S}(y, x) \leq \lambda+\lambda \epsilon$, but $\|z\|_{S}>\lambda+\lambda \epsilon$ : contradiction!

Thus, $\phi(x)=\phi(y) \cdot\left(y^{-1} \cdot x\right)^{ \pm 1}$, or (after substitution): $\phi(y x)=\phi(y) \cdot x^{ \pm 1}$. The sign might still depend on $x$ and $y$, which we exclude in the next step.

Let $c:=\phi(e)$, and assume there are $x, y \in G$ with $\phi(x)=c x \neq c x^{-1}$, but $\phi(x y)=c(x y)^{-1} \neq c x y$. Then

$$
c \cdot(x y)^{-1}=\phi(x y)=\phi(x) y^{a}=c x y^{\alpha}
$$

for some $\alpha= \pm 1$, hence $x y^{\alpha}=y^{-1} x^{-1}$. If $\alpha=+1$, we have $(x y)^{2}=e$, and hence $\phi(x y)=c(x y)$. If $\alpha=-1$, we have $x^{y}=x^{-1}$, which contradicts our premise, unless $x=x^{-1}$. However, if $x=x^{-1}$, we have $\phi(x)=c x^{-1}$.

We conclude that $\phi(x)=c x$ for all $x \in G$, or $\phi(x)=c x^{-1}$ for all $x \in G$. The latter case leads to

$$
c y x=\phi\left(x^{-1} y^{-1}\right)=\phi\left(x^{-1}\right) y^{\beta}=c x y^{\beta}
$$

for all $x, y \in G$, and some $\beta= \pm 1$. Again, the case $\beta=-1$ leads to $y^{x}=y^{-1}$, which we excluded, unless $y=y^{-1}$. So both cases for $\beta$ lead to the conclusion 
that $G$ must be abelian. Indeed, in the abelian case, the inversion is a shared isometry of all symmetric finite generating systems, and it is non-trivial if and only if $G$ is not of exponent 2 .

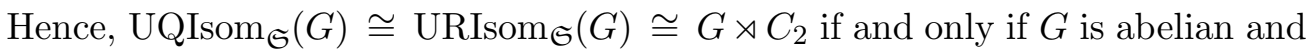
not of exponent 2 , $\operatorname{UQIsom}_{\mathfrak{S}}(G) \cong \operatorname{URIsom}_{\mathfrak{S}}(G) \cong G$ otherwise.

Example 146

146

No finite group has Property 145, as its diameter is limited. Torsion in itself is an obstruction to it: Let $G$ have Property 145, then each element of $G$ is either torsionsfree, or of exponent 1, 2, 3, 4, or 6 - these are those arguments for which the Euler totient function $\varphi$ is 2 or less ([S]]): Let $x \in G$ be an element with $x^{n}=e$. If $\varphi(n)>2$, we can choose two different generators $a$, $b$ of $C_{n}$, and hence $x^{a}$ and $x^{b}$ are powers of each other, and yet $\left(x^{a}\right) \neq\left(x^{b}\right)^{ \pm 1}$. Still, there might be other optimal or quasi-optimal families for these groups.

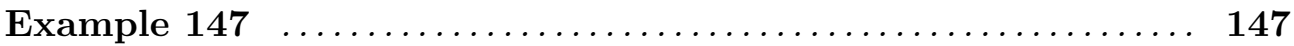

Let $F_{n}$ be the free group generated by $S_{0}$ with $\# S_{0}=n \geq 2$. Let $g, h \in F_{n}$, $g \neq h^{ \pm 1}$, and $R \in \mathbb{N}^{*}$ be arbitrary. Assume $h$ is not a power of $g$ and not neutral (otherwise switch them; both cannot happen as $F_{n}$ is torsionfree). If $g=e$, choose $x \in S_{0}$ such that $h$ is not a power of $x$, otherwise let $x=g$. Let $P$ be the maximum of $R$ and the wordlength of $h$ in $S_{0}$. Define

$S(g, h, R):=\{x\} \cup\left\{x^{(P+1)^{j}} s_{j} \mid s_{j} \in S_{0} \backslash\{x\}, j=1, \ldots \#\left(S_{0} \backslash\{x\}\right)\right\}$.

The exponents $(P+1)^{j}$ are chosen such that any non-trivial product of the elements $x^{(P+1)^{j}} s_{j}$ has large enough wordlength in $S_{0}$, that it cannot equal $h$, at least for the first $R$ steps in the Cayley graph. After this, the powers $x^{(P+1)^{j}}$ successively become available and "free" the generators $s_{j}$ to generate each other element of $F_{n}$, such that $\|h\|_{S} \geq R$. The family $\mathfrak{S}$ of all these generating systems is quasi-optimal due to Lemma 145.

Example 148

In a similar way, we may define quasi-optimal generating families for free abelian groups. We give the explicit example for $G=\mathbb{Z}$ (written additively): Again, assume $h$ is not neutral and not a multiple of $g$. If $g$ is zero, let $P=1+(R \vee|h|)$, otherwise choose $P \geq 1+(R \vee|h| \vee|g|)$ and coprime to $g$. Then define $S(g, h, R):=\left\{g, P^{2}, P^{3}+1\right\}$.

For arbitrary f.g. free abelian groups, do this componentwise.

The "delayed generation method" we applied in Examples 147 and 148 can sometimes be generalized to other f.g. groups: Choose a finite generating system $S_{0}$, then find a suitable element $x \in G$ such that $x$ and $g$ together do not generate $h$. Add $x^{P} s_{1}, x^{P^{2}} s_{2}, x^{P^{3}} s_{3}$ and so on, after choosing $P$ large enough and taking 
care for the group's relations: If e.g. holds $x^{P} s_{1}=s_{3}$, choose $P$ even larger, or change the sequence of the generators.

Question Is there a torsion-free group without Property 145, or which does not admit an optimal generating family?

Definition 149

Let $G$ and $H$ be f.g. groups, and let $\mathfrak{S}_{H}$ be a family of generating systems of $H$. We call a pair of maps $\eta: G \rightarrow H$ and $\eta^{\prime}: H \rightarrow G$ an $S_{H^{-}}$-semi-shared quasi-isometry if there are $\lambda, \epsilon \geq 0$ with:

- For each $S_{H} \in \mathfrak{S}_{H}$ there is a finite generating system $S_{G}$ of $G$ which makes $\left(\eta, \eta^{\prime}\right): \operatorname{Cay}\left(G, S_{G}\right) \rightarrow \operatorname{Cay}\left(H, S_{H}\right)$ a $(\lambda, \epsilon)$-quasi-isometry.

When we speak of an "S $\mathfrak{S}_{H}$-semi-shared quasi-isometry $\eta: G \rightarrow H$ " a suitable $\eta^{\prime}$ shall always be implied.

Theorem 150

Let $G$ and $H$ be f.g. groups with $x^{y} \neq x^{-1}$ for all $x, y \in G$ (resp. $H$ ), unless $x=x^{-1}$. Let $G$ and $H$ be non-abelian, or of exponent 2. Let $\mathfrak{S}_{G}$ and $\mathfrak{S}_{H}$ be quasi-optimal families of $G$ and $H$, respectively, and let $\eta: G \rightarrow H$ be an $\mathfrak{S}_{H^{-}}$ semi-shared quasi-isometry, such that $\eta^{\prime}$ is an $\mathfrak{S}_{G}$-semi-shared quasi-isometry. Then $G$ and $H$ are isomorphic as groups.

Proof We first note that $\eta \circ \eta^{\prime}: H \rightarrow H$ is an $\mathfrak{S}_{H^{-}}$-uniform quasi-isometry, and hence it is given by multiplication from the left with an element $c \in H$. Consider $\eta^{\prime \prime}: G \rightarrow H$ given by $h \mapsto c^{-1} \cdot \eta(h)$. Then $\left(\eta^{\prime \prime}, \eta^{\prime}\right)$ is another $\mathfrak{S}_{H^{-}}$ semi-shared quasi-isometry, $\left(\eta^{\prime}, \eta^{\prime \prime}\right)$ is a $S_{G^{-}}$-semi-shared quasi-isometry, and $\eta^{\prime \prime} \circ \eta^{\prime}$ is the identity.

Conversely, $\eta^{\prime} \circ \eta^{\prime \prime}: G \rightarrow G$ also is a multiplication from the left with an element $c^{\prime} \in G$. We easily find

$$
\begin{aligned}
\left(\eta^{\prime} \eta^{\prime \prime} \eta^{\prime} \eta^{\prime \prime}\right)(h) & =\left(c^{\prime}\right)^{2} \cdot h \\
& =\left(\eta^{\prime} \cdot \operatorname{id}_{H} \cdot \eta^{\prime \prime}\right)(h)=c^{\prime} \cdot h
\end{aligned}
$$

for all $h \in H$, thus $c^{\prime}=e$, and consequently $\eta^{\prime} \circ \eta^{\prime \prime}$ is the identity as well.

Without loss of generality, and to ease our notation, we may assume that $\left(\eta, \eta^{\prime}\right)$ already fulfills $\eta \circ \eta^{\prime}=\mathrm{id}_{H}$ and $\eta^{\prime} \circ \eta=\mathrm{id}_{G}$.

Now define

$$
\begin{aligned}
\xi: \operatorname{UQIsom}_{\mathfrak{S}_{H}}(H) & \rightarrow \operatorname{UQIsom}_{\mathfrak{S}_{G}}(G) \\
\phi & \mapsto \eta^{\prime} \circ \phi \circ \eta, \text { and } \\
\xi^{\prime}: \operatorname{UQIsom}_{\mathfrak{S}_{G}}(G) & \rightarrow \mathrm{UQIsom}_{\mathfrak{S}_{H}}(H) \\
\psi & \mapsto \eta \circ \psi \circ \eta^{\prime} .
\end{aligned}
$$


$\xi$ is well-defined: For each $S_{G} \in \mathfrak{S}_{G}$ choose $S_{H} \in \mathfrak{S}_{H}$ such that $\left(\eta, \eta^{\prime}\right)$ is a uniform quasi-isometry. Then for each $\phi \in \operatorname{UQIsom}_{\mathfrak{S}_{H}}(H)$, the map $\eta^{\prime} \circ \phi \circ$ $\eta: G \rightarrow G$ is a uniform quasi-isometry as well; the same accounts for $\xi^{\prime}$. Furthermore, we have

$$
\begin{aligned}
& \xi\left(\phi_{1}\right) \circ \xi\left(\phi_{2}\right)=\eta^{\prime} \phi_{1} \eta \eta^{\prime} \phi_{2} \eta=\xi\left(\phi_{1} \circ \phi_{2}\right) \\
& \xi^{\prime}\left(\psi_{1}\right) \circ \xi^{\prime}\left(\psi_{2}\right)=\eta \psi_{1} \eta^{\prime} \eta \psi_{2} \eta^{\prime}=\xi^{\prime}\left(\psi_{1} \circ \psi_{2}\right) \\
& \xi^{\prime}(\xi(\phi))=\eta \eta^{\prime} \phi \eta \eta^{\prime}=\phi \\
& \xi\left(\xi^{\prime}(\psi)\right)=\eta^{\prime} \eta \psi \eta^{\prime} \eta=\psi
\end{aligned}
$$

for all $g \in G$. This means that $\left(\xi, \xi^{\prime}\right)$ constitutes an isomorphism between $\operatorname{UQIsom}_{\mathfrak{S}_{H}}(H) \cong H$ and $\mathrm{UQIsom}_{\mathfrak{S}_{G}}(G) \cong G$.

A similar theorem should hold in the abelian case.

We now want to weaken the hypothesis of Theorem 150, with the goal of a sufficient criterion for commensurability. For this, we will rework the proof of Lemma 145] which yields a generalized form of homomorphism.

Lemma 151

Let $G$ and $H$ be f.g. groups, and $\mathfrak{S}_{H}$ a family of generating systems of $H$ satisfying Property 145. Let $\phi: G \rightarrow H$ be an $\mathfrak{S}_{H}$-semi-shared quasi-isometry with $\phi(e)=e$. Then $\phi$ fulfills $\phi(g h)=\phi(g) \cdot \phi(h)^{ \pm 1}$ for all $g, h \in G$, where the sign might depend on $g$ and $h$.

Proof Let $x, y \in G$ be arbitrary, $z=y^{-1} x$, and $z^{\prime}=\phi(y)^{-1} \phi(x)$. Assume $z^{\prime} \neq \phi(z)^{ \pm 1}$. Then we may choose $S_{H}=S\left(z^{\prime}, \phi(z),\left(\lambda^{2}+1\right) \cdot(\epsilon+1)\right) \in \mathfrak{S}_{H}$ a suitable generating system to separate $z^{\prime}$ from $\phi(z)$. Then we have

$$
\begin{aligned}
\left\|z^{\prime}\right\|_{S_{H}} & =d_{S_{H}}(\phi(y), \phi(x)) \\
& \leq \lambda \cdot d_{S_{G}}(y, x)+\epsilon=\lambda \cdot d_{S_{G}}(e, z)+\epsilon \\
& \leq \lambda^{2} \cdot d_{S_{H}}(\phi(e), \phi(z))+\lambda^{2} \epsilon+\epsilon \\
& =\lambda^{2} \cdot\|\phi(z)\|_{S_{H}}+\lambda^{2} \epsilon+\epsilon,
\end{aligned}
$$

and, similarly:

$$
\|\phi(z)\|_{S_{H}} \leq \lambda^{2} \cdot\left\|z^{\prime}\right\|_{S_{H}}+\lambda^{2} \epsilon+\epsilon
$$

Now one of $\left\|z^{\prime}\right\|_{S_{H}}$ and $\|\phi(z)\|_{S_{H}}$ is 1, while the other is larger than $\lambda^{2}+\lambda^{2} \epsilon+\epsilon$, contradiction. Hence, $z^{\prime}$ is $\phi(z)^{\alpha}$ for some suitable $\alpha= \pm 1$, which depends on $x$ and $y$. Substituting $y=g$ and $z=h$ yields $\phi(g h)=\phi(g) \cdot \phi(h)^{ \pm 1}$.

Note that it is always possible to switch from an arbitrary semi-shared quasiisometry $\phi$ to one with $\phi(e)=e$ by a simple translation. The translation even preserves the constants $\lambda$ and $\epsilon$ of the quasi-isometry. 
Theorem 152

Let $G, H$, and $\phi: G \rightarrow H$ be as in Lemma 151.

Assume one of the following statements holds:

1. G admits a generating system $S$ such that $(\phi(s))^{2}=e$ for each $s \in S$.

2. $G$ admits a generating system $S$ such that:

(a) There is no $x \in \phi\left(S \cup S^{-1}\right)$, with $x^{2}=e$.

(b) There are no $x, y \in \phi\left(S \cup S^{-1}\right), x \neq y^{ \pm 1}$, with $x^{2}=y^{2}$.

(c) There are no $x, y \in \phi\left(S \cup S^{-1}\right), x \neq y^{ \pm 1}$, with $(x y)^{2}=e$.

(d) There are no $x, y \in \phi\left(S \cup S^{-1}\right), x \neq y^{ \pm 1}$, with $x^{y}=x$.

(e) There are no $x, y \in \phi\left(S \cup S^{-1}\right), x \neq y^{ \pm 1}$, with $x^{y}=x^{-1}$.

(f) There are at least two distinct elements in $S$, which are not inverses of each other.

(In particular, $G$ is not abelian.)

Then $G$ and $H$ are commensurable up to finite kernels (Definition [123).

Proof Due to Lemma 151 we have in each case

$$
\phi(g h)=\phi(g) \cdot \phi(h)^{\sigma(g, h)}
$$

with $\sigma(g, h) \in\{ \pm 1\}$ for any $g \in G$ and $h \in H$. Observe that $\sigma(g, e)=\sigma(e, g)$ $=+1$. If $\phi(h)$ is neutral or of order 2, we choose $\sigma(g, h)$ to be +1 without loss of generality. We next show that under both hypothesis $\phi$ must be a homomorphism. Due to Proposition $125 G$ and $H$ then must be commensurable up to finite kernels.

(1) We trivially have

$$
\phi(g s)=\phi(g) \cdot \phi(s)
$$

for any $g \in G$ and $s \in S$. By induction, $\phi$ must be a homomorphism.

(2) Let $g \in G$ and $s, t \in S$ be arbitrary, $s \neq t^{ \pm 1}$. We make use of the associative law:

$$
\begin{aligned}
\phi(g s t) & =\phi(g) \cdot \phi(s)^{\alpha} \cdot \phi(t)^{\beta} \\
& =\phi(g) \cdot\left(\phi(s) \cdot \phi(t)^{\gamma}\right)^{\delta}
\end{aligned}
$$

for some $\alpha, \beta, \gamma, \delta= \pm 1$. The sixteen possible cases resolve as in Table 3.1 Fourteen cases subsequently contradict our premise. Both remaining cases 1 and 7 demand $\alpha=\sigma(g, s)=+1$, for all $g \in G$ and $s \in S$, so we have

$$
\phi(g s)=\phi(g) \cdot \phi(s),
$$

and, again by induction, $\phi$ must be a homomorphism. 


\begin{tabular}{|r|l|l|l|l|l|l|}
\hline $\mathrm{Nr}$ & $\alpha$ & $\beta$ & $\gamma$ & $\delta$ & $x^{\alpha} \cdot y^{\beta}=\left(x \cdot y^{\gamma}\right)^{\delta}$ & contradiction \\
\hline 1 & + & + & + & + & - & no \\
2 & + & + & + & - & $(x y)^{2}=e$ & yes (c) \\
3 & + & + & - & + & $y^{2}=e$ & yes (a) \\
4 & + & + & - & - & $x^{y}=x^{-1}$ & yes (e) \\
5 & + & - & + & + & $y^{2}=e$ & yes (a) \\
6 & + & - & + & - & $x^{y}=x^{-1}$ & yes (e) \\
7 & + & - & - & + & - & no \\
8 & + & - & - & - & $\left(x y^{-1}\right)^{2}=e$ & yes (c) \\
9 & - & + & + & + & $x^{2}=e$ & yes (a) \\
10 & - & + & + & - & $y^{x}=y^{-1}$ & yes (e) \\
11 & - & + & - & + & $x^{2}=y^{2}$ & yes (b) \\
12 & - & + & - & - & $x^{y}=x$ & yes (d) \\
13 & - & - & + & + & $x^{-2}=y^{2}$ & yes (b) \\
14 & - & - & + & - & $x^{y}=x$ & yes (d) \\
15 & - & - & - & + & $x^{2}=e$ & yes (a) \\
16 & - & - & - & - & $y^{x}=y^{-1}$ & yes (e) \\
\hline
\end{tabular}

Table 3.1: The sixteen cases of the proof of Theorem [152]3. For convenience, we use $x=\phi(s)$ and $y=\phi(t)$.

Corollary 153

Let $G, H$, and $\phi$ be as in Theorem 152, and let $H$ be non-abelian, or of exponent 2. In addition, $x^{y} \neq x^{-1}$ shall hold for all $x, y \in H$ with $x \neq x^{-1}$. Then $H$ is the quotient of $G$ by the finite subgroup $\operatorname{ker} \phi \unlhd G$.

Proof Lemma 145] ensures that $\phi \circ \phi^{\prime}: H \rightarrow H$ is given by multiplication with a fixed element of $H$, and in particular, $\phi$ must be surjective. From the proof of Theorem 152 we know that $\phi$ is a homomorphism with finite kernel. Using the First Isomorphism Theorem (Bo], Korollar 1.2.7), we see $H=\operatorname{im} \phi \cong G / \operatorname{ker} \phi$.

Unfortunately, Proposition 134 is not yet strong enough to constitute a reversal of Corollary 153. Still, we are confident to soon find a sustainable connection between semi-shared quasi-isometries and quotients of finite kernel. Through the means of residual finiteness, it might then be possible to finally find a perfectly fitting geometrical equivalence relation which equals commensurability-which was our motivation for this chapter. 


\section{Bibliography}

[AP] N. Aronszajn, P. Panitchpakdi, Extension of Uniformly Continuous Transformations and Hyperconvex Metric Spaces, Pacific J. Math. 6, (1956) 405-439, http://projecteuclid.org/handle/euclid.pjm/1103043960

[Ba] R. Baer, Endlichkeitskriterien für Kommutatorgruppen, Math. Annalen 124 (1952) 161-177

[BBI] D. Burago, Y. Burago, S. Ivanov, A Course in Metric Geometry, Graduate Studies in Mathematics Volume 33, AMS

[Bi1] G. Birkhoff, Lattice Theory, American Mathematical Society Colloquium Publications Vol. XXV, 2nd ed. (1948) and 3rd ed. (1960)

[Bi2] G. Birkhoff, Von Neumann and Lattice Theory, Bull. Amer. Math. Soc. 64, Nr 3, Part 2 (1958) 50-56, http://www.ams.org/bull/1958-64-03/S0002-9904-1958-10192-5/ S0002-9904-1958-10192-5.pdf

[Bo] S. Bosch, Algebra, 4th edition, Springer-Verlag (2001)

[dH] P. de la Harpe, Topics in Geometric Group Theory, The University of Chicago Press (2000)

[EK] R. Espínola, M. A. Khamsi, Introduction to Hyperconvex Spaces, appeared in Handbook of Metric Fixed Point Theory by W. A. Kirk and B. Sims, Kluwer 2001, http://drkhamsi.com/publication/Es-Kh.pdf

[FM] B. Farb, L. Mosher, On the asymptotic geometry of abelian-by-cyclic groups, Acta Mathematica 184, 2 (2000) 145-202, preprint available at http://arxiv.org/abs/math.GR/0005181

[GKM] T. Gelander, A. Karlsson, G. A. Margulis, Superrigidity, Generalized Harmonic Maps and Uniformly Convex Spaces, Geom. funct. anal. 17 (2008) 1524-1550, preprint at http://arxiv.org/abs/math/0606256

[Gl] V. Glivenko, Géometrie des systèmes de chosen normées, Am. Jour. of Math. 58 (1936) 799-828

[Gr] G. A. Grätzer, General Lattice Theory, 2nd ed., Birkhäuser Verlag (2003) 
[Gv1] M. Gromov, Hyperbolic manifolds, groups and actions, Ann. of Math. Studies 97, Princeton Univ.Press, Princeton (1981) 183-213

[Gv2] M. Gromov, Metric Structures for Riemannian and Non-Riemannian Spaces, Progress in Mathematics 152, Birkhäuser (1999)

[Gv3] M. Gromov, Asymptotic Invariants of Infinite Groups in Geometric Group Theory, Volume 2, LMS Lecture Notes Series 182, Cambridge University Press

[He] J. Heinonen, Lectures on Analysis on Metric Spaces, Springer NY (2001)

[Ho] H. S. Holdgrün, Analysis, Band 2, Leins Verlag Göttingen (2001)

[HR] D. H. Hyers, T. M. Rassias, Approximate homomorphisms, Aequationes Math. 44 (1992) 125-153, http://wWw .digizeitschriften.de/index.php?id=166\&ID=379571

[HU] D. H. Hyers, S. M. Ulam, Approximate Isometries of the Space of Continuous Functions, Ann. of Math. 48 (2) (1947) 285-289

[Hy] D. H. Hyers, On the Stability of the Linear Functional Equation, Proc. Nat. Acad. Sci. USA, 27 (1941) 222-224, http://www .pnas.org/content/27/4/222.full.pdf+html

[I] J. R. Isbell, Six theorems about injective metric spaces, Commentarii mathematici Helevetici 39 (1964) 65-76, http://wWw.digizeitschriften.de/resolveppn/GDZPPN002058340

[Kn] M. Kanai, Rough Isometries, and combinatorial approximations of geometries of non-compact riemannian manifolds, J. Math. Soc. Japan 37 (1985) 391-413

[Kp] I. Kaplansky, Lattices of continuous functions, Bull. Am. Math. Soc. 53 (1947) 617-622

[L] C. H. Li, On isomorphisms of finite Cayley graphs - a survey, Discrete Mathematics 256 (2002) 301-334

[Me] K. Menger, Untersuchungen über allgemeine Metrik, Mathematische Annalen 100 (1928) 75-163, http://www.digizeitschriften.de/resolveppn/PPN235181684_0100

[Mn] B. Monjardet, Metrics on partially ordered sets - a survey, Discrete Mathematics 35 (1981) 173-184

[Mo] G. D. Mostow, Strong rigidity of locally symmetric spaces, Ann. of Math. Studies 78, Princeton Univ.Press, Princeton (1973) 183-213

[MU] S. Mazur, S. Ulam, Sur les transformations isométriques d'espaces vectoriels normés, C. R. Acad. Sci., Paris 194 (1932), 946-948 
[P] P. Petersen V, Gromov-Hausdorff Convergence of Metric Spaces, AMS Proc. Symp. in Pure Math Math. 54 (3) (1993) 489-504

[Ra1] T. M. Rassias, Isometries and approximate isometries, Internat. J. Math. and Math. Sciences, 25 (2) (2001) 73-91,

http://www.hindawi.com/GetArticle.aspx?doi=10.1155/

\section{S0161171201004392}

[Ra2] T. M. Rassias, A New Generalization of a Theorem of Jung for the Orthogonality Equation, Applicable Analysis, 81 (2002) 163-177, and: V. A. Fă̌ziev, T. M. Rassias, P. K. Sahoo, The Space of $(\psi, \gamma)$-additive Mappings on Semigroups, Trans. AMS, 354 Number 11 (2002) 44554472, http://www.ams.org/tran/2002-354-11/ S0002-9947-02-03036-2/S0002-9947-02-03036-2.pdf

[Re] M. Requardt, Continuum Limit of Discrete Geometries, Int. J. Geom. Meth. Mod. Phys. 3 (2006) 285

[Ro] R. T. Rockafellar, Convex Analysis, Princeton University Press (1970)

[S] N. J. A. Sloane, On-Line Encyclopedia of Integer Sequences, sequence A000010, http://www.research.att.com/ njas/sequences/A000010

[T] A. M. Turing, On Computable Numbers, with an Application to the Entscheidungsproblem, Proc. London Math. Soc. Ser. 2, 42 (1937) 230265

[U] S. M. Ulam, A Collection of Mathematical Problems, Interscience Publ., New York (1960)

[V] J. Väisälä, A proof of the Mazur-Ulam theorem, Amer. Math. Mon. 110, 7 (2003) 633-635, http://www.helsinki.fi/ jvaisala/mazurulam.pdf

[vN] J. von Neumann, Lectures on continuous geometries, Princeton 19361937 (2 vols.), in particular chapter XVII

[Wr] D. Werner, Funktionalanalysis, Springer (2005)

[Wv] N. Weaver, Lipschitz Algebras, World Scientific (1999) 


\title{
curriculum vitæ
}

\section{Dipl.-Phys. Andreas Lochmann}

Geboren am

Familienstand

Staatsbürgerschaft

Adresse

eMail
4.1.1980 in Bad Pyrmont, Niedersachsen

ledig

deutsch

Merkurstr. 31b

93051 Regensburg

and.lochmann@googlemail.com

\section{Schulischer und nachschulischer Werdegang}

\author{
$1986-1990$ \\ $1990-1999$ \\ St. Marien-Grundschule Lügde \\ Städtisches Gymnasium Blomberg \\ Mai 1999 \\ (heutiges Hermann-Vöchting-Gymnasium) \\ Abitur (1,1) \\ Mai 1999 - Mai 2000 \\ Zivildienst in der Kurklinik Friedrichshöhe \\ in Bad Pyrmont
}

\section{Studium}

ab September 2000

Studium der Physik und Mathematik an der Georg-August-Universität Göttingen, Stipendium durch die Studienstiftung des deutschen Volkes

5. Juli 2002

Vordiplom Physik ('Sehr gut')

25. April 2003

Vordiplom Mathematik ('Sehr gut')

11. November 2005

Diplom Physik ('Sehr gut') unter Anleitung von PD Dr. Manfred Requardt

ab Dezember 2005

Promotionsstudiengang in Mathematik an der Georg-August-Universität Göttingen, Stipendium durch das Graduiertenkolleg «Gruppen und Geometrie»

\section{Konferenzen und Auslandsaufenthalte}

August 2001

August 2002

August 2006

Oktober 2006

Juni 2007

Dezember 2007

Mai 2008

August 2008

November 2008
Sommerakademie der Studienstiftung zum Thema

"Physik der Fluide», La Villa, Italien

Sommerakademie der Studienstiftung zum Thema

«Künstliche Intelligenz», Ftan, Schweiz

"Young Topologists and New Topology», Bedlewo, Polen «Göttingen Conference on Coarse Geometry '06», Göttingen «Joint International Meeting UMI-DMV», Perugia, Italien Winter School «Geometric Group Theory», Göttingen "Young Topologists' Meeting 2008», Le Châtelard, Schweiz Workshop «Groups and Infinite Graphs», Wien, Österreich Autumn School «Geometric Invariants of Groups», Göttingen 


\section{Mitarbeit in der Fakultät}

Oktober 2006

WS 2006/2007

Dezember 2007

$2007-2008$

WS 2008/2009
Aufbau der Internetpräsenz der «Göttingen Conference on Coarse Geometry '06»

Wissenschaftliche Hilfskraft für «Differential- und Integralrechnung I» unter Prof. Dr. Thomas Schick

Aufbau der Internetpräsenz des Graduiertenkollegs «Mathematische Strukturen in der modernen Quantenphysik»

Studentischer Sprecher im Graduiertenkolleg «Gruppen und Geometrie»

Fachliche Betreuung der Bachelor-Arbeit «Durchmesser und Wachstum endlicher Heisenberggruppen» von Patrick Neumann

\section{Wissenschaftliche Veröffentlichungen}

September 2004

Oktober 2007
«An Analysis of the Transition Zone between the various Scaling Regimes in the Small-World Model» in Kooperation mit PD Dr. Manfred Requardt, erschienen 2006 im Journal of Statistical Mechanics Preprint «Rough Isometries of Lipschitz Function Spaces»

\section{Weitere Betätigungen}

Juli 1999

seit 2005

Dezember 2008

seit Oktober 2009
Teilnahme an der 30. Internationalen Physik Olympiade in Padua, Italien ('Silber') Mitarbeiter am Opensource-Projekt «Enigma» Stimme und akustische Nachbearbeitung für den mathematischen Lehrfilm «Dimensions» Übersetzung des Buches "Analysis by its History» von E. Hairer und G. Wanner ins Deutsche. 